\title{
10. Microscopy of defects in semiconductors
}

\author{
F. C.-P. Massabuau, J. Bruckbauer, C. Trager-Cowan, R.A. Oliver
}

\subsection{Introduction}

In the study of defects in semiconductors, many techniques provide access to the structure or properties of ensembles of defects. Microscopy techniques, however, allow us to access the structure and properties of individual defects. Whilst in one sense this can be a weakness, leading to results which lack statistical significance, the ability to image defects at high resolution can provide a clarity of understanding and a wealth of information which is extraordinarily valuable. It is said that "seeing is believing", and microscopy represents the single most direct method to "see" defects, or rather to image them, since the resolution of modern microscopes extends right down to the atomic scale, far beyond the power of human sight. Increasingly, microscopy techniques also provide access to nanoscale resolution of the properties of defects and material in their close vicinity, allowing direct correlation of structure and properties.

There are any number of microscopy techniques that can be useful in addressing defects in semiconductors, and here we choose to focus on three main families: scanning probe microscopy (SPM), scanning electron microscopy (SEM) and transmission electron microscopy (TEM). We note that other powerful microscopy techniques, particularly atom probe tomography (APT) are addressed elsewhere in this book. In this chapter, we will first address the basic principles of our selected microscopy techniques (Section 10.2): it is vital that the reader who either chooses to use microscopy in their own research on defects or relies on microscopy data from the literature, should understand the physical principles underlying image formation and hence the strengths and (perhaps more importantly) limitations of the techniques. In our discussions of image formation, we will elucidate the mechanisms by which defects are typically imaged in each technique. Then, in the latter part of the chapter (Section 10.3), we describe some key examples of the application of microscopy to semiconductor materials, addressing both point and extended defects and both 2D and 3D materials.

\subsection{Basic principles of the microscopy techniques}

\subsubsection{Scanning probe microscopy \\ 10.2.1.1 Atomic force microscopy}

In its most basic form, the atomic force microscope (AFM) is a tool for measuring surface morphology at the nanoscale. Hence, where defects intersect a surface and affect the surface topography, AFM can be used to identify them. Whilst it is possible to achieve atomic resolution imaging in AFM in ultrahigh vacuum, for semiconductors this is more usually achieved using scanning tunnelling microscopy (STM) which is described in Section 10.2.1.3. Here, we will address the application of AFM in air to image nanoscale (but not atomic level) surface features, related to defects. Examples of defects which influence the surface topography include dislocations, which necessarily create a pit where they intersect the surface, in order that the surface tension may balance the dislocation line tension. Dislocation imaging in AFM is discussed is one of the examples we address later in the chapter, in Section 10.3.1.2. Here we address the principles of AFM and the basic image formation mechanism [1].

AFM employs a sharp tip, with end radius typically less than $10 \mathrm{~nm}$, suspended from the end of a microcantilever. In the simplest realisation of the technique, a piezo-electric scanner is used to scan the tip over the surface in contact with the sample, and where it encounters a change in the surface topography, the cantilever deflects. This deflection is monitored by reflecting a laser beam from the back side of the cantilever onto a photo-detector. If the path length followed by the laser beam is long, nanoscale motion of the cantilever is amplified into larger changes in the position of the laser beam at the photodetector, and hence the surface morphology can be measured. This very simple implementation of AFM, similar to the operation of a record player, is rarely used, and indeed even when the technique was first invented [2], a feedback circuit was employed to control the deflection of the cantilever and hence the force on the surface. The feedback circuit acts to continuously adjust the height of the cantilever, aiming to achieve a fixed cantilever (or equivalently laser spot) deflection. This 
fixed deflection value is the "setpoint" for the feedback circuit. The cantilever height is adjusted by changing the voltage applied to the z-piezo - a piezoactuator oriented to provide motion in the z-direction (i.e. normal to the surface). These voltages are then converted to heights using calibration data. The accuracy of an AFM is hence only as good as the calibration data it relies on, and the calibration should usually be checked approximately every six months. The instrumentation used in AFM is illustrated schematically in Figure 1. Note, that the figure shows a system in which the tip is scanned and the sample held stationary. This configuration allows large samples (such as full semiconductor wafers) to be scanned. However, many microscopes, particularly those used for smaller samples keep the tip stationary and achieve relative motion between the tip and sample by moving the sample using a piezoactuator.

The vertical resolution of a well-calibrated AFM is limited in theory by the ability to control the zmotion via the application of voltage to the z-piezo. In practice, the vertical resolution is actually determined by noise, and the noise floor of typical commercial microscopes is often less than $0.5 \AA$, allowing the imaging of atomic-scale features such as monolayer steps. The horizontal resolution is in general determined by the radius of the apex of the tip used for imaging. Since the forces acting on the tip include fairly long-range van der Waal's interactions, even where portions of the tip away from the very apex do not directly contact the surface they still influence the tip deflection, and hence the resolution. Hence, the creation and preservation of a sharp tip is paramount if high resolution imaging is to be achieved.

When an AFM is operated, as described above, with the tip in constant contact with the surface, this is known as "contact mode". The constant rubbing of the tip over the surface tends to wear the tip, blunting its sharp apex (or sometimes damaging the sample) so many users prefer to employ an "intermittent contact" mode instead to reduce tip wear and hence improve resolution. The most common intermittent contact mode is the so-called "tapping mode". In tapping mode the tip is oscillated at a frequency close to its resonant frequency, usually by a small piezo-element on the tip holder. When the tip is close enough to the surface to make contact at the bottom of each oscillation,

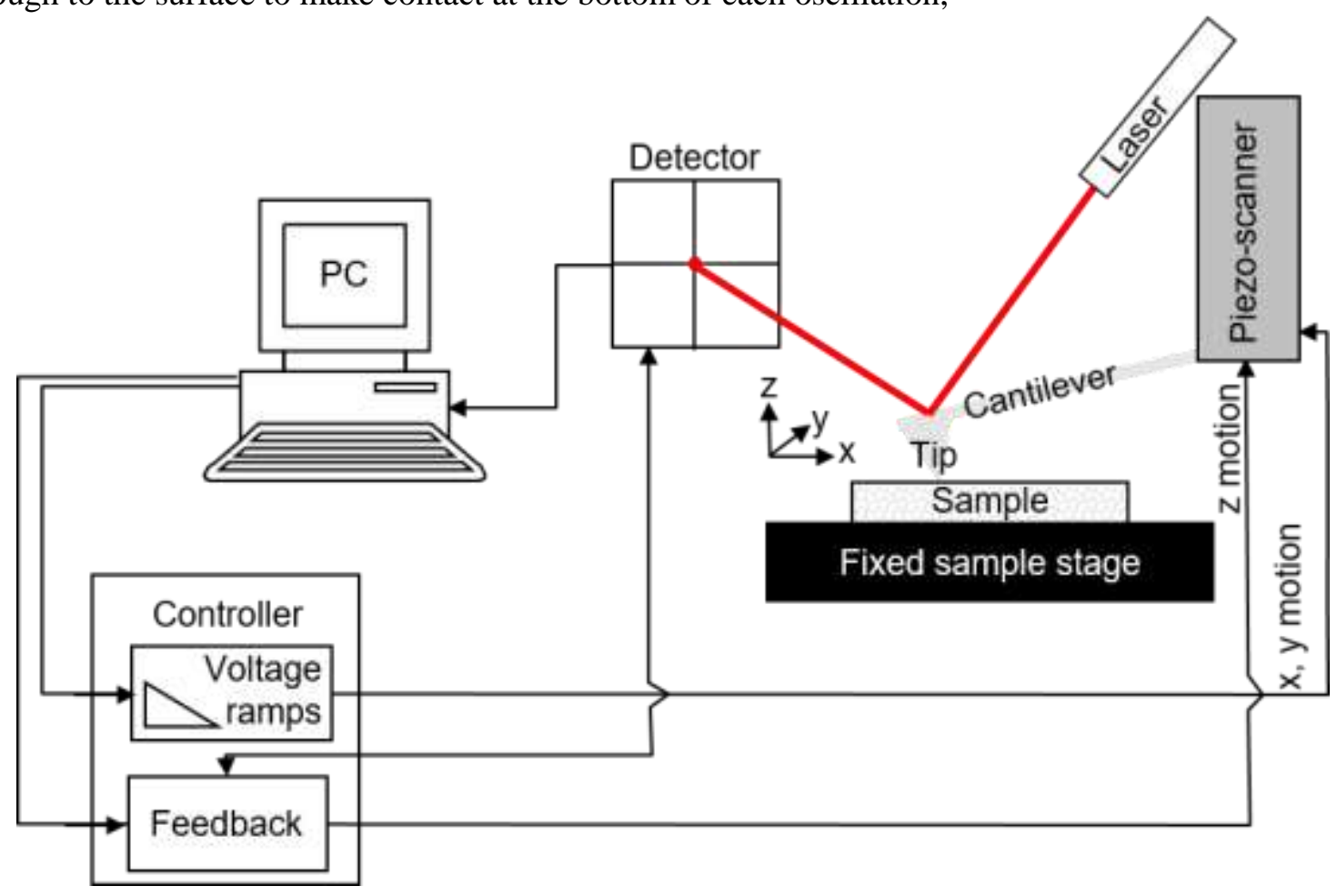

Figure 1: Schematic illustration of an atomic force microscope for a tip-scanning system

the presence of the surface limits or damps the amplitude of oscillation, which varies linearly with tipsample separation. Hence, the amplitude of oscillation may be used as the input parameter to a feedback circuit: a constant amplitude setpoint is maintained by varying the voltage applied to the z-piezo, so that the tip traces out a contour of constant surface height. As in contact mode, calibration data is used to convert the voltages applied to the z-piezo to heights, forming the topography imaging. The amplitude 
of vibration is also affected by a second mechanism even when the tip does not contact the surface: the natural frequency of cantilever oscillation is a function of the force gradient experienced by the cantilever. Since the frequency of the driving oscillation is fixed, changes in the natural frequency can take the vibration on or off resonance, changing the amplitude of vibration. This effect has less influence on tapping mode imaging than the damping of the amplitude, but is used in "non-contact" mode to image the surface whilst applying minimal forces to the tip and sample.

Non-contact imaging is not usually an effective way to achieve high resolutions in air, since most samples imaged in air are covered by a layer of fluid from atmospheric humidity, and non-contact imaging requires the tip to remain beyond this fluid layer, which tends to blur the outlines of surface features. To achieve higher lateral resolutions in air, alternative approaches have recently been developed with the aim of controlling and minimising the force on the tip to maintain its sharpness. An example of this is "PeakForce" tapping mode [3], in which rather than oscillating the tip close to resonance, the tip is oscillated off resonance by the main z-piezo and its deflection is measured throughout each oscillation cycle. The variation in force during the oscillation cycle is then estimated, and the highest force experienced during the period in which the tip is in contact with the surface is monitored. This is the "peak force" and is used as the feedback parameter, so that the cantilever traces a contour over the surface at which this peak force is constant. There is increasing evidence that by maintaining low peak forces throughout imaging, blunting of the tip is minimised, facilitating high lateral resolutions in imaging, but the influence of materials properties, rather than topography, on this mode are not yet fully understood.

\subsubsection{Electrical characterisation techniques in AFM}

The potential uses of the AFM tip extend far beyond topographic imaging, with many opportunities available to measure the properties of materials. Characterisation of electrical properties generally requires a conductive tip, and this can be achieved by coating a standard silicon tip in a metallic layer. It is important that the metallic layer is robust enough to avoid it wearing away during imaging, and PtIr layers are often used for this purpose. An alternative is to use all-metallic pyramid structures on the end of metal or silicon cantilevers, but whilst such structures overcome problems with coating wear, these tips are more likely to deform plastically, becoming blunt. For some applications, particularly scanning spreading resistance microscopy (SSRM) of Si, tips coated with or fully formed from highly-doped diamond are used, providing a hard, mechanically stable imaging probe.

SSRM is part of a family of techniques which also includes conductive AFM (C-AFM) and tunneling AFM (TUNA). In these techniques, the tip is usually kept in direct contact with the sample surface, a bias is applied between the tip and the sample, and the resulting current flow is monitored. The techniques differ mainly in the nature and sensitivity of the amplifiers used to measure the current, with SSRM employing a logarithmic amplifier to access the resistivity of a broad range of samples, whilst C-AFM and TUNA employ linear amplifiers, with TUNA addressing smaller currents than C-AFM. Very recently, PeakForce TUNA [4] and other similar techniques have allowed currents to be measured in intermittent contact modes during the contact phase of the oscillation cycle, allowing current maps of very fragile materials to be built up.

These current flow techniques are applicable to any material with sufficient conductivity to carry a measureable current. More specific to semiconductors is scanning capacitance microscopy (SCM). SCM is again a contact mode technique. Assuming that in air a semiconductor surface has a native oxide present, the metal coating on the surface, the native oxide and the semiconductor itself form a metal-oxide-semiconductor (MOS) capacitor (see Figure 2(a)). The capacitance of such a device varies with applied voltage in a way that is dependent on the local carrier concentration. Consider the application of a negative bias to a tip in contact with an n-type semiconductor. The electrons are repelled from the tip, effectively increasing the thickness of insulating material between the metallic tip and the underlying semiconductor, and the capacitance of the device is reduced. If the device were left to equilibrate, minority carriers would accumulate at the semiconductor oxide interface, and the capacitance would then be controlled just by the oxide thickness. However, if the bias applied to the tip varies at high frequency there is insufficient time for this accumulation to occur. (The relevant $\mathrm{C}-\mathrm{V}$ curves are illustrated schematically in Figure 2(b)). Under these circumstances, the amplitude of the change in capacitance with applied voltage is a function of the carrier concentration in the semiconductor, and hence on the dopant concentration, because for the same applied change in voltage a larger depletion region forms in a lightly doped semiconductor than a highly doped semiconductor, causing a larger change in capacitance. (The variation in the $\mathrm{C}-\mathrm{V}$ curves with dopant concentration is 
illustrated schematically in Figure 2(c)). The rate of change of capacitance with voltage is monitored by a Gigahertz frequency capacitance sensor and is used as a measure of the carrier density in the material. The phase of the capacitance response relative to the drive voltage provides information as to whether depletion occurs during the positive or negative half of the drive cycle, hence determining whether the material is doped n- or $\mathrm{p}$-type.

A typical SCM data set thus consists of two images; (1) A phase image in which n-type material is seen as black and p-type as white. Non-semiconducting material (insulating or metallic) does not deplete and

(a)

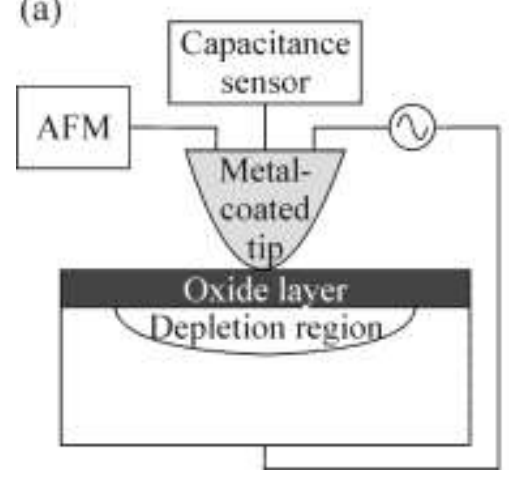

(b)

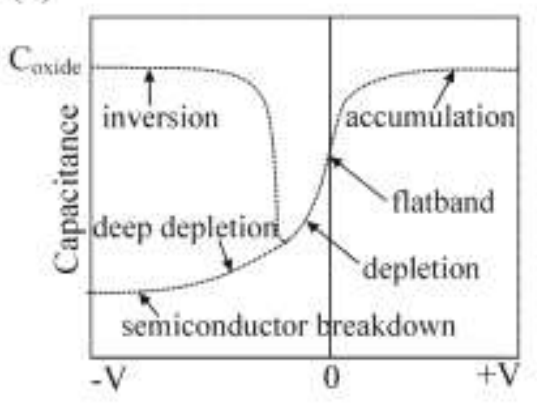

(c)

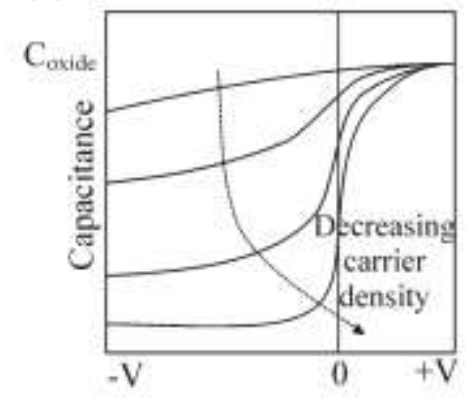

Figure 2: Schematic illustrations of (a) the instrumentation for SCM, (b) C-V curves for an n-type semiconductor for lowfrequency bias changes (leading to inversion) and high frequency bias changes (leading to deep depletion) and ( $b$ ) $C$ - $V$ curves for n-type semiconductors with different carrier densities for high frequency bias changes. $C_{\text {oxide }}$ is the capacitance of the oxide layer at the semiconductor surface.

no fixed phase can be measured, so such regions appear grey and noisy. (2) An amplitude image in which highly doped material is seen as having a lower amplitude than lightly doped material, typically represented by a lower brightness in a greyscale image. It should however be noted that undoped (or non-semiconducting) material shows no change in capacitance with voltage and thus yields no signal (seen as an even lower brightness in a greyscale image).

SCM and SSRM are very widely used in the study of semiconductors. Perhaps slightly less common is electrical force microscopy, and its more quantitative variant Kelvin probe force microscopy (KPFM). These techniques usually employ a lift mode - in which the topography of the sample is recorded during an initial scan line, and then the tip is raised through a fixed height and rescans the same trajectory during a second scan line during which electrical property data is collected. The advantage of this approach is that during the topography scan the tip's oscillation is strongly affected by forces arising from contact between tip and the sample. In the lift scan however, forces arising from the potential difference between a biased tip and the sample may be more easily detected, allowing measurement of the variation in potential across the sample surface.

As with topography, all electrical AFM techniques have resolution limited by the finite size of the tip. Furthermore, the sample topography can profoundly influence the electrical data, since changes in the tip-sample interaction area as the tip passes over varying topographic features alter the electrical interaction. C-AFM provides a simple example: if the tip-sidewall runs up against a three-dimensional (3D) island forming a large contact area, an increased current can flow, even though the properties of the material may be unchanged relative to an adjoining area. Similar issues apply to the other techniques mentioned here, and to myriad other methods which have been developed to probe electrical materials in the AFM. This short section does not provide an exhaustive account, but rather a flavour of the available options. For further details the reader should consult reference [5]. One interesting recent development worthy of mention is the integration of light sources with AFM setups to allow measurement either in darkness or with illumination. This approach is most commonly used to assess the photoconductivity of solar cell materials in C-AFM or TUNA [6], but is equally applicable to other electrical AFM techniques and provides a wealth of new opportunities for the characterization of optoelectronic materials.

\subsubsection{Scanning tunneling microscopy}

The most common application of scanning tunneling microscopy (STM) in the study of semiconductors is in the study of the atomic structure of clean (i.e free from native oxide) semiconductor surfaces in ultra-high vacuum. In this context, the termination of extended defects at the surface may be observed 
in exquisite detail [7]. Point defects in the surface structure are frequently observed and, whilst defects seen following surface cleaning or material deposition may not be representative of bulk defects, insight into defects in the bulk may be achieved by careful interpretation of images of cleaved surfaces [8]. Specially prepared samples are sometimes required, for example material grown at lower temperatures, to ensure a sufficient density of defects to allow the relevant features to be found and identified. Where shallow sub-surface point defects influence the electronic structure of the overlying surface layers, they may be detected, in addition to defects immediately at the surface. In two dimensional (2D) materials the distinction between surface and bulk defects is essentially meaningless, since there is no 3D bulk material. Hence STM may find increasing application in assessing point defects in such novel structures. Unlike AFM, STM does not utilize a deflecting cantilever. Instead a very sharp metallic needle forms the tip and is scanned over the sample surface, in very close proximity but with a few Angstrom vacuum gap between the two. Under the influence of an applied bias, electrons may tunnel across the gap, but the magnitude of the tunneling current is exponentially dependent on the distance between the tip and the sample. Hence, the contribution of atoms away from the very apex of the tip to imaging is much smaller than in AFM, and it is this exponential dependence that allows STM to be used routinely for atomic resolution imaging, with the tunneling current flowing predominately between a single atom at the sample surface and a single atom at the tip apex. Similar to AFM, a feedback circuit may be employed, with a z-piezo being used to maintain a fixed tunneling current. The voltages applied to the z-piezo form a map of the topography of the sample, with the tip tracing out a contour of constant tunneling current, equivalent to a constant tip-sample distance. This simple description immediately begs the question: what do we mean by a constant tip-sample distance in this situation? Simple models of the tip-sample interaction indicate that a better description of an atomic resolution STM image at constant tunneling current is a contour of constant local density of states (LDOS) at the Fermi level of the sample surface [9]. Whilst the original models from which this understanding is derived are known to be incomplete, they provide a useful starting point for discussion of STM data,
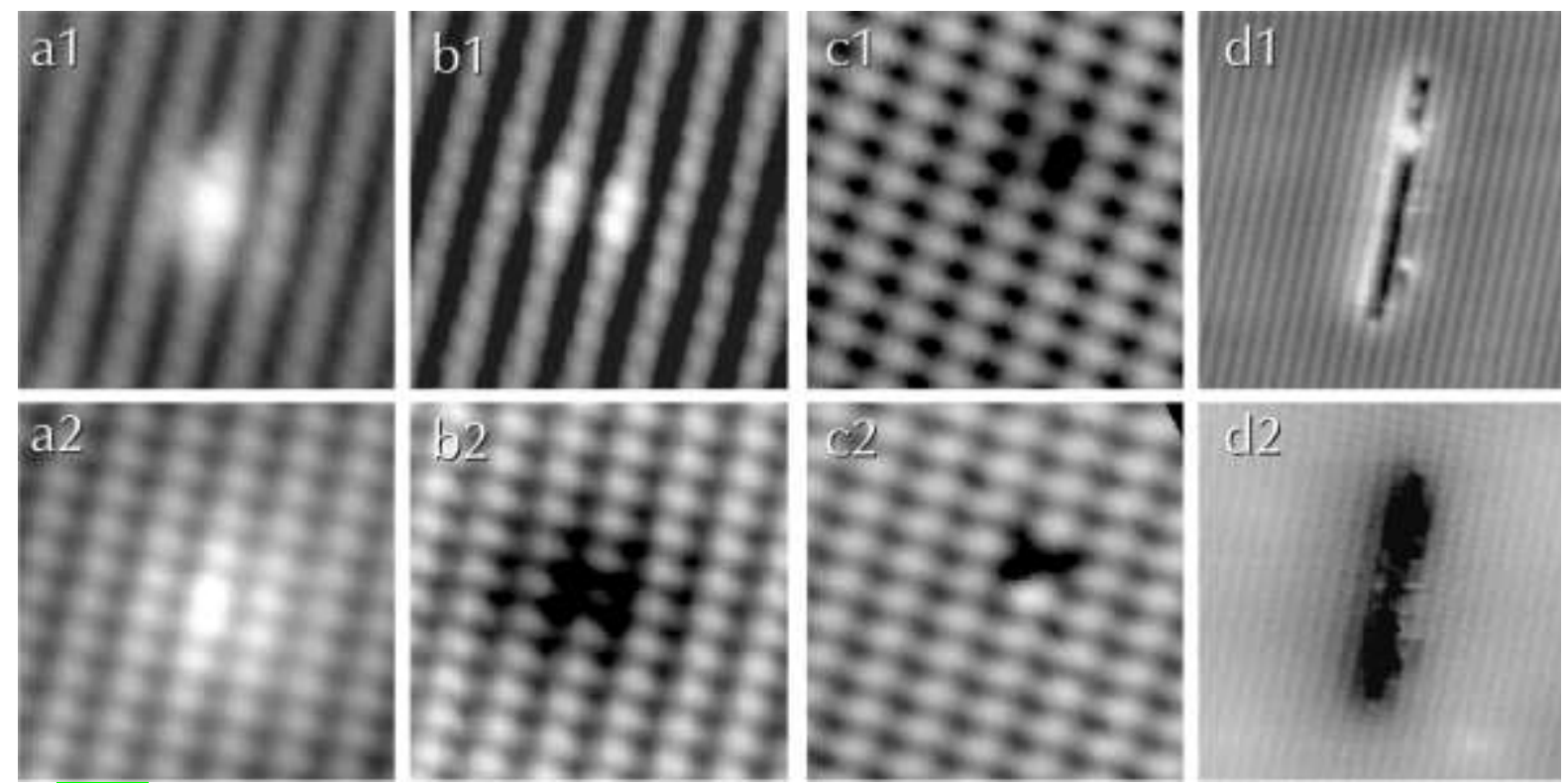

Figure 3: STM images of defects at cleavage surfaces of Si-doped as-grown GaAs bulk crystals. Top row: occupied states images, recorded at biases from -2.0 to -2.2 V. Bottom row: unoccupied states images, recorded at biases from +1.4 to +1.5 V. (a1) and (a2) show a SiGa donor, (b1) and (b2) a SiAs acceptor, (c1) and (c2) a SiGa-Ga-vacancy complex, and (d1) and (d2) the intersection line of a planar Si cluster. (Reprinted figure with permission from Reference [10] Copyright (1996) by the American Physical Society).

and illustrate why - for example - a sub-surface impurity atom which may have little or no impact on the surface height can nonetheless impact the STM data via its impact on the local electronic structure. Within our understanding of STM images as mapping the LDOS, we can also understand the opportunities offered by imaging at different tip biases. For a tip positively biased relative to the sample, electrons flow from the occupied states of the sample into the unoccupied states of the tip. Reversing the polarity of the bias instead accesses the unoccupied states of the sample. When imaging compound semiconductors, the electronegativity difference between the metallic and non-metallic species can 
allow images of the occupied states of the sample to be dominated by the electron-rich non-metallic sites, whereas images of the unoccupied states primarily address the metallic sites. This ability to distinguish between the metallic and non-metallic sublattices can allow identification of, for instance, which sublattice accommodates a particular amphoteric dopant atom: for example in highly Si-doped $\mathrm{GaAs}, \mathrm{Si}_{\mathrm{Ga}}$ donors can be observed, but so can $\mathrm{Si}_{\mathrm{As}}$ acceptors, $\mathrm{Si}$ donor-Ga vacancy complexes ( $\mathrm{Si}_{\mathrm{Ga}^{-}}$ $\mathrm{V}_{\mathrm{Ga}}$ ) and planar Si clusters on (111) planes [10], all of which act as compensating defects reducing the overall electron concentration which can be achieved (Figure 3).

Additional insights into the impact of defects on electronic structure can be gained using scanning tunneling spectroscopy (STS): the recording of site-specific current-voltage $(I-V)$ or $\mathrm{d} I / \mathrm{d} V-V$ curves. Under conditions where electrons are injected from the occupied states of the tip into the unoccupied states of the sample, peaks in the $\mathrm{d} I / \mathrm{d} V-V$ curve, can (at a basic level) interpreted as corresponding to energies with a high LDOS. More detailed interpretation of such curves can be challenging, and this approach is less widely applied than voltage dependent imaging, but as early as 1993 this technique was successfully used to reveal donor levels associated with bulk antisite defects in GaAs. Later studies address a wide range of samples [8], including 2D materials such as graphene [11].

\subsubsection{Scanning electron microscopy}

\subsubsection{Brief introduction to SEM}

In a scanning electron microscope (SEM) an image is formed when the intensity of the resultant electrons, X-rays, light or current is monitored as a focussed electron beam is rastered over the surface of the sample. In the scanning electron microscope the energy of the electron beam is usually selected from a range of energies spanning $100 \mathrm{eV}$ to $30 \mathrm{keV}[12,13,14,15]$. Figure 4 shows a schematic of an SEM and also illustrates the range of signals which can be used to generate images and highlights those signals which can reveal defects present in the sample. 


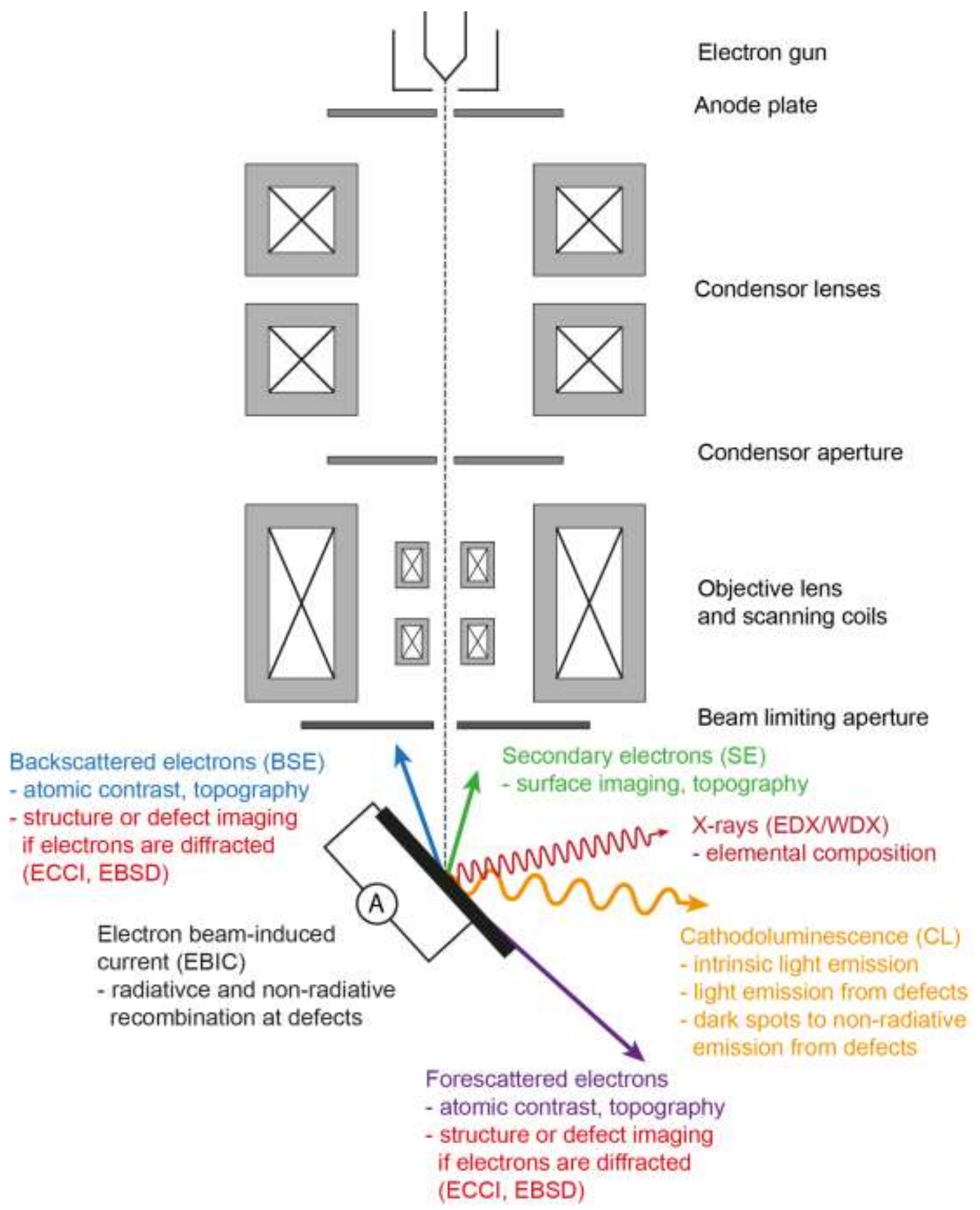

Figure 4: Schematic of an SEM and an illustration of the range of signals which can be obtained.

\section{Resolution}

The spatial resolution of the images generated in the SEM depends on the electron beam spot size which is dependent on the size of the electron beam source; the electron energy; the beam current; and electron optical aberrations, in particular chromatic (due to the range of energies emitted by the electron source) and spherical aberrations and astigmatism. It also depends on the interaction volume; that is the volume within the sample from which the signal is obtained. The interaction volume depends on the signal being measured as displayed in Figure 5; the atomic number and density of the material under investigation; the energy of the incident beam; and the angle of the incident beam. For light and current based measurements, resolution may also be determined by carrier diffusion [16,17].

The size of the electron source and the energy spread depends on the type of electron gun is used. Table 1 summarises the types of guns available and their typical properties [13,14,15,18]. For imaging defects a small source size, low energy spread, low beam divergence and high beam current/beam brightness is 
required. In addition, for the imaging of defects using diffraction imaging, usually referred to as electron channelling contrast imaging (ECCI), a beam with low divergence is required. This will be discussed in section 10.2.2.2. An SEM with a Schottky emitter is the instrument best suited for imaging and analysing defects. The resolution achievable for different imaging modes will be discussed later in this section.

\begin{tabular}{|c|c|c|c|c|}
\hline $\begin{array}{l}\text { Electron Gun } \\
\text { Type }\end{array}$ & $\begin{array}{l}\text { Source Size } \\
(\mu \mathrm{m})\end{array}$ & $\begin{array}{l}\text { Energy Spread } \\
(\mathrm{eV})\end{array}$ & $\begin{array}{l}\text { Beam current } \\
\text { (nA) } \\
(\text { at } 20 \mathrm{keV})\end{array}$ & $\begin{array}{l}\text { Beam } \\
\text { brightness } \\
\left(\mathrm{A} / \mathrm{cm}^{2} / \mathrm{str}\right) \\
(\text { at } 20 \mathrm{keV})\end{array}$ \\
\hline $\begin{array}{l}\text { Tungsten hairpin } \\
\text { thermionic emitter }\end{array}$ & $\approx 20$ & $\approx 3$ & $\approx 1000$ & $10^{5}$ \\
\hline $\begin{array}{l}\mathrm{LaB}_{6} \text { thermionic } \\
\text { emitter }\end{array}$ & $\approx 10$ & $\approx 2$ & $\approx 1000$ & $10^{6}$ \\
\hline $\begin{array}{l}\text { Cold cathode field } \\
\text { emitter }\end{array}$ & $\approx 5 \times 10^{-3}$ & $\approx 0.3$ & $\approx 20$ & $10^{8}$ \\
\hline Schottky emitter & $\approx 20 \times 10^{-3}$ & $\approx 0.8$ & $100-500$ & $10^{8}$ \\
\hline
\end{tabular}

Table 1. Summary of electron guns used in scanning electron microscopes $[13,14,15,18]$

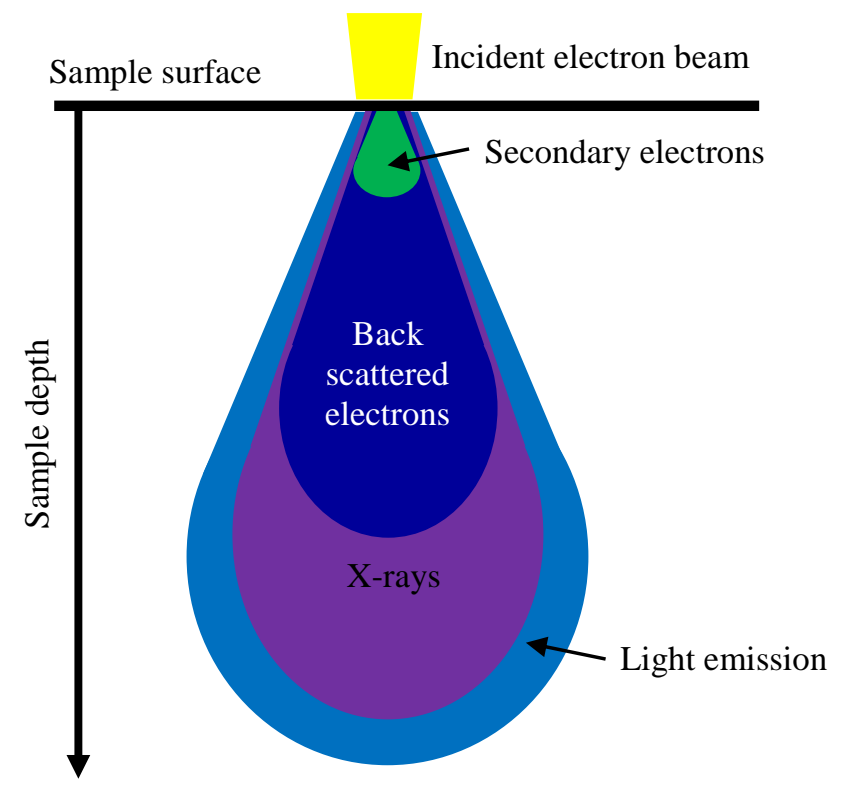

Figure 5: Illustrating the signals detected in the SEM. 


\section{Signals used to generate images in the SEM}

The signals which are used to generate images in the SEM are illustrated in Figure 4 and are described below. Figure 5 illustrates schematically the interaction volume for each of these signals.

\section{Secondary electrons}

The interaction of the electron beam with the sample gives rise to the emission of secondary electrons through ionisation of the sample under study. Secondary electrons are low energy electrons and are defined as those electrons with energy less than $50 \mathrm{eV}$. When the intensity of secondary electrons is monitored as the beam is scanned over the sample, a topographic image of the surface of the sample is formed. A topographic image is produced because the intensity of the secondary electron signal is dependent on the orientation of the sample surface with respect to the incident electron beam and to the position of the secondary electron detector. There is a higher yield of secondary electrons from a surface inclined with respect to the electron beam when compared to that of a flat surface, and regions of the sample facing the detector will appear brighter than those facing away. Because of their low energy, secondary electrons have a high probability of re-absorption in the sample. Therefore, only those generated close to the surface are emitted. Secondary electron imaging is therefore an extremely surface sensitive imaging mode (i.e., the interaction volume is small) and images with a resolution of order 1 $\mathrm{nm}$ can be obtained in an SEM with a cold cathode field emitter or Schottky emitter. Figure 6 shows a secondary electron image revealing the topography of a nanoscale GaN pyramid structure. Note the edges of the pyramids are bright which is due to more secondary electron emission from an edge when compared to a flat surface.

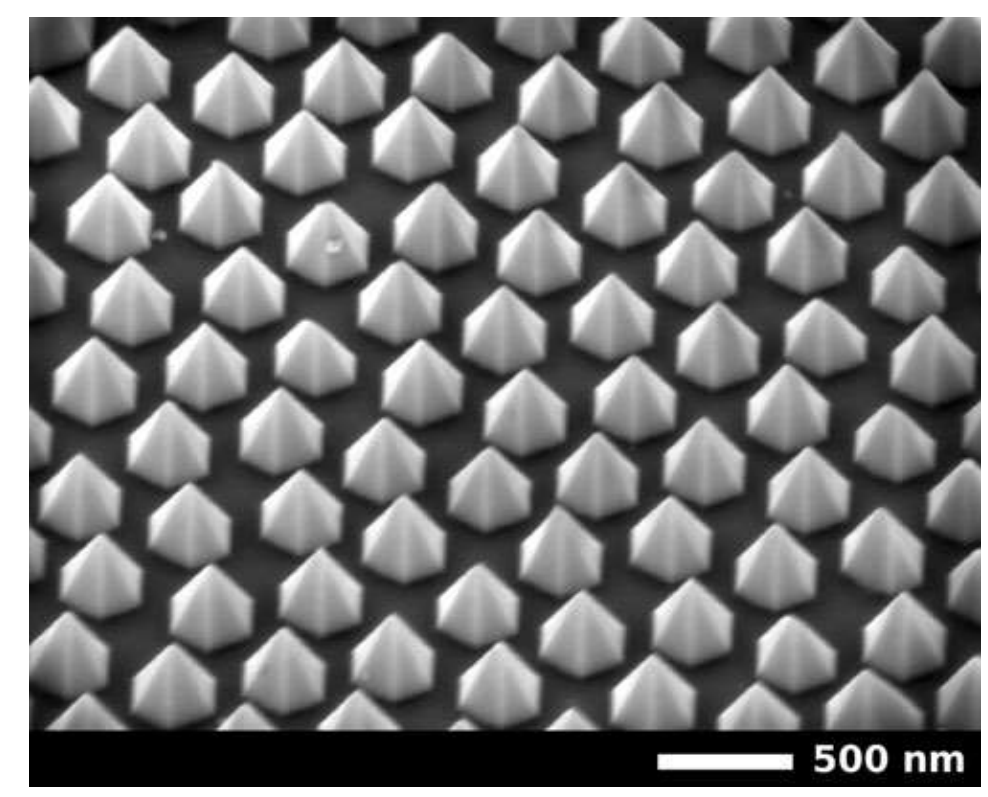

Figure 6: Secondary electron image of an array of GaN-based nanopyramids (reprinted from [19] with permission from Cambridge University Press).

Secondary electrons are typically detected by an Everhart-Thornley detector. This detector comprises an electron collector which is positively biased at a voltage of around $200 \mathrm{~V}$ to attract the secondary electrons; and a scintillator biased at around $10 \mathrm{keV}$ to convert the electrons to light which is coupled to a photomultiplier tube. Secondary electrons may also be detected by an in-lens detector where the secondary electrons are collected through the objective lens of the SEM.

\section{Backscattered electrons}

Backscattered electrons are those which have been elastically or inelastically scattered from the sample. Their energy can range from that of the incident beam down to that of the secondary electrons. Figure 7 illustrates the energy distribution of secondary electrons and backscattered electrons in the SEM. The backscattered yield is dependent on the atomic number and density of the material under study, so atomic contrast can be provided by this imaging mode. The backscattered yield is also dependent on the inclination of the sample surface so backscattered electrons can also be used to provide an image of 
surface topography. As backscattered electrons generally come from deeper within the sample than secondary electrons, backscattered electron images generally exhibit a lower resolution than secondary electron images.

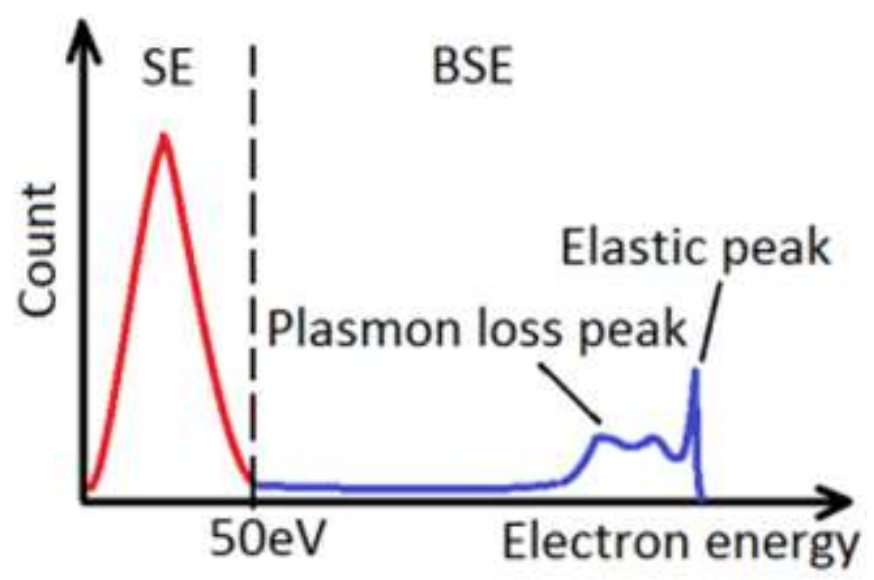

Figure 7: Schematic of the electron energy spectra (reprinted from[20] under Creative Commons Attribution (CC BY) license: http://creativecommons.org/licenses/by/4.0/).).

Backscattered electrons may also be detected by an Everhart-Thornley detector. In this case the electron collector is negatively biased (at around $-50 \mathrm{~V}$ ) to repel the lower energy secondary electrons. Backscattered electrons may also be detected by an electron sensitive diode(s) placed underneath the final SEM lens (pole pieces). This is usually a four quadrant diode, if the signals from all the quadrants are added together, a compositional contrast dominates; if the quadrants are operated in adjacent pairs and the signal from one pair subtracted from the other, topographic contrast dominates.

Where the electrons incident on the sample undergo diffraction and an image formed by monitoring the intensity of the backscattered electrons as the beam is rastered over the sample, an image exhibiting contrast related to the sample's structural properties may be formed. This imaging modality is commonly referred to as electron channelling contrast imaging (ECCI)). Where the electrons exiting the sample undergo diffraction, and the resultant electron backscatter diffraction (EBSD) patterns are recorded at each point in the raster scan, information derived from each EBSD pattern can be used to produce a map revealing the sample's structural properties. This mapping technique commonly referred to as electron backscatter diffraction (EBSD) mapping. These techniques are discussed in the sections below.

\subsubsection{Electron channelling contrast imaging}

Electron channelling contrast images are produced when a sample is positioned so that a plane or planes in the sample are at, or are close to, the Bragg angle. That is the electrons entering the sample are diffracted and it is this which provides the dominant structural contrast in the resulting image. Electrons diffracted on exiting the sample can contribute to the structural contrast, but this component is highly dependent on the position of the detector. Any deviation in crystallographic orientation or in lattice constant due to local strain, may then be revealed by variation in the contrast in the electron channelling image constructed by monitoring the intensity of backscattered or forescattered electrons as the electron beam is scanned over the sample. Extremely small changes in orientation and strain are detectable, revealing, for example, low angle tilt and rotation boundaries and atomic steps and enabling extended defects such as dislocations and stacking faults to be imaged [21,22,23,24,25,26,27,28,29]. ECCI can provide similar information on defects as the transmission electron microscope (see sections 10.2.3 and 10.3.1.4) where the defects either thread to the surface or lie within around $50 \mathrm{~nm}$ of the surface.

For successful imaging of the structural properties of materials using ECCI all that is required is that the sample has a reasonably smooth, clean surface. For the imaging of metal surfaces this usually requires careful sample polishing to produce a high quality surface [30], this is also the case for semiconductor wafers cut from bulk crystals. However for most epitaxially grown semiconductor thin films no surface preparation is required. The spatial and depth resolution of ECCI is of the order of tens of nanometres. Figure 8 illustrates the two geometries, namely the backscatter and forescatter geometries which are used to acquire ECCI micrographs. The backscatter geometry (Figure 8(a)) has the advantage that this geometry does not require a high tilt of the sample and therefore a significant correction of the image to account for tilt is not required. This geometry also allows the easiest imaging of large samples, for 
example full semiconductor wafers. The forescatter geometry (Figure $8(\mathrm{~b})$ ) has the advantage that images exhibit better signal-to-noise with respect to the backscatter geometry due to the increase in intensity of backscattered electrons. The detector used to detect the backscattered electrons is generally an electron-sensitive diode. Key to the acquisition of good quality ECCI micrographs is the use of a good amplification system.
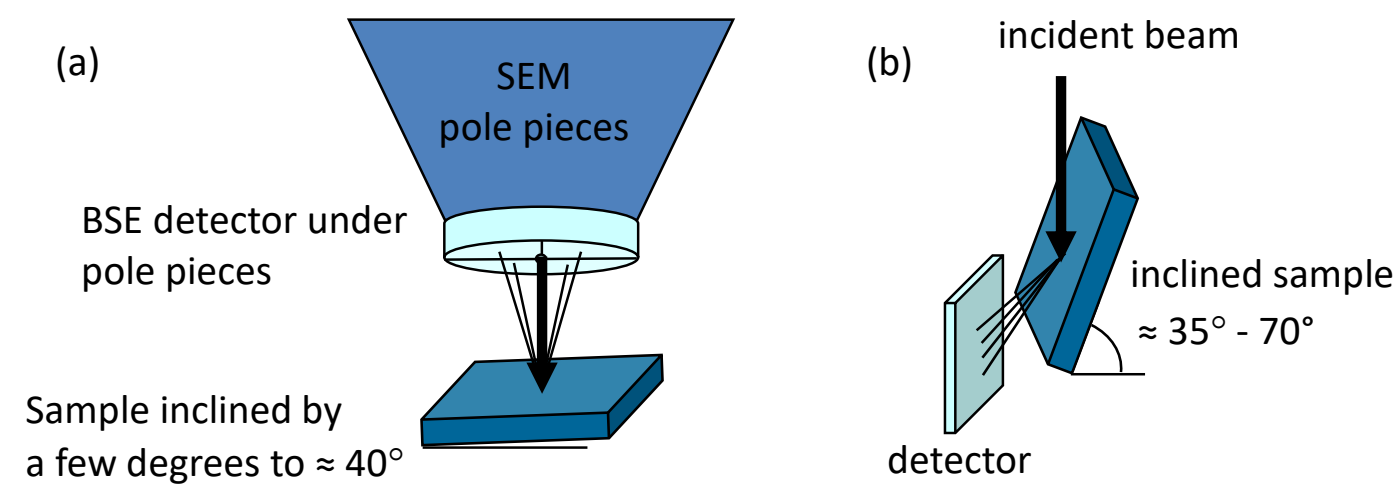

Figure 8: Illustrating the (a) backscatter and forescatter geometries for acquisition of ECCI micrographs.
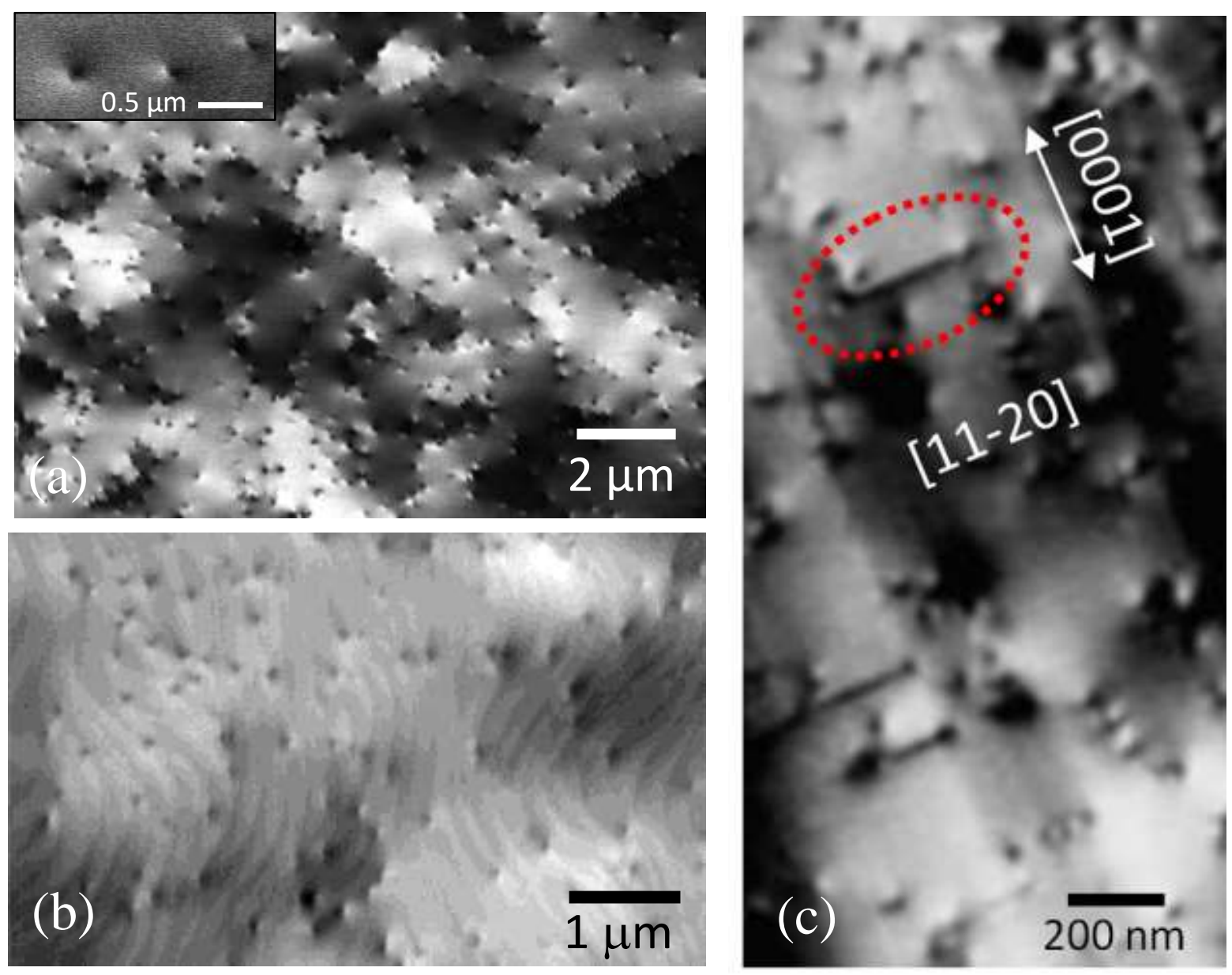

Figure 9: (a) ECCI micrograph of a GaN thin film showing threading dislocations. Inset: an enlarged image of three dislocations. The different grey levels in the image indicate areas of different orientation (reprinted from [65] with permission from Cambridge University Press). (b) ECCI micrograph of a c-plane GaN thin film showing threading dislocations and atomic steps (reprinted from [22] with permission from the American Physical Society). (c) ECCI micrograph of an m-plane GaN thin film showing stacking faults (one highlighted by the ellipse). Partial dislocations can also be seen at the ends of the stacking faults (image courtesy of G. Naresh-Kumar). 
Figure 9 shows electron channelling contrast images from nitride semiconductor thin films. All the images (and the inset of Figure 9(a)) show threading dislocations as spots with the black-white (B-W) contrast related to the strain field associated with the dislocations. Figure 9(b) shows an ECCI micrograph revealing atomic steps. Figure 9(c) shows stacking faults and associated partial dislocations on either end of the stacking fault. A simple geometric procedure has been developed to identify a given threading dislocation in c-plane nitride thin films as either edge, screw, or mixed type by exploiting differences in the direction of the black-white contrast between two ECCI micrographs acquired under quasi 2-beam conditions from two symmetrically equivalent crystal planes. Crystal planes were chosen for which the diffraction vectors $\left(\mathbf{g}_{1}\right.$ and $\left.\mathbf{g}_{2}\right)$ were at $120^{\circ}$ to each other, where the $\mathbf{g}$-vector was determined through the acquisition of electron channelling contrast patterns (ECPs) [22].

An ECP is obtained when changes in the backscattered electron intensity are recorded as the angle of the incident electron beam is changed relative to the surface of a single crystal area of the sample. When a backscattered image is acquired at low magnification, as the beam is scanned over the sample, it changes its angle with respect to the surface of the sample (in our case by around $\pm 2.5^{\circ}$ ), allowing an ECP to be obtained [22]. Note that this method of acquiring an ECP is only possible if the scanned area of the sample (of order $5 \mathrm{~mm} \times 5 \mathrm{~mm}$ in size) is smooth and of the same crystallographic orientation. Alternatively, if 'beam rocking' electron optics are available in the SEM, an ECP can be acquired from an area ranging from of order $10 \mu \mathrm{m} \times 10 \mu \mathrm{m}$ to of order $500 \mathrm{~nm} \times 500 \mathrm{~nm}$ in size [31], such ECPs are often referred to as selected area ECPs (SAECPs or SACPs). When the beam changes its angle with respect to the sample, different planes of the crystal satisfy the Bragg condition, giving rise to the appearance of overlapping bands of bright and dark lines (Kikuchi lines) superimposed on the image of the sample; an ECP from a GaN thin film is shown in Figure 10. The ECP is a 2D projection of the crystal structure, with the Kikuchi lines corresponding to different planes in the crystal. Comparing the ECP with kinematical and/or dynamical electron diffraction simulations allows the pattern to be indexed: i.e the planes in the ECP can be identified. The plane (or planes) which intersect the centre of the ECP, usually referred to as the pattern centre (PC), are those from which the incident electron beam is diffracted. In the example shown in Figure 10, the incident electron beam was diffracted from one of the $\{11-20\}$ planes, that is a $\mathbf{g}$-vector of $\langle 11-20\rangle$ was selected.

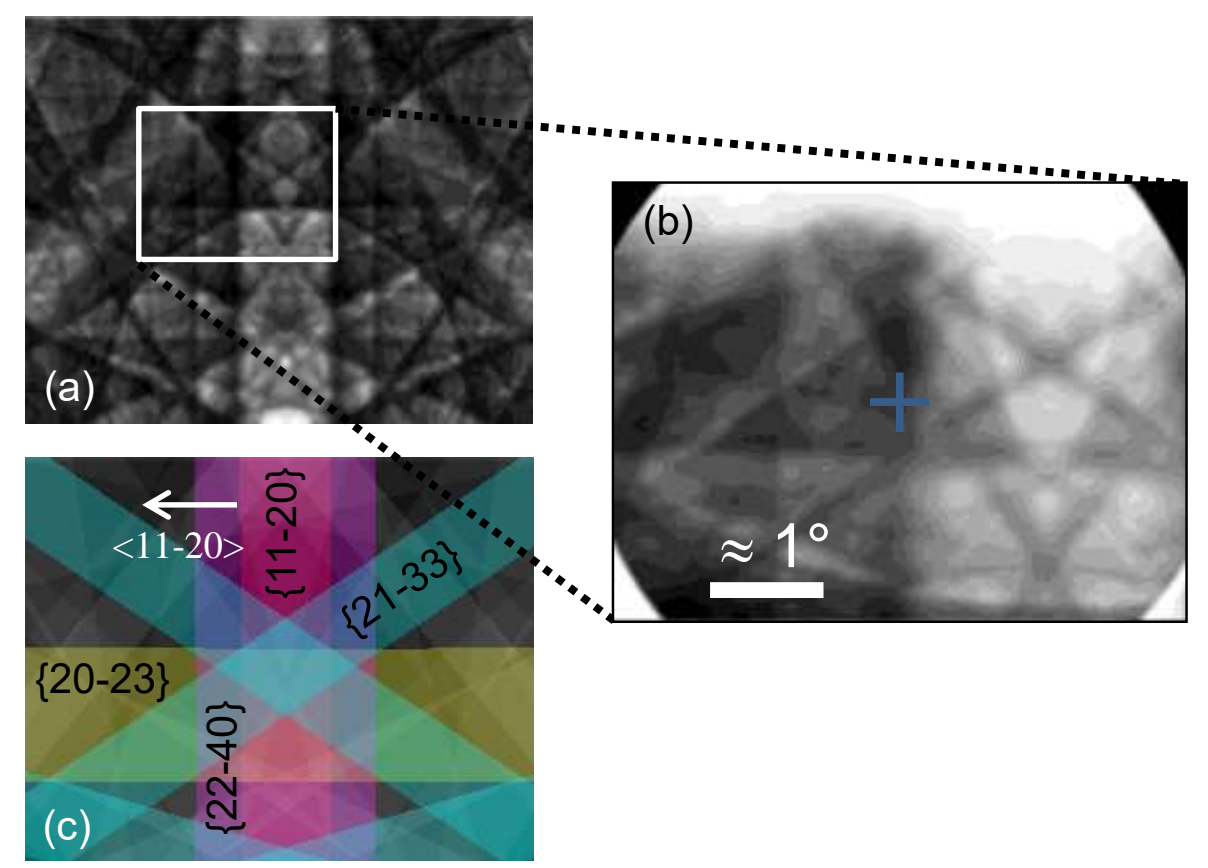

Figure 10: (a) Dynamical simulation of an electron channelling pattern (ECP) from a GaN thin film. Electron beam energy is $30 \mathrm{keV}$, sample tilt $\approx 40^{\circ}$ (courtesy of Aimo Winkelmann). (b) Experimental ECP. The blue cross marks the pattern centre $(P C)(c)$ Kinematical simulation of the ECP with some indexed planes highlighted (produced with ESPRIT DynamicS (Bruker Nano) software). In this case the PC intersects one of the $\{11-20\}$ planes so a $g$-vector of $\langle 11-20\rangle$ was selected.

In principle if the diffraction condition can be selected, defects can be identified by investigating their visibility as a function of the diffraction condition, similar to the technique used to identify defects from 
diffraction images in the TEM (see Section 10.3.1.4). However as ECCI micrographs from semiconductors are usually acquired in a plan view geometry, i.e., from the top of the as-grown surface of the sample, and the collected signal is backscattered electrons as opposed to transmitted electrons, the relaxation of the surface strain associated with a defect plays a significant role with regard to its visibility and this must be taken into account when attempting to identify defects with ECCI.

As mentioned above, threading dislocations in c-plane $\mathrm{GaN}$ can be identified by exploiting differences in the direction of the black-white (B-W) contrast between two ECCI micrographs acquired under quasi 2-beam conditions from two symmetrically equivalent crystal planes. As a result of surface relaxation, the $\mathrm{B}-\mathrm{W}$ contrast direction is perpendicular to the $\mathbf{g}$-vector for a pure screw dislocation [31]. For an edge dislocation the direction of the B-W contrast generally depends on its Burgers vector and not on the $\mathrm{g}$-vector. Hence for edge dislocations, we expect the direction of the $\mathrm{B}-\mathrm{W}$ contrast to either reverse or remain the same when the diffraction conditions are changed. The degree of contrast observed for an edge dislocation, that is its visibility, is dependent on the selected diffraction condition. If the g-vector and the Burgers vector of the edge dislocation are orthogonal, the visibility of the edge dislocation will be strongly reduced and this can help with the identification of the dislocation. If the diffraction conditions are selected so that the contrast observed for both edge and screw dislocations are similar, mixed dislocations may be identified as their B-W contrast direction changes in a manner intermediate to that of the edge and screw dislocations. In practice it is difficult to achieve exact 2-beam conditions; however it is possible to get sufficiently close to allow quantitative analysis of the resultant ECCI micrographs. Where it is not possible to acquire an ECP, it is possible to identify the threading dislocations as either an edge dislocation or one with a screw component (i.e., a screw or a mixed dislocation) by comparing two ECCI micrographs acquired for the sample placed in at least two orientations. If, for a given dislocation, the $\mathrm{B}-\mathrm{W}$ contrast direction 'flips' (rotates by $180^{\circ}$ ), or stays the same, on comparison of the two images, then it is likely to be an edge dislocation. The B-W contrast direction for a screw or mixed dislocation will be observed to change but by an angle other than $180^{\circ}$. In practice the direction of the $\mathrm{B}-\mathrm{W}$ for each dislocation should be compared for at least three images to unambiguously identify a dislocation as either an edge dislocation or one containing a screw component.

Simulations of dislocation contrast may also help the understanding of the contrast exhibited by dislocations observed in ECCI micrographs. For example Carnevale et al. [27] used simulations to support their analysis of misfit dislocations in thin films of $\mathrm{GaP}$ grown on $\mathrm{Si}$, while Picard et al. have simulated contrast for inclined threading edge and screw dislocations in $\mathrm{SrTiO}_{3}$ [32] and Pascal et al. have simulated contrast for threading edge dislocations in $\mathrm{GaN}$ [33].

The conditions required to image defects using ECCI are quite stringent: a high brightness, small beam diameter (nanometres), high current (nanoamps or higher), low divergence (a few mrad) electron beam, is required. As discussed above, such conditions are met in an SEM equipped with a Schottky gun. ECCI micrographs are generally acquired at electron beam energies of $20 \mathrm{keV}$ to $30 \mathrm{keV}$ to obtain the best signal-to-noise ratio. The performance of the diodes used to detect backscattered electrons reduces for lower electron beam energies [21]. An amplifier system specifically designed to optimise the contrast and brightness of the ECCI micrographs is also invaluable for obtaining good images. Lastly, ideally the SEM should be equipped with beam rocking to enable the acquisition of SAECPs. The acquisition of electron backscatter diffraction patterns (discussed in the next section) can also help with the determination of diffraction conditions [30].

Until recently, ECCI was mostly used to investigate the structural properties of metals and geological materials, but its use for the characterisation of defects in semiconductors is steadily expanding. To date ECCI has been used to image defects in nitride semiconductors [22,23,24], $\mathrm{Si}_{1-\mathrm{x}} \mathrm{Ge}_{\mathrm{x}}$ [34], $\mathrm{SiC}$ [35], GaAs [26], GaP [27,28], GaAs $\mathrm{P}_{1-\mathrm{y}}$ [28], GaSb [29]. For more information on ECCI, the following papers provide informative reviews of the technique [21,29,34,35].

\subsubsection{Electron backscatter diffraction}

In electron backscatter diffraction (EBSD) the electron beam is incident on a sample which typically is tilted at an angle near to $70^{\circ}$. The primary electrons (typically with energies between $10 \mathrm{keV}$ and 30 $\mathrm{keV}$ ) are scattered inelastically through high angles forming a diverging source of electrons which can be diffracted as they leave the sample. A simple description for the formation of an EBSD pattern (EBSP) presumes that those electrons that satisfy the Bragg condition for a given plane all need to have directions on two so called Kossel cones relative to both the upper and lower surfaces of that plane, see Figure 11(a). The intersections of these Kossel cones with the detector screen approximately mark the 
edges of the Kikuchi bands, which thus have a width of about twice the Bragg angle, with the projection of the relevant lattice plane in the centre of the Kikuchi band. An EBSP consists of a large number of overlapping Kikuchi bands and is related to a 2-D, gnomonic, projection of the crystal structure, see Figure 11(b). The Kikuchi lines appear as almost straight lines, because the Kossel cones are very shallow, as the Bragg angle is of order $1^{\circ}$. Each Kikuchi band is effectively a trace of the lattice plane from which it is formed; an EBSD pattern can thus provide a direct measurement of the angles between lattice planes and directions in a sample's crystal structure.
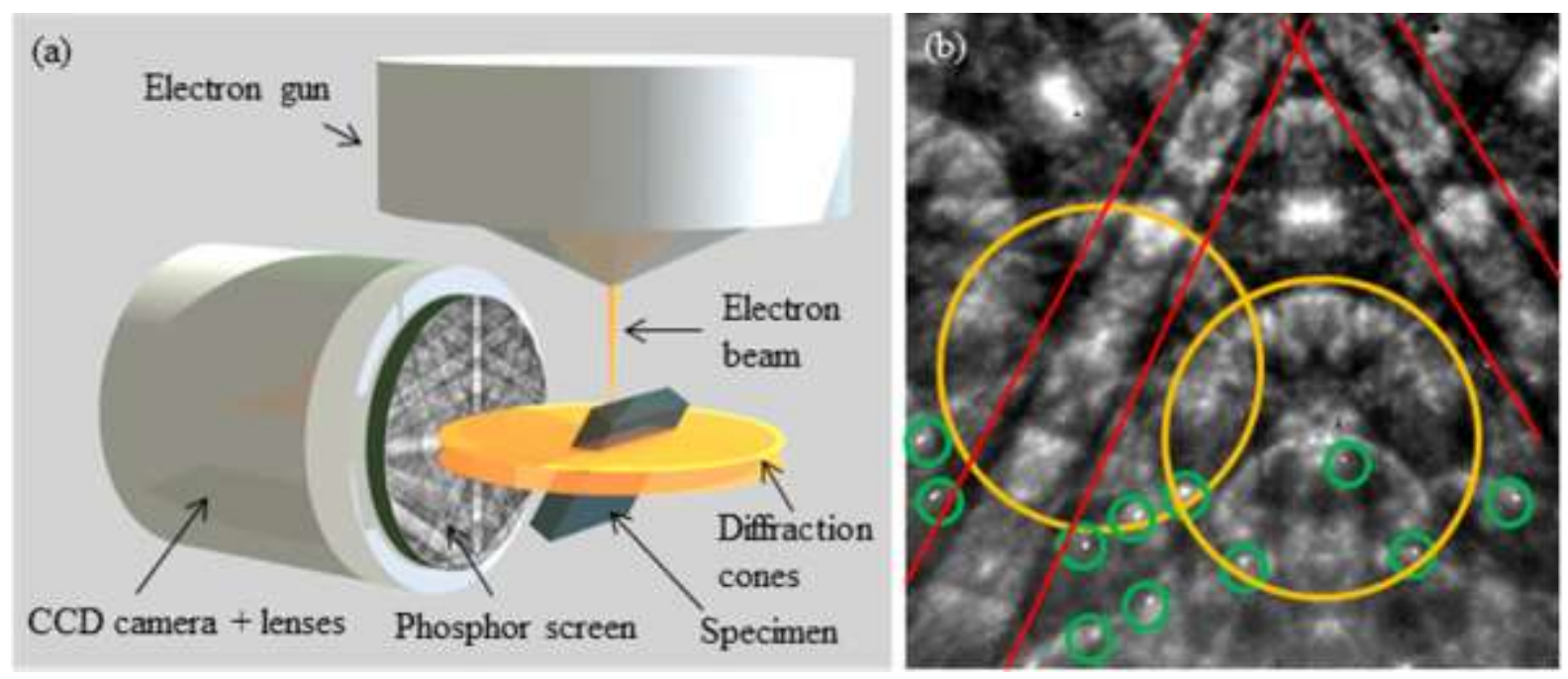

Figure 11: (a) Illustration of the EBSD detection geometry and a conventional EBSD detector. (b) An EBSP from a GaN thin film acquired at an energy of $5 \mathrm{keV}$ and a probe current of $\approx 1.5 \mathrm{nA}$. The red lines outline a pair of Kikuchi bands; the large yellow circles indicate high-order Laue zone (HOLZ) rings and the small green circles highlight reflection highenergy electron (RHEED) spots. Reprinted from [38] under Creative Commons Attribution (CC BY) license: http://creativecommons.org/licenses/by/4.0/).

The EBSP is generally detected by an electron sensitive phosphor or scintillator screen and a CCD or CMOS camera [36] (see Figure 11(a)), although there have been recent developments of direct electron cameras [37,38]. The EBSP shown in Figure 11(b) was acquired at $5 \mathrm{keV}$ from a GaN thin film using energy-filtered direct electron detection. Direct electron detection allows high quality EBSPs to be acquired at low electron beam energies, pattern acquisition down to $3 \mathrm{keV}$ is achievable [38].

Rotations of a crystal (e.g. tilts or twists in a thin film) will produce corresponding rotations of the EBSP; while strain will produce a distortion of the EBSP. Therefore, if EBSPs are acquired from a grid of points on a sample, it is possible to analyse these EBSPs to produce maps of tilts, twists and strains. For a more detailed discussion of the EBSD technique see [21,36,39].

Recent developments of EBSD analysis techniques, where experimental EBSD patterns are compared with either a reference experimental pattern or simulated pattern using cross-correlation, have greatly improved the sensitivity of EBSD and its applicability to the study of semiconductors. Sensitivities of less than one part in 10,000 to changes in tilt, in-plane rotation and strain are now possible [40]. Once such sensitivity is achievable it is then possible to calculate the density of dislocations necessary to accommodate the observed misorientations and strain, that is the geometrically necessary dislocations (GNDs) [41]. For an example of what can be achieved with such cross-correlation high resolution electron backscatter diffraction (cross-correlation HR-EBSD), Figure 12(a) shows an EBSD-derived twist map for a patterned epitaxially overgrown $\mathrm{GaN}$ thin film [42]. Figure 12(b) shows the distribution of GNDs derived from the measurement of tilt, twist and strain. 

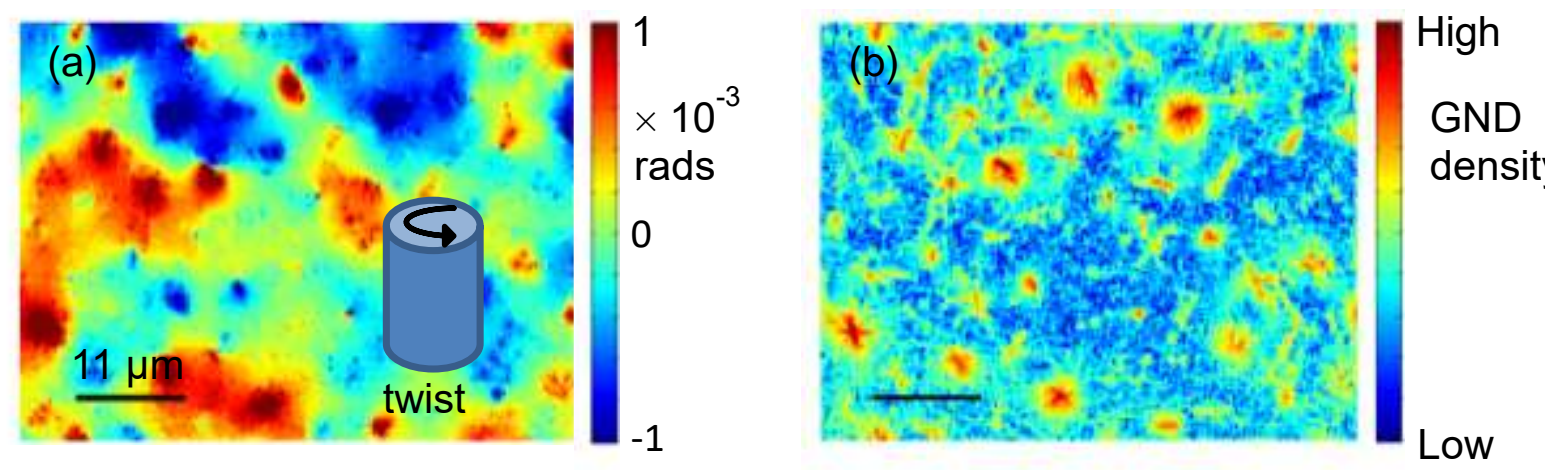

Figure 12: (a) A cross-correlation HR-EBSD map of twist from a patterned epitaxially overgrown GaN thin film. (b) GND distributions derived from the measurement of tilt, twist and strain using cross-correlation HR-EBSD. Maps courtesy of A.Vilalta-Clemente, A. J. Wilkinson and G. Naresh-Kumar.

As for ECCI, EBSD has mostly been used to investigate the structural properties of metals and geological materials, however with the development of cross-correlation HR-EBSD, its use for the characterisation of semiconductors is steadily expanding. For example it has been used to study crystal orientation and/or strain in Si [43], Ge [44], SiGe [45] and nitride semiconductors [42,22,46,47]. A detailed description of this technique for the study of InAIN thin films and its correlation with ECCI can be found in [47].

The cross-correlation of experimental EBSD patterns with simulated patterns has also enabled EBSD to reveal the presence of polytypes e.g., wurtzite and zincblende $\mathrm{GaN}$ in the same sample [48], antiphase domains [49], or inversion twinning [50]. For example Figure 13(a) shows an EBSD-derived map of a $\mathrm{GaP}$ thin film containing antiphase domains [49]. Antiphase domains arise in zincblende-type crystals due to their non-centrosymmetric nature. The zincblende crystal structure is not invariant to a rotation of $90^{\circ}$ around the [001] direction, because the arrangement of the anions and cations in the (111) plane is inverted relative to that of the (1-11) plane; this is illustrated in Figure 13(b). Figure 13(a) shows an EBSD-derived map from a GaP thin film revealing domains (green and blue) which are rotated by 90 degrees relative to each other around the [001] surface normal, corresponding to an interchange between the [111] and [1-11] directions. The orientation of each point of the map was obtained from crosscorrelation of the acquired EBSD pattern with simulated patterns. The alignment of the antiphase domains along the $\langle 110\rangle$ direction is due to a small miscut of the Si substrate [49].
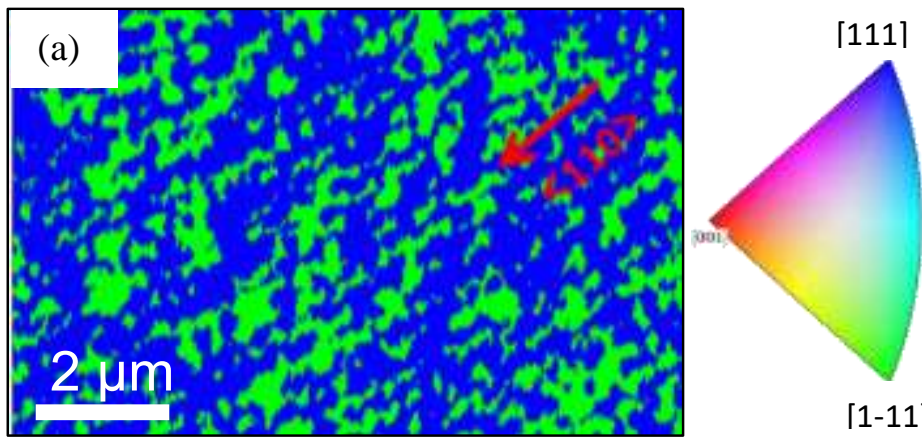

$\lceil 1-11\rceil$ (b)
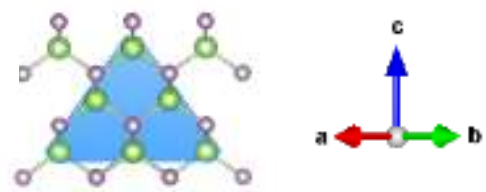

(111)

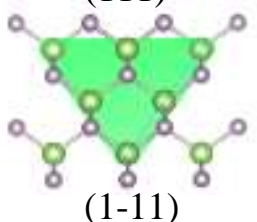

Figure 13: (a) EBSD-derived map revealing regions exhibiting different orientations of the zincblende lattice with respect to the [001] direction in a GaP thin film (antiphase domains). The antiphase domains are identified from crosscorrelation of each experimental pattern in the map with dynamical simulations (reprinted from [49] under Creative Commons Attribution (CC BY) license: http://creativecommons.org/licenses/by/4.0/). (b) Illustrating the different stacking of the Ga and phosphorous atoms in the GaP lattice for the (111) and (1-11). The images were generated using VESTA 3 [51].

\subsubsection{Cathodoluminescence}

Cathodoluminescence (CL) is the process of light generation by an impinging beam of electrons on a material [52]. CL reveals the influence of crystal structure, composition, and strain on intrinsic luminescence and/or reveals defect or dopant-related luminescence. The primary electrons lose energy 
through impact ionisation, which is an inelastic process, generating electron-hole pairs. These pairs can then recombine radiatively generating light emission. It is important to note that in the case of semiconductors the electron energy is a few orders of magnitude larger than the band gap, which makes it possible to even look at wide band gap materials, such as diamond, aluminium nitride (AIN) or boron nitride (BN). There are, however, short wavelength limits imposed on the wavelength detection, such as absorption by water vapour at a wavelengths shorter than $200 \mathrm{~nm}$ (spectrometer and detector are not inside the vacuum system), the material and quantum efficiency of the detector and other optical elements (e.g. absorption of UV light by glass windows or lenses).

The previously mentioned interaction volume (Section 10.2.2.1) defines the lateral dimension and the depth at which the electron beam generates electron-hole pairs. After generation these electron-hole pairs can diffuse before recombination through non-radiative or radiative processes. Their diffusion length increases the volume where the CL signal is generated compared with the volume within which the electron-hole pairs are formed. In $\mathrm{GaN}[53,54]$ the diffusion length of the charge carriers is about 50-60 nm, whereas in GaAs it is can be several microns dependent on the doping concentration [55,56]. The knowledge of the size of the interaction volume and which electron beam parameters influence it are crucial for CL imaging when it comes to depth information and spatial resolution. The interaction volume in a solid can be simulated using Monte Carlo methods, for example CASINO [57] or NIST DTSA-II [58].

The spatial resolution is mostly influenced by the size of the electron beam size, the excitation volume and the carrier diffusion length in the studied material [16]. The highest spatial resolution for bulk material will therefore be ultimately limited by the diffusion length. The diffusion length, though, is a bulk property. Confinement within nanostructures, such as quantum wells, small surface features or defects, further limits the diffusion of the generated carriers by either increasing or decreasing the probability of radiative or non-radiative recombination. This allows features several orders of magnitude smaller than the diffusion length to be observed; for example $20 \mathrm{~nm}$ spaced quantum wells in GaAs have been resolved [59] or peak shifts in the emission from $\mathrm{InGaN} / \mathrm{GaN}$ quantum wells over an $\approx 10 \mathrm{~nm}$ distance have been measured [60]. Other resolution limiting effects include surface contamination by carbon, charging of the sample, mechanical vibrations and electrical noise [59].

Conventional CL imaging either records a panchromatic or monochromatic CL image. For panchromatic $\mathrm{CL}$ the total luminescence intensity is measured, whereas for monochromatic CL the intensity within a specific wavelength interval is chosen. By contrast, CL hyperspectral imaging is a more powerful technique in which an entire luminescence spectrum is recorded at each pixel in an image created by a scanning electron beam $[61,62]$. The resultant multi-dimensional data set can be treated mathematically to extract images describing subsets of the recorded data. For example, numerical peak fitting can produce 2D CL images of the energy peak position, peak area and peak half-width illustrating the spatial luminescence behaviour of the sample under investigation. Another mathematical tool is principal component analysis, a multivariate statistical analysis technique, which is useful for obtaining sample information when dealing with low signal-to-noise spectra [63]. An alternative way to build up a hyperspectral CL data set is by recording a large set of CL intensity images at different wavelengths, where the wavelength is selected by using a monochromator and the light is detected by a photomultiplier tube [64].

Threading dislocations (TDs) which give rise to non-radiative recombination are observed as dark spots in a CL image. If the excitation volume is sufficiently small, the size of the dark spots is determined by the minority carrier diffusion length [65]. Figure 14 shows a CL intensity image from GaN where dislocations are revealed as dark spots. 


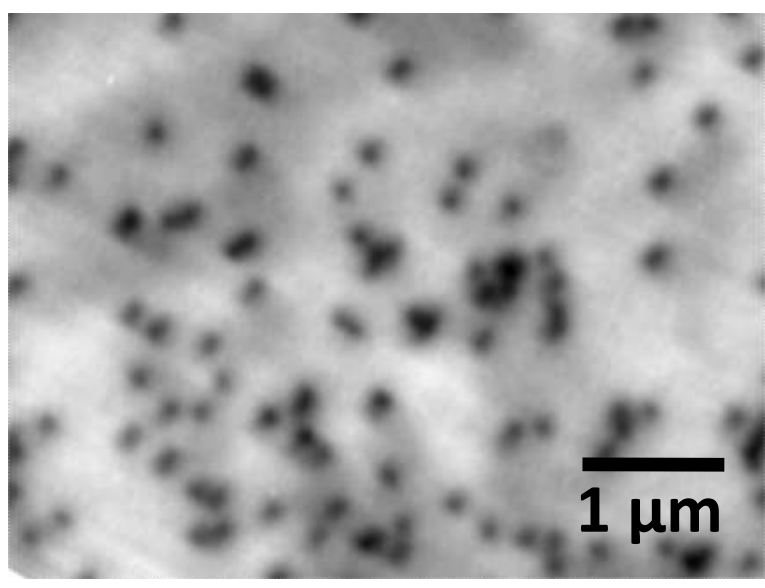

Figure 14: CL intensity image of the near band edge emission in a GaN layer. The dark spots are related to non-radiative recombination at threading dislocations.

Figure 15 shows the CL data obtained through peak fitting from an InGaN/GaN multiple quantum well (MQW) structure. The surface exhibits a high density of V-shaped pits ("V-defects"), common in this type of structure, caused by TDs propagating to the surface and opening up into a pit at one of the QW interfaces. Apart from the previously mentioned non-radiative recombination at the TDs reducing the CL intensity, the peak energy map exhibits a blueshift where a pit is located compared with the surrounding planar areas. This blueshift may be caused by a change in InN content or thinner QW on the facets of the V-pits [60]. The energy shift around the pits is most likely related to strain variations caused by the presence of the dislocations.

Other examples for CL imaging of defects include stacking faults [66,67] and misfit dislocations in GaN [68], dislocations in diamond [69] and GaInP [70] and grain boundaries in silicon [71].

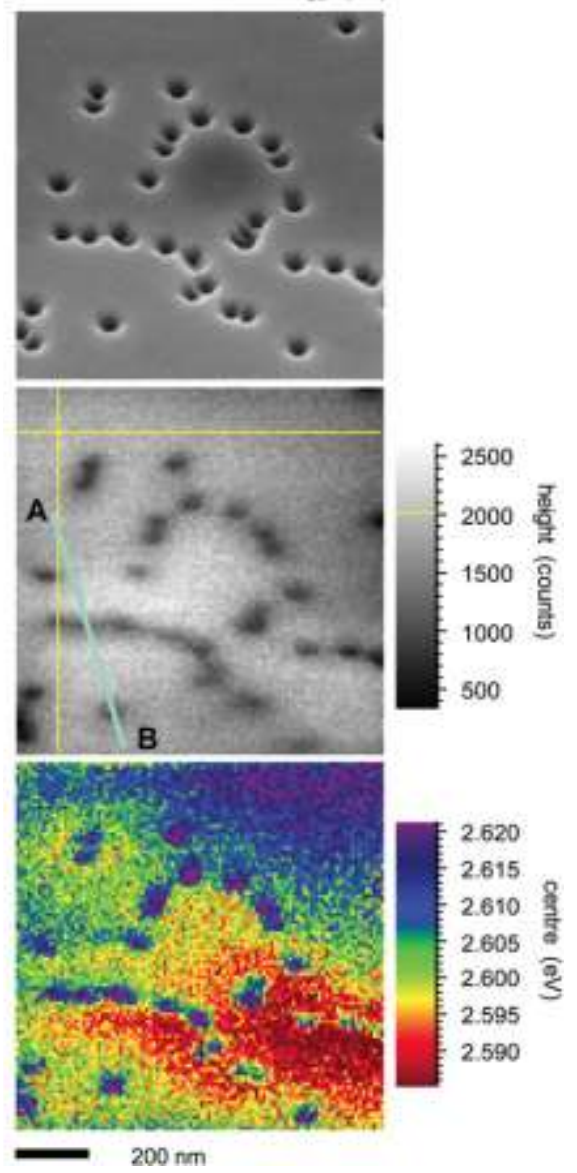

Figure 15: SE micrograph (top), CL intensity (middle) and peak energy image (bottom) of the MQW emission of an In GaN/GaN MQW structure. Adapted and reprinted from [19] with permission from Cambridge University Press. 


\subsubsection{Electron beam induced current}

Electron beam-induced current (EBIC) describes a technique where a measureable current is generated when a high energy electron beam is incident on a sample and electron hole-pairs are created. These electron-hole pairs can undergo three mutually exclusive processes. They can recombine either radiatively (producing cathodoluminescence) or non-radiatively, or they can be swept apart towards electrical contacts and produce a current, which is EBIC [12]. The last process can only occur if there is an internal electric field present in the sample, such as in p-n or Schottky diodes or light-emitting diodes (LEDs), leading to carrier drift towards the contacts. This current can be measured using a current amplifier. Although EBIC is used as a standalone technique for the investigation of recombination properties of defects, it can be combined with CL to provide complementary information [71,72]. The generation of electron-hole pairs can be described by the generation rate $G$ which is a combination of the non-radiative and radiative recombination rates $R_{N R}$ and $R_{R}$, respectively, and the measured current $I_{E B I C}$ :

$$
G=R_{N R}+R_{R}+I_{E B I C} / q
$$

This indirectly provides information on non-radiative recombination processes since the CL provides a measure of the radiative recombination and the EBIC directly measures the current [73]. The resolution of EBIC is similar to CL as discussed in Section 10.2.2.4 since the same electron-hole pairs are involved in this process. However, knowledge of the structure and in particular the depth of the space charge region (caused by the internal electric field) is important for the choice of beam conditions which in turn has an influence on the resolution [17].

An example of EBIC recorded together with the CL of a blue-emitting LED consisting of InGaN/GaN multiple quantum wells (MQWs) is shown in Figure 16 [74]. The CL intensity image and EBIC image both exhibit darks spots which coincide at the same position. Since the dark spots in the CL image appear in the same location of the corresponding EBIC image, they are not caused by carrier escape from the junction but rather non-radiative recombination at defects, possibly clusters of point defects in the MQW region.
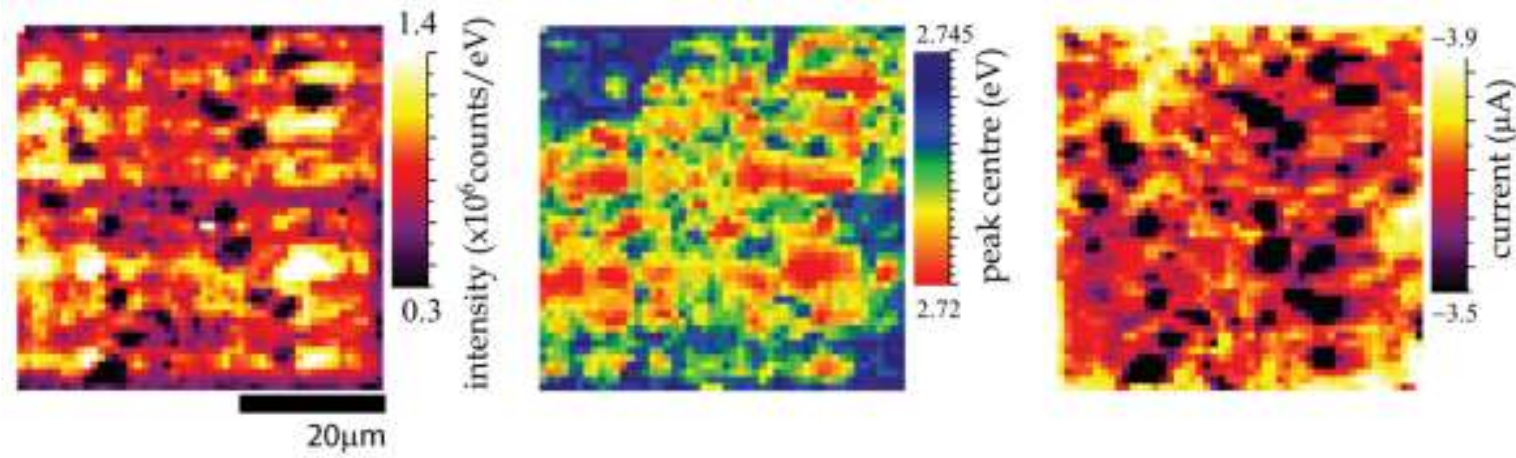

Figure 16: (a) CL intensity and (b) MQW peak energy images and (c) EBIC image of a InGaN/GaN LED. Adapted and reprinted from [74] under Creative Commons Attribution (CC BY) license: http://creativecommons.org/licenses/by/4.0/.

Other examples for EBIC include the investigation of grain boundaries in silicon [71], stacking faults in $4 \mathrm{H}-\mathrm{SiC}$ [75], measurement of the diffusion length in $\mathrm{GaN}$ [76,77] and $\beta-\mathrm{Ga}_{2} \mathrm{O}_{3}$ [78], recombination behaviour of dislocations in bulk $\mathrm{GaN}$ [79] and solar cells [80].

\subsubsection{EDX/WDX}

Energy- and wavelength-dispersive X-ray spectroscopy (EDX/WDX) are two similar techniques for measuring X-rays, which only differ in how the same emitted X-rays are detected. When a high energy electron beam strikes a material it can cause the ejection of an inner shell electron. This hole can then be filled through relaxation of an electron from a higher energetic state. The energy the electron has to lose can result in the emission of a characteristic X-ray. The X-ray energies are specific to the atomic structure of an element and its energy levels [81]. The detection of these X-rays therefore allows elemental identification which is used for compositional analysis of materials [13].

In WDX the X-rays emitted from the material are dispersed to different angles depending on their energy using a diffracting crystal according to Bragg's law. The detector, generally a gas proportional counter 
or scintillation counter, only detects X-rays of one energy at a time and the angle of diffraction has to be changed in order to record a whole X-ray spectrum, similar to a monochromator for light detection. In EDX the X-rays are directly measured using a solid-state silicon detector. The X-rays impinging on the silicon generate electron-holes pairs, the number of which is proportional to the X-ray energy. WDX has a much higher energy resolution compared with EDX, but it is more time consuming to generate an entire X-ray spectrum. EDX on the other hand provides an almost instantaneous X-ray spectrum, but with lower resolution and more noise due to the electronics. A comprehensive review of X-ray spectroscopy and its principles can be found in Goldstein et al.[13 ].

Quantitative measurements of the chemical composition of identified elements are generally carried out by WDX in an electron probe microanalyser (EPMA), which is similar to an SEM, but a dedicated machine with numerous WDX spectrometers containing different diffracting crystal in order to cover a range of X-ray energies. For quantitative analysis the results of the sample under investigation have to be compared against standards of known composition and matrix corrections need to be applied taking additional effects (e.g. material density, absorption, energy loss) into account that could lead to errors in the results [12]. The spatial resolution mainly depends on the size of the excitation volume, which is defined by the electron beam energy. This means that there is a trade-off between the highest spatial resolution and the necessary, minimum beam energy for excitation of X-rays [82].

Figure 17(a) shows an example of a composition image of the In:Ga ratio, which was calculated from WDX elemental images of indium and gallium for an InGaN epilayer [83]. The CL was simultaneously recorded and CL images of the intensity and peak wavelength of the InGaN near band edge emission are displayed in Figure 17(b) and (c), respectively. Local changes in composition are directly evidenced by an energy shift and intensity change since the band gap is a function of alloy composition as observed in points A and B corresponding to indium-rich and poor regions, respectively.

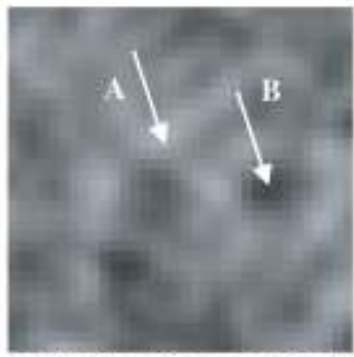

(a) In:Ga ratio, expanded from Fig, I(c)

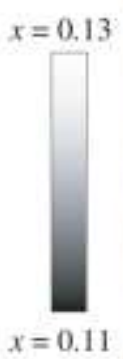

(b) CL counts, expanded from Fig. I(e)

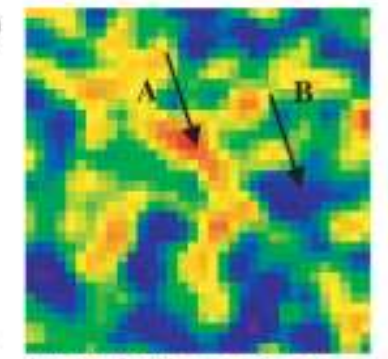

(c) Peak CL wavelength, expanded from Fig. I(f)
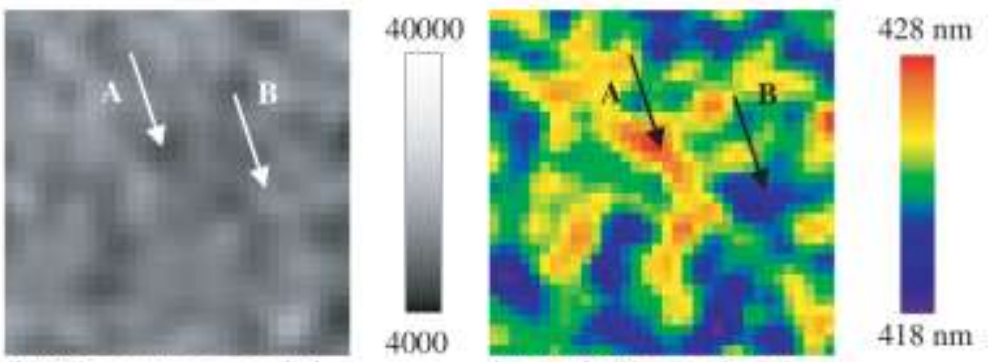

All to same scale: $5 \mu \mathrm{m}$

d Ga WDX maps, (b) CL intensity and (c) peak wavelength maps acquired simultaneously from an InGaN epilayers. Reprinted from [83] with permission form John Wiley and Sons.

\subsubsection{Transmission electron microscopy}

\subsubsection{Brief introduction to TEM}

Electron-matter interaction in a TEM

A transmission electron microscope (TEM), akin to an SEM, is based on the interaction of a beam of electrons accelerated to a high voltage (typically $80-400 \mathrm{kV}$ for a TEM) within a sample. Using a relativistic approach, the wavelength of electrons accelerated to a high voltage is given by the formula

$$
\lambda=\frac{h}{\sqrt{2 m_{0} e V\left(1+\frac{e V}{2 m_{0} c^{2}}\right)}}
$$

where $h=6.626 \times 10^{-34} \mathrm{Js}$ is Planck's constant, $m_{0}=9.109 \times 10^{-31} \mathrm{~kg}$ is the rest mass of the electron, $e=1.602 \times 10^{-19} \mathrm{C}$ is the elementary charge, $\mathrm{V}$ is the accelerating voltage and $c=2.998 \times$ $10^{8} \mathrm{~m} / \mathrm{s}$ is the speed of light in a vacuum. This expression shows that the wavelength, and consequently the theoretical resolution of the microscope, decreases with increasing voltage. For a conventional TEM, with an accelerating voltage of $80-400 \mathrm{kV}$, the wavelength of the electrons ranges between $1.6 \mathrm{pm}$ and $4.2 \mathrm{pm}$ which seems well-suited for the observation of structures at the atomic scale. Nevertheless, it should be noted that in practice it is not the electron wavelength which limits the resolution of the TEM, but instead aberrations in the electromagnetic lenses. In fact, the best microscopes currently used for atomic resolution images employ a rather low voltage (i.e. $60-200 \mathrm{kV}$ ) but some (or several) lens aberrations are corrected. 
An electron, accelerated to a high voltage, will interact with the atoms constituting the sample in several ways, either by interaction with the atomic nucleus or with the electron cloud. The interaction of the incident electron with the nucleus is usually considered to be elastic (i.e. resulting in no loss of energy for the incident electron), whilst interaction with the electron cloud is usually inelastic (i.e. the incident electron transmits part of its energy to the material). Each of these interactions give rise to different signals, which contain information about the material, and which are outlined in Figure 18 and Figure 19. One needs to bear in mind that in TEM, the material exposed to the incident beam consists of a thin foil, as opposed to the bulk material typically imaged in an SEM, and the electrons used in imaging are transmitted through that thin foil. Hence the signals relevant to TEM differ from those outlined for SEM as described in Figure 4 in section 10.2.2.1.

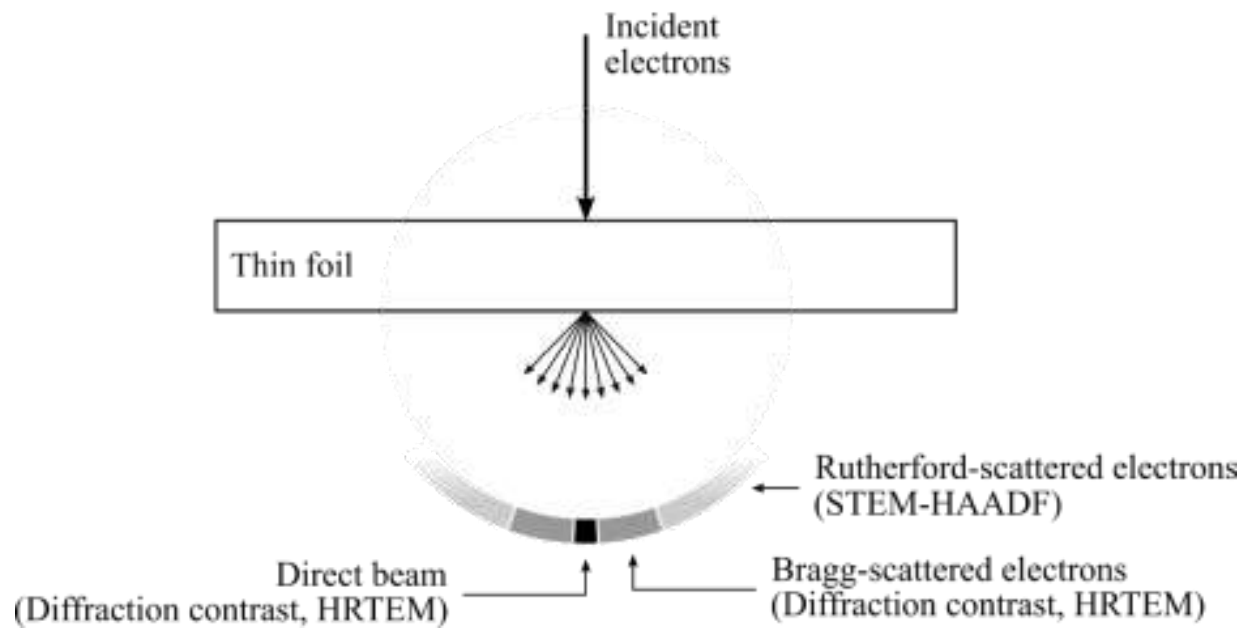

Figure 18: Diagram of the signals generated (and imaging techniques using these signals) by the elastic interaction between a high energy electron beam and a thin sample.

Elastic interaction of an incident electron with the material arises from the interaction of the electrons with the nucleus of the atoms. We will see later that this interaction is not strictly elastic. The incident electron trajectory is then deflected when approaching the nucleus. However, different scattering mechanisms result in an incident electron being deflected to different scattering angles. Hence, the scattering cross-section, $\sigma$, which is the probability of a scattering event occurring, is related to the scattering angle. Because the TEM foil is thin, some of the incident electrons are not scattered and a direct, unscattered beam is transmitted through the sample. Scattering at low angles $(15-50 \mathrm{mrad})$ is dominated by Bragg scattering - which is the same phenomenon used in X-ray diffraction and ECCI as described in section 10.2.2.2. Elastic scattering at such low angles (15-50 mrad) is usually coherent (i.e. the electrons are in phase). Bragg scattering is controlled by the structure of the sample, and results in a diffraction pattern. These electrons will be used for diffraction contrast and high-resolution TEM imaging, as we will describe later. Scattering at higher angles (> $50 \mathrm{mrad}$ ) is usually incoherent and follows the Rutherford scattering rule $\sigma_{R u t h}=1.62 \times 10^{-24} \frac{Z^{2}}{E_{0}^{2}} \operatorname{cotan}^{2}\left(\frac{\theta}{2}\right)$, where $Z$ is the atomic number of the scattering atoms, $\mathrm{E}_{0}$ the incident electron energy, and $\theta$ the deviation angle. Since $\sigma_{R u t h}$ is proportional to $Z^{2}$, Rutherford scattering is exploited in scanning transmission electron microscopy with a high angle annular dark field detector (STEM-HAADF), which provides Z-contrast images as described in section 10.2.3.3. 


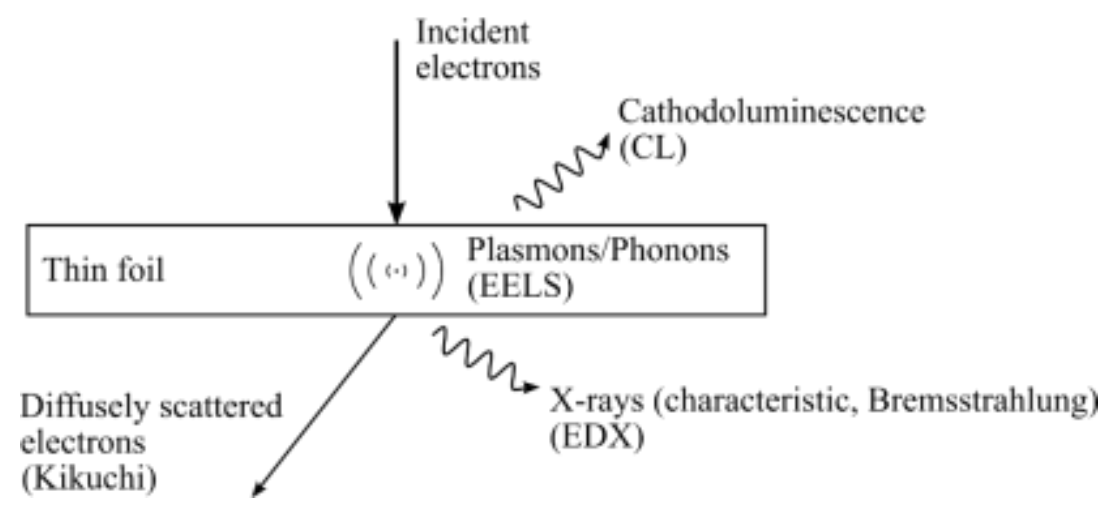

Figure 19: Schematic of the signals generated by the inelastic interaction between a high energy electron beam and a thin sample.

Inelastic scattering corresponds to the interaction of the incident electron with the electron clouds of the sample atoms. The incident electron trajectory is also deviated, but at much lower angles $(<15 \mathrm{mrad})$ than the deviations caused by the nucleus. The energy transferred to the materials can result in various effects, which are summarised in Figure 19. Of relevance in this chapter are the diffusely scattered electrons and X-rays. Diffuse scattering results in the formation of Kikuchi bands, which are very useful when orienting crystalline samples in the TEM. X-rays are generated by electronic transitions in the atoms and are used for energy dispersive X-ray spectroscopy (EDX). Other signals that could be of interest, but not discussed here, are cathodoluminescence (CL) and plasmons and phonons which result in energy losses used in electron energy loss spectroscopy (EELS).

\section{Beam damage}

The high energy electrons used in a TEM can damage the sample. Depending on the type of interaction of the electron with the specimen (elastic or inelastic), different damage mechanisms can occur. Atomic displacement, beam sputtering, specimen heating, specimen charging, structural damage, mass loss and hydrocarbon contamination have been reported in different types of materials [84]. In this section we will pay special attention to the mechanisms of atomic displacement and beam sputtering as they are less straightforward, and therefore more difficult to identify.

Atomic displacement originates from the elastic interaction between an incident electron and the nucleus of an atom in the sample. This interaction, in spite of being considered elastic (as we mentioned earlier), results in a transfer of energy from the electron to the nucleus. This transferred energy, small compared to the electron energy, is given by the formula

$$
E=E_{\max } \sin ^{2}\left(\frac{\theta}{2}\right)
$$

Where $\mathrm{E}$ is the energy transferred to the nucleus (in $\mathrm{eV}$ ), $\mathrm{E}_{\max }$ is the maximum energy that can be transferred corresponding to a $180^{\circ}$ electron deviation (in eV), and $\theta$ is the incident electron trajectory deviation. The maximum energy $\mathrm{E}_{\max }$ can be written as a function of the incident electron energy $\mathrm{E}_{0}$ as follows $[84,85]$.

$$
E_{\max }=E_{0} \frac{E_{0}+2 m_{0} c^{2}}{E_{0}+\left(1+\frac{m_{0}}{M}\right)^{2} \frac{M c^{2}}{2}} \approx 2 E_{0} \frac{E_{0}+2 m_{0} c^{2}}{M c^{2}} \approx E_{0} \frac{1.02+E_{0} 10^{-6}}{465.7 \mathrm{~A}}
$$

where $\mathrm{m}_{0}, \mathrm{M}$ and $\mathrm{A}$ are the rest mass of the electron, the nucleus mass and the atomic mass number of the nucleus, respectively. $\mathrm{E}_{\max }$ is generally rather low - of the order of a few eV - but can reach several tens of eV for light nuclei (e.g. B, C, N, O) and/or high beam energy. Figure 20 illustrates the variation of $\mathrm{E}_{\max }$ with $\mathrm{E}_{0}$ for atoms that compose common semiconductor materials. If the transferred energy exceeds the displacement energy of the nucleus, $E_{d}$, the atom can be moved from its initial location in the crystal, thus creating a Frenkel pair (i.e. a vacancy and an interstitial) in the crystal. The displacement energy $E_{d}$ depends strongly on the nucleus itself, on the nature and strength of the bond of this nucleus in the crystal, and on the crystallographic direction in which the nucleus is to be extracted. The values of displacement $E_{d}$ can often be obtained using molecular dynamics simulations - a rough order of magnitude would be in the range $10-100 \mathrm{eV}$. 


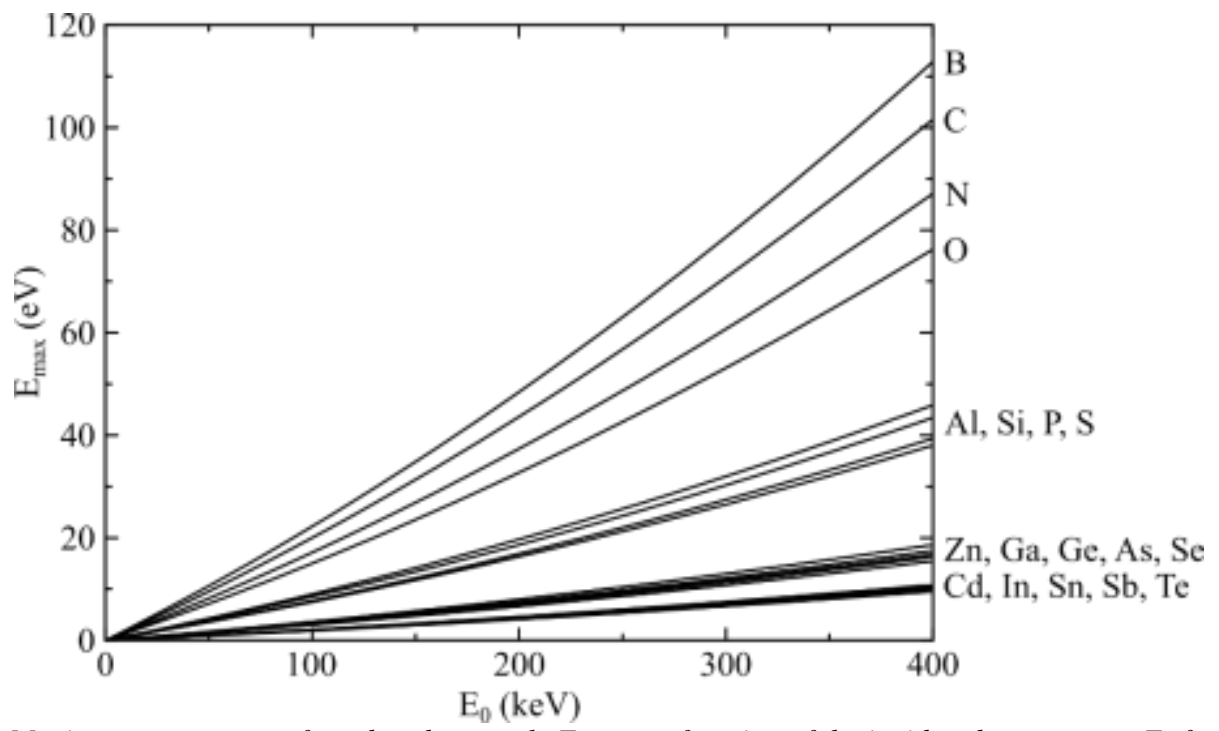

Figure 20: Maximum energy transferred to the sample $E_{\max }$ as a function of the incident beam energy $E_{0}$ for a range of common atoms in semiconductors.

Atomic displacement can also happen through sputtering. Beam sputtering involves atoms close to the sample surface so that a displaced nucleus can be ejected from the sample. Given that the nucleus does not have to be relocated to an interstitial site in the crystal, this mechanism requires much less energy than atomic displacement into the "bulk" crystal. In such a case, the displacement energy $E_{d}$ can be expressed as a function of the sublimation (i.e. transition from the solid phase to the gas phase) energy $\mathrm{E}_{\text {sub }}$ of the material. Egerton et al. [86] showed that a reasonable approximation of $\mathrm{E}_{\mathrm{d}} \mathrm{can}$ be given by $E_{d}=\frac{5}{3} E_{s u b}$. Given that the sublimation energy of most materials is of the order of a few eV, it appears quite likely that most samples are susceptible to beam sputtering in the TEM. Sputtering will need to be particularly carefully considered in TEM studies of the emerging 2D materials.

In both cases - atomic displacement and beam sputtering - one can see that it is possible to avoid electron beam damage by reducing the acceleration voltage of the microscope below the knock-on voltage. If this is not possible - if the gun does not allow such a low voltage, or if the lower voltage results in a poor image contrast - one can try to reduce the radiation dose by limiting the specimen exposure time. Another potential solution, assuming that beam sputtering is the dominant beam damage mechanism, would be to coat the exit surface of the TEM foil with a heavy element [84].

\section{Sample preparation}

In order to conduct a TEM experiment, the sample has to meet two key requirements: the sample must be electron transparent and the sample must fit in the TEM sample holder. These conditions imply that the sample must be thinner than $200 \mathrm{~nm}$ approximately and that the sample must be mounted on top of a $3.05 \mathrm{~mm}$ diameter ring. Therefore, one of the important parts of a TEM experiment is sample preparation. Different methods exist for preparing samples (mechanical polishing, focussed ion beam (FIB), electropolishing, chemical etching, ultramicrotome, etc.) depending on the material properties. The most commonly applied in studies of semiconductor defects are mechanical polishing and FIB. Box 1 outlines a typical procedure for sample preparation by mechanical polishing, with some tips to help ensure successful results.

- Using a diamond scribe and/or a diamond saw, cut two rectangles of typical size $3 \mathrm{~mm} \times$ $1.5 \mathrm{~mm}$ from the original sample.

Tip: Cut along specific crystallographic directions - this will facilitate finding the zone-axis during the TEM experiment.

- Stick the top surfaces of the two rectangles to each other using an epoxy resin.

Tips: (1) Make the glue line as thin as possible to avoid damaging the front surface during the ion milling step. (2) Make sure that the sample can sit on a support stub on its own with the glue line perpendicular to the support. This 
ensures that the electron beam will be almost parallel to a zone-axis once in the

TEM (some microscopes do not allow for large sample tilts).

- Mount the sample on a stub using thermoplastic wax.

- Grind the first side of the sample using SiC or diamond abrasive discs until a flat surface is obtained (Take special care to avoid any chipping near the glue line).

Tips: (1) Gradually decrease the grain size of the disc. (2) Make sure that for a given disk the sample thickness does not fall below $\sim 2-3$ times the grain size of the disk - this will ensure that there will be no damage from grinding in the final foil.

- Polish the first side of the sample using a cotton wheel in a dimpler until no scratches are visible in an optical microscope.

- Flip the sample and repeat the grinding/polishing procedure until the sample thickness reaches $20-40 \mu \mathrm{m}$ approximately.

Tips: Use a P400 disc (average grain $35 \mu \mathrm{m}$ ) down to a sample thickness of 80$100 \mu \mathrm{m}$, then P600 (average grain $25.8 \mu \mathrm{m}$ ) down to 50-70 $\mu \mathrm{m}$ and finally P800 (average grain $21.8 \mu \mathrm{m}$ ) down to $40-60 \mu \mathrm{m}$. Final polishing down to $20-40 \mu \mathrm{m}$ is made with a cotton wheel in the dimpler.

- Stick a $3.05 \mathrm{~mm}$ TEM ring onto the sample.

- Mill the sample using an ion milling system until a hole appears in the sample. The ion milling system uses two $\mathrm{Ar}^{+}$ion guns operating between 0.1 and $6 \mathrm{kV}$ accelerating voltage with an incident angle up to $\pm 10^{\circ}$.

Tips: (1) Position the sample with the glue line on the rotation axis of the ion mill stage. (2) Operate the system at 5-6 kV until colourful fringes start appearing around the glue line, then decrease the voltage down to $3 \mathrm{kV}$ until a hole forms near the glue line, finally, decrease the voltage down to $0.1-2 \mathrm{kV}$ for 15 minutes to clean the surface from high energy ion mill damage.

Box 1: Typical procedure for preparation of TEM samples by mechanical polishing, with some tips and tricks.

Preparation by FIB involves putting the sample in an SEM equipped with an electron gun and a $\mathrm{Ga}^{3+}$ ion gun, as well as a source for Pt deposition - other elements (often C,W) can also be used. The electron gun is usually operated at fairly low voltage $(5 \mathrm{kV})$ and is used for imaging, while the ion gun operates at much higher voltage (often $30 \mathrm{kV}$ ) and is used for milling. Imaging with the ion gun is also possible but one has to minimize the exposure to the beam as it damages the sample (as could be expected from a beam whose primary function is to mill the sample!). In essence, FIB aims to excavate a thin slice out of the bulk sample, and a typical procedure, with some helpful tips, is given in Box 2.

- Deposit a protective Pt layer on the surface of the sample in order to protect it from the high energy ion beam.

Tip: To minimize damage, first deposit Pt using the electron gun, then with the ion gun.

- Using the ion gun for removing material, and the electron gun for monitoring, mill two ditches, one on each side of the Pt layer.

- Now that the sides of the sample slice are exposed, make a cut underneath - this will separate the slice from the bulk sample.

- Extract the slice from the bulk sample using a sharp needle-shape probe, and transfer the sample to a TEM grid.

- Gradually thin down the sample slice from both sides using ion beam of decreasing currents (in order to remove previous beam damage) until electron transparency thickness $(\sim 200 \mathrm{~nm})$.

Tip: For best results (to make a sample suitable for atomic scale imaging), perform a final cleaning of the sample using the ion gun at a lower voltage, or using a separate ion milling system (such as the system mentioned for mechanical preparation in Box 1) at $0.1-1 \mathrm{kV}$ 


\section{TEM equipment}

Although the exact architecture of a TEM instrument will depend on its mode of operation (e.g. parallel beam, convergent beam), most TEMs are built with a common arrangement. Figure 21 presents the basic architecture for a TEM operating in parallel beam illumination, and each component is described below.

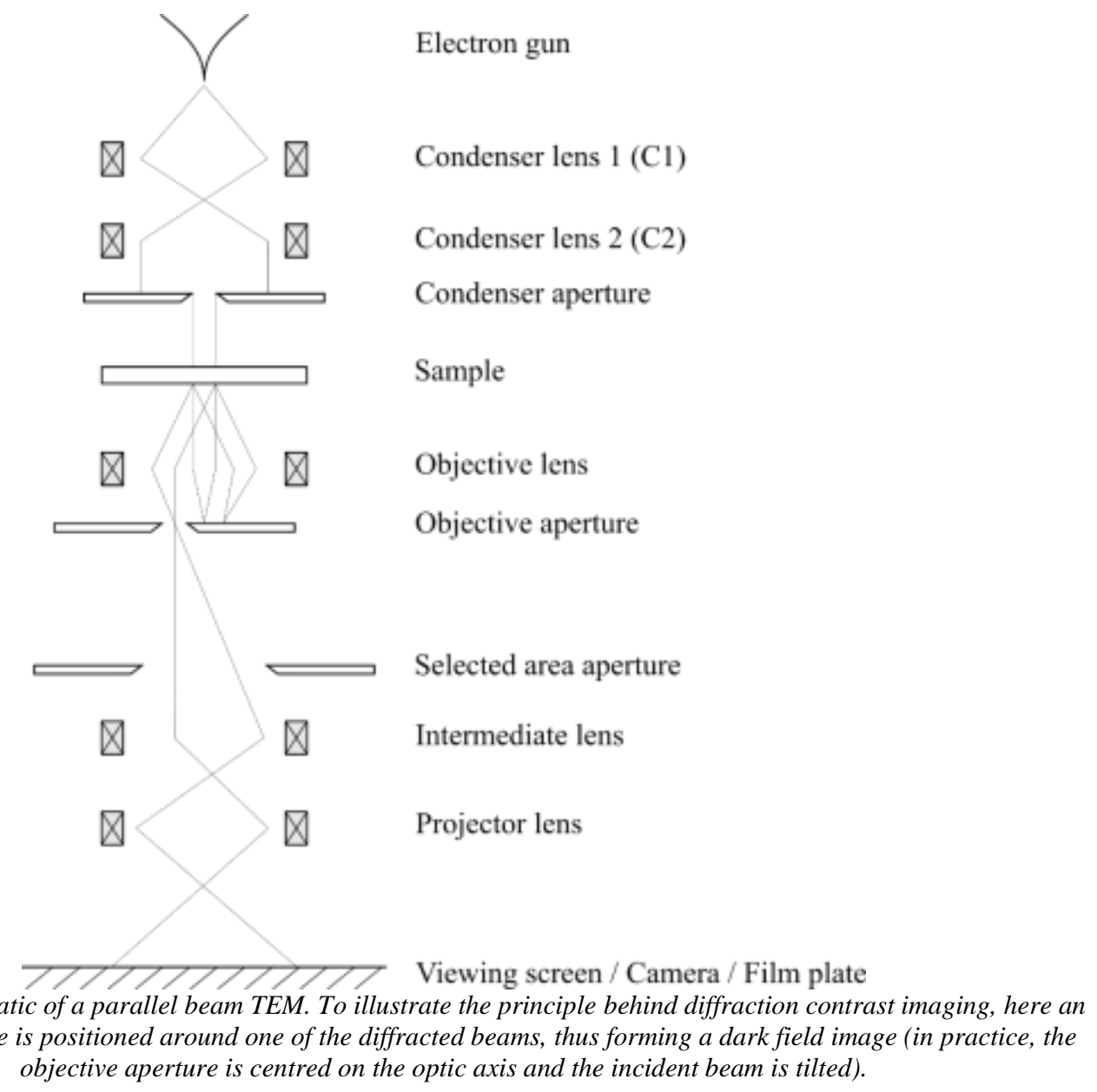

Electron gun: The most common types of electron guns in recent TEMs are the $\mathrm{LaB}_{6}$ filament and the field emission gun (FEG). Although the emission mechanisms are different for these two types of guns - thermionic $\left(\mathrm{LaB}_{6}\right)$ or field emission (FEG) - the main idea is that an electron source is placed at the cathode with respect to two anodes (or Wehnelt (i.e. a 'grid' which acts as an electrostatic lens) plus anode in the case of a thermionic gun). The electrons are then extracted from the source (cathode) and accelerated by the anode to the operating voltage of the microscope. An undeniable advantage of FEGs over $\mathrm{LaB}_{6}$ filaments is that electrons emanate from a much smaller region of the cathode, leading to a smaller, brighter, and more coherent source.

Condenser lens $1(\mathrm{Cl})$ : The first condenser lens is used to demagnify (or magnify in the case of a FEG) the electron source.

Condenser lens 2 (C2): The second condenser lens is important in that it determines the area of exposure of the specimen. By underfocussing $\mathrm{C} 2$, the sample is illuminated by a quasi-parallel beam. By focussing $\mathrm{C} 2$, the sample is illuminated by a convergent beam (in some microscopes, a lens called the 'upper objective lens' is inserted between $\mathrm{C} 2$ and the sample to further decrease the convergence angle). A parallel incident beam is used to achieve a coherent electron beam - which gives rise to sharp diffraction patterns. When the electron beam is made convergent, the electron probe can be rastered across the surface (hence the appellation scanning TEM or STEM) with a resolution close to the probe size (related to the convergence angle). In aberration-corrected microscopes, the $\mathrm{C} 2$ lens is followed by aberration 
correctors, which are essentially a set of additional multipole lenses whose functionality is to correct a specific type of aberration - e.g. astigmatism, spherical aberration, chromatic aberrations.

Condenser aperture: Along with the second condenser lens, an aperture can be inserted in order to control the illumination of the specimen as well as the convergence angle of the beam.

Sample: The sample is mounted in the sample holder which can be translated in the three spatial directions and tilted around the two axes perpendicular to the optic axis.

Objective lens: An objective lens is placed after the sample in such a way that the sample is in the focal plane of the lens. The distance between the sample and the objective lens is called 'eucentric height'.

Objective aperture: The objective aperture is located in the focal plane of the objective lens. Consequently, the objective aperture is placed where the diffraction pattern of the sample forms. This aperture is used to select a particular set of diffraction conditions (and therefore a particular set of information about the sample), thus enabling particular imaging conditions which will be described later. Selected area aperture: The selective area aperture is placed in the image plane of the objective lens. It allows one to virtually select which part of the sample is illuminated.

Intermediate and projector lenses: The intermediate and projector lenses are used to select which mode, between imaging and diffraction, will be observed on the viewing screen. When imaging mode is selected, the lenses are adjusted so that the viewing screen is in the image plane of the combination of the two lenses. Similarly, when diffraction mode is selected, the lenses are adjusted so that the viewing screen is in the focal plane of the combination of the two lenses. Using two lenses enables the magnification of the image or diffraction pattern.

Viewing screen: The electrons thus selected can finally hit the phosphor screen leading to observable fluorescence. To record an image, the viewing screen can be lifted to allow the beam to hit a photographic film or a camera.

\subsubsection{Technique for diffraction contrast imaging of defects - Bright Field, Dark Field, Weak Beam Dark Field}

Diffraction contrast imaging is achieved by viewing the diffraction pattern of the specimen and using the objective aperture to select only one particular diffraction spot. Hence only the planes diffracting the beam in this particular way will appear on the viewing screen. Depending on the beam going through the objective aperture (either the direct beam or a scattered beam) and on the sample orientation allowing the excitation of different diffraction conditions. This type of imaging is qualified as bright field imaging, dark field imaging or weak beam dark field imaging and is mainly used for the characterisation of defects as illustrated in Figure 22. The application of this technique to the characterisation of dislocations will be further discussed in Section 10.3.1.4. In theory, diffraction contrast imaging is possible regardless of the sample orientation, as long as a diffraction pattern can be obtained. In practice, the contrast of the image can be strongly increased if the sample is tilted away from the zone-axis, along the Kikuchi band associated with the selected reflection. This results in the sample being in 'two-beam conditions', where only the direct beam and one diffracted beam are strongly excited. It is also recommended to select a deviation parameter $\boldsymbol{s}>0$ in order to further improve the contrast. ( $\boldsymbol{s}$ is also called the excitation error and describes, in the reciprocal space, how far away the system is from the Bragg conditions). 

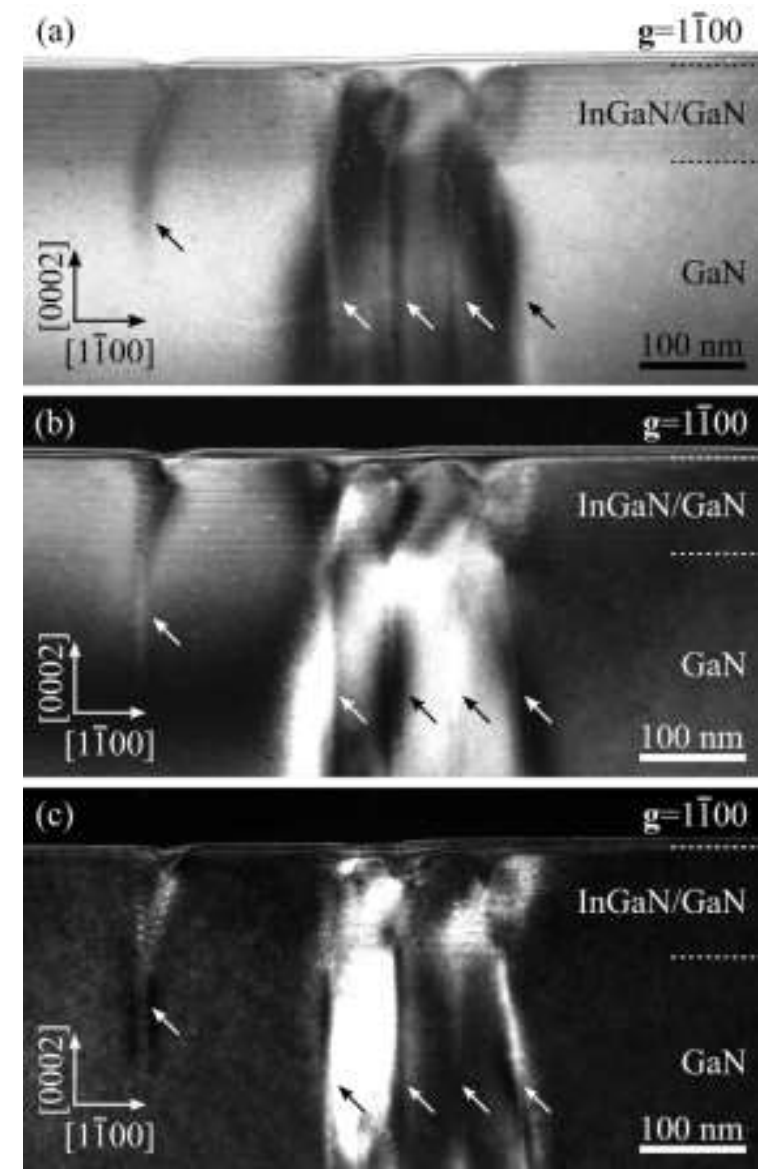

Figure 22: Bright field (a), dark field (b) and weak beam dark field (c) TEM images of an InGaN/GaN quantum well structure observed along the $\langle 11 \overline{2} 0\rangle$ zone-axis using $\boldsymbol{g}=1 \overline{1} 00$. Dislocations in each images are indicated by an arrow.

Bright field imaging is obtained when an objective aperture is used to select only the direct beam. Given that the direct beam contains the information about the planes which do not diffract the incident beam, all the material which does not diffract will appear bright, whilst regions of material where the beam is scattered (e.g. defects) will appear dark (Figure 22(a)).

Dark field imaging is obtained when an objective aperture is used to select a diffracted beam. One issue with dark field imaging as can be observed in Figure 21 is that the selected beam is off-axis and therefore subject to aberrations by the intermediate and projector lenses. In most TEMs this is solved by tilting the incident beam adequately so that the diffracted beam is kept on-axis. When the sample is in twobeam conditions, the majority of electrons not contributing to the direct beam are contributing to this particular diffracted beam. Therefore a dark field picture results in the negative of a bright field picture, with all the diffracting planes appearing brighter (Figure 22(b)).

Weak beam dark field imaging occurs when the same conditions as for dark field are fulfilled, but the sample is tilted slightly further (perpendicularly to the Kikuchi band) to increase the deviation parameter $\mathbf{s}$. In that case, the diffracted beam $\mathbf{g}$ becomes weak, and the higher order diffraction spots $3 \mathbf{g}$ (or $5 \mathbf{g}$ ) becomes stronger. Like in dark field, an objective aperture is put around $\mathbf{g}$. The resulting image is similar to a dark field picture except that only the planes which are strongly diffracting appear (Figure 22(c)). This technique allows us to have a better resolution: when observing dislocations for example, only the planes closest to the core will appear. Therefore, dislocations appear much thinner, i.e. dislocation densities in high dislocation density films can be counted more easily and their Burgers vectors found.

\subsubsection{Techniques for compositional analysis near defects - High angle annular dark field STEM, EDX}

High angle annular dark field STEM (STEM-HAADF)

We mentioned earlier that high angle scattering (> $50 \mathrm{mrad}$ ) of the electron beam by the nuclei of atoms is dictated by Rutherford scattering, with the cross section proportional to $\mathrm{Z}^{2}$. STEM-HAADF is built around this effect. By using an eponymous annular detector that collects electrons scattered at a high angle, a Z-contrast image is generated as the convergent probe is scanned across the sample. The term dark field originates from the fact that only diffracted electrons contribute to the image. STEM-HAADF 
is therefore suited for the study of compositional variations across the sample, where regions of higher average atomic number appear brighter than regions of lower average atomic number (as illustrated by the bright contrast from InGaN quantum wells in a GaN matrix in Figure 23). However, STEM-HAADF cannot be straightforwardly used to quantify the composition. Other techniques (e.g. EDX) often embedded in the same microscope are usually used for this purpose. One also has to bear in mind that the thickness of the TEM foil will affect the image contrast - thinner regions appear darker than thicker regions - and that strain can also influence the contrast if the detector does not collect solely electrons diffracted at a sufficiently large angle (illustrated by contrast arising at a dislocation in Figure 23).

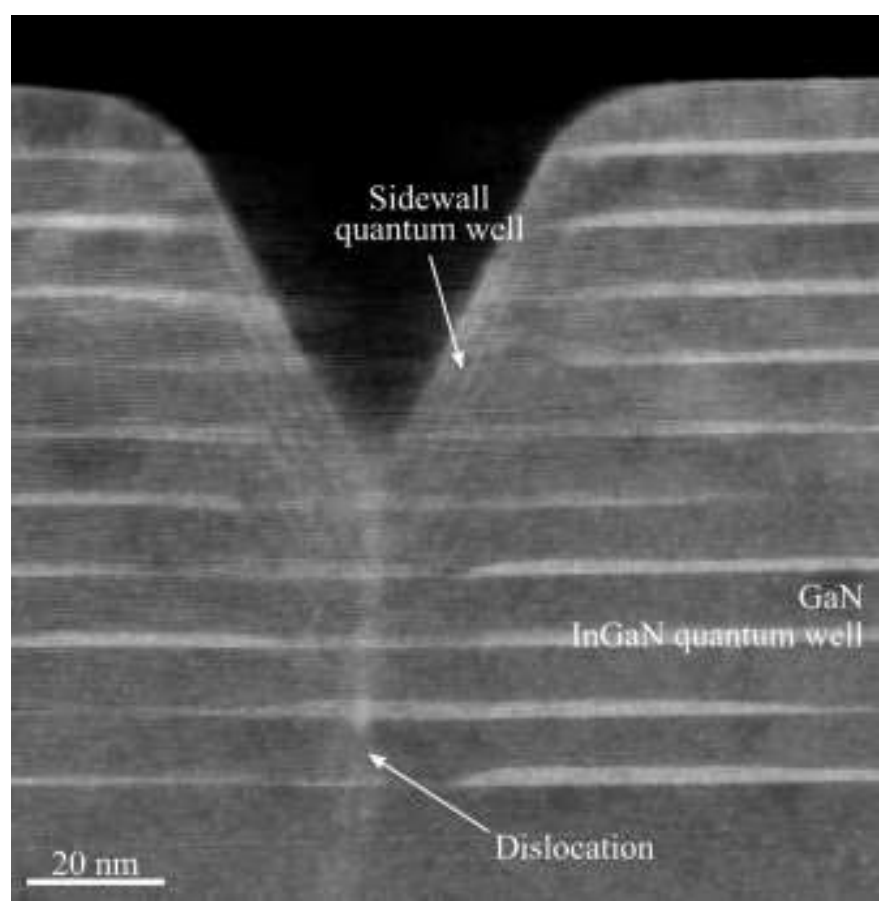

Figure 23: STEM-HAADF image of a dislocation in an InGaN/GaN quantum well sample. The quantum wells appear brighter due to the greater atomic number of InGaN over GaN. Quantum wells can also be seen forming on the sidewalls of the pit formed at the termination of the dislocation. The dislocation is also visible indicating strain-related contrast.

\section{Energy dispersive $X$-ray spectroscopy $(E D X)$}

Unlike STEM-HAADF which provides essentially qualitative compositional information, EDX is in essence quantitative. Provided that sufficiently high signal can be detected, the atomic species present in the sample and their fraction, can be determined. The atomistic mechanism for generation of the EDX signal is explained in Section 10.2.2.6. Here we add some comments about typical quantification routes used in TEM. Quantification of the proportion of each species can be obtained by analysis of the intensity of the peaks relating to different elements in the X-ray spectrum. Most TEM analysis software employs the Cliff-Lorimer method [87]. EDX can therefore be a useful technique to investigate compositional fluctuations at defects, for example, in order to investigate segregation phenomena. However, a common mistake is to overestimate the capabilities of the technique. For example, as we explicitly mentioned, the signal needs to be sufficiently high to be able to extract information from a spectrum, which can be more difficult to achieve when the signal arises from a small volume in a thin TEM foil. Although the systems are continuously being improved, minute amounts of impurities in the material, as is the case for semiconductor dopants, will not be detected. Unfortunately, trying to increase the EDX signal often results in an increase of the electron dose in the sample, which can damage the sample. Furthermore, the Cliff-Lorimer analysis does rely on a calibration factor, termed the CliffLorimer factor, which can be derived from standard samples, but which can vary with the foil thickness and electron beam energy among other factors. This can limit the accuracy of the quantification unless treated with great care. 


\subsubsection{Techniques for high-resolution imaging of defects - High resolution TEM, STEM High resolution TEM}

In the previous section, we have seen that diffraction contrast arises from selecting a single diffraction spot using the objective aperture. Thus only the information conveyed by the selected diffraction condition - a specific set of planes in Bragg condition - will contribute to the image. However, if one uses a larger objective aperture, or no aperture at all, several diffraction spots can contribute to the image. For example, in a configuration where two $(\mathbf{0 g}$ and $\mathbf{g})$ or three beams $(-\mathbf{g}, \mathbf{0 g}$ and $\mathbf{g})$ are allowed through the aperture, one can visualize on the viewing screen a set of black and white fringes resulting from the interference of these beams. Although these fringes cannot be directly interpreted as atomic planes (those that relate to the $\mathbf{g}$ vector), the fringe spacing relates to the plane spacing. If more diffraction spots are selected, then the superposition of interference fringes from several $\mathbf{g}$ vectors results in interference 'spots', that one can relate to the crystal structure of the sample. High resolution TEM (HR-TEM) is obtained when the microscope is set up in order to make these interferences visible on the screen. Let us emphasize this again: although it can be very tempting to associate atoms with the interference spots on the screen, one must always be aware that this is technically incorrect. Moreover, the image displayed on the viewing screen is a convolution of the interference pattern and of the contrast transfer function of the objective lens (i.e. the ability of the objective lens to transfer certain spatial frequencies). This contrast transfer function is far from being constant for all frequencies, and can dramatically affect the image contrast just by changing the focus of the microscope. As a general rule of thumb, HR-TEM should mainly be used qualitatively, typically to observe the undefected crystal or identify the presence of a defect. For more quantitative interpretations such as identifying the atomic arrangement at the core of a dislocation, one must compare the experimental image with simulations. An additional interesting analysis technique in HR-TEM is geometric phase analysis [88], which allows one to map the distribution of strain across the image - for example to identify the tensile and compressive regions of a dislocation.

\section{High resolution STEM}

With the advent of aberration correctors, high resolution STEM (HR-STEM) is perhaps currently the best suited technique for the study of defects at the atomic scale. HR-STEM is based on the same idea as STEM-HAADF where the contrast is due to Rutherford scattering - i.e. proportional to $Z^{2}$. The main difference is that aberration correctors allow for smaller probe size and therefore improved resolution, down to the atomic scale. A crucial difference with the previous technique (HR-TEM) is that in HRSTEM the images can be directly interpreted in terms of atoms. You still have to bear in mind that the typical TEM sample is not atomically thin, hence an 'atom' in the image correspond in fact to an atomic column. This is therefore the technique of choice to study atomic arrangement around defects (See Figure 24).

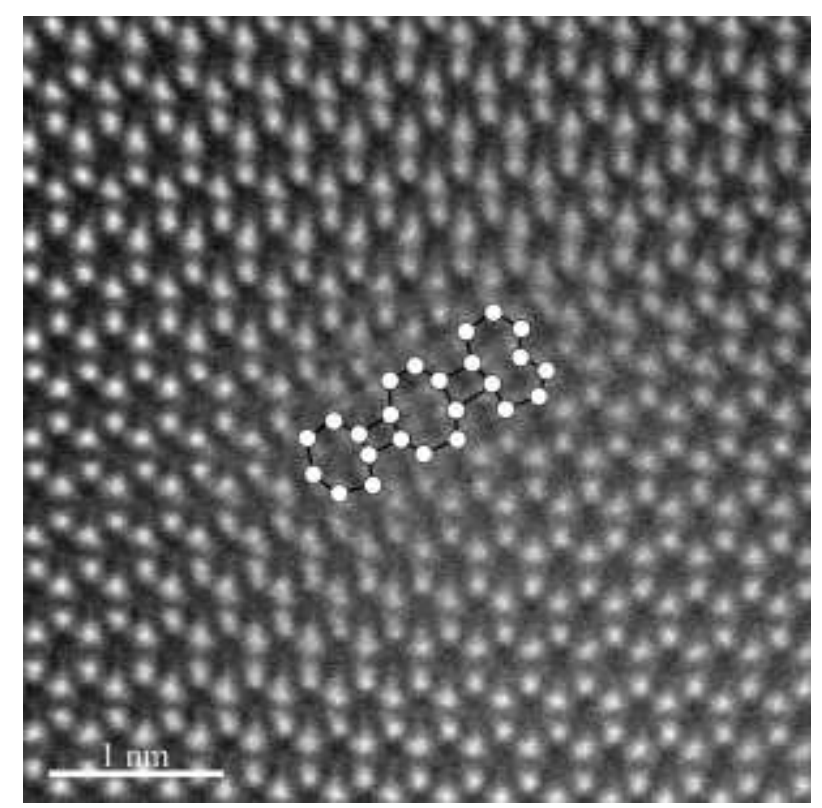

Figure 24: HR-STEM image of the core structure (here double 7-4-8-4-9 atom rings) of a mixed dislocation in AlGaN observed along [0001]. 


\title{
10.3 Examples of the application of microscopy to semiconductor materials
}

\author{
10.3.1 Dislocation densities and Burgers vectors in GaN \\ 10.3.1.1 Motivation for the application of microscopy to study dislocation types and \\ densities in GaN
}

The nitride family of materials is perhaps unique amongst the conventional compound semiconductors in the extent to which nitride devices are robust to the presence of high densities of extended defects, particularly dislocations. This allows the use of heteroepitaxial layers grown on highly mismatched substrates in the fabrication of both electronic and optoelectronic devices in GaN, with dislocation densities in working light emitting diodes (LED) and high electron mobility transistors (HEMTs) frequently in excess of $10^{9} \mathrm{~cm}^{-2}$. However, dislocations do impact the performance of nitride devices, particularly laser diodes [89], and this has provided an impetus for the development of techniques both for the reduction of dislocation densities in heteroepitaxial materials and for the growth of bulk GaN with dislocation densities down to $\sim 10^{4} \mathrm{~cm}^{-2}$ [90]. Provision of feedback for the development of the materials growth has thus required the availability of techniques which allow quantification of dislocation densities ranging over five orders of magnitude. In typical (0001) GaN heteroepitaxial layers, the basis of most devices, three types of threading dislocations are observed: pure edge, or ' $\mathrm{a}$ type' (Burgers vector, $\mathbf{b}=1 / 3<11-20>$ ), pure screw, or 'c-type' $(\mathbf{b}=<0001>$ ), and mixed, or 'c $+a$ type' $(\mathbf{b}=1 / 3<11-23\rangle)$. Quantification of the proportion of dislocations of each type can aid understanding of the mechanisms of both dislocation generation and reduction. Information on these mechanisms can also be deduced from the spatial distribution of dislocations across the surface of a sample. Microscopy provides the means to access all of these parameters of the dislocation distribution in the nitrides, but different methods are more appropriate to different dislocation density regimes.

\subsubsection{AFM methods for dislocation density quantification}

As was mentioned in Section 10.2.1.1, the termination of a dislocation at a sample surface necessarily results in the formation of a small surface pit, to achieve an equilibrium of forces between the dislocation line tension and the surface tension. The formation of such pits or "dimples" was predicted by Frank as early as 1951 [91], who also noted that dislocations with a screw component intersecting a close-packed surface must be associated with a step edge emanating from the dislocation termination. As grown $\mathrm{GaN}$ surfaces exhibit dislocation pits associated with all types of threading dislocations, and step edge terminations are indeed seen at screw and mixed dislocations. This allows AFM imaging of the dislocations and discrimination between dislocations with and without a screw component. However, the relevant pits are small in lateral extent (typically 20-30 nm for mixed dislocations and less than 10 $\mathrm{nm}$ for edge dislocations) and also shallow (less than a nanometre). Pits associated with edge dislocations in particular are often only visible when tip sharpness is carefully preserved. (Whilst in general blunt tips limit the lateral resolution rather than the vertical resolution, a blunt tip cannot penetrate into a narrow pit, or may not penetrate its full depth. In the latter case, the ability to detect the dislocation depends on the relative magnitudes of the depth to which the tip penetrates and the background noise). A variety of wet etching methods have been applied to increase the size of dislocation-related pits at GaN surfaces (and are reviewed in Reference [92]), but in many cases the resulting pits are sufficiently large that for samples with dislocations densities in excess of $10^{8} \mathrm{~cm}^{-2}$ (which includes most common materials for LEDs and HEMTs), pits tend to merge making detailed quantification difficult.

A more successful approach for (0001)-oriented $\mathrm{GaN}$ grown by the most common technique, metalorganic vapour phase epitaxy (MOVPE), is the application of a surface treatment using $\mathrm{SiH}_{4}$ and $\mathrm{NH}_{3}$ in the deposition system immediately post-growth. This can be straightforwardly used to increase the diameters of pits associated with edge and mixed dislocations to approximately $45 \mathrm{~nm}$ and $60 \mathrm{~nm}$ respectively, allowing them to be easily imaged in AFM [92]. Tapping mode images of a sample before and after surface treatment are shown in Figure 25. Edge dislocations may be distinguished from mixed dislocations not only by the smaller pit size but also because the treatment leaves terraces 

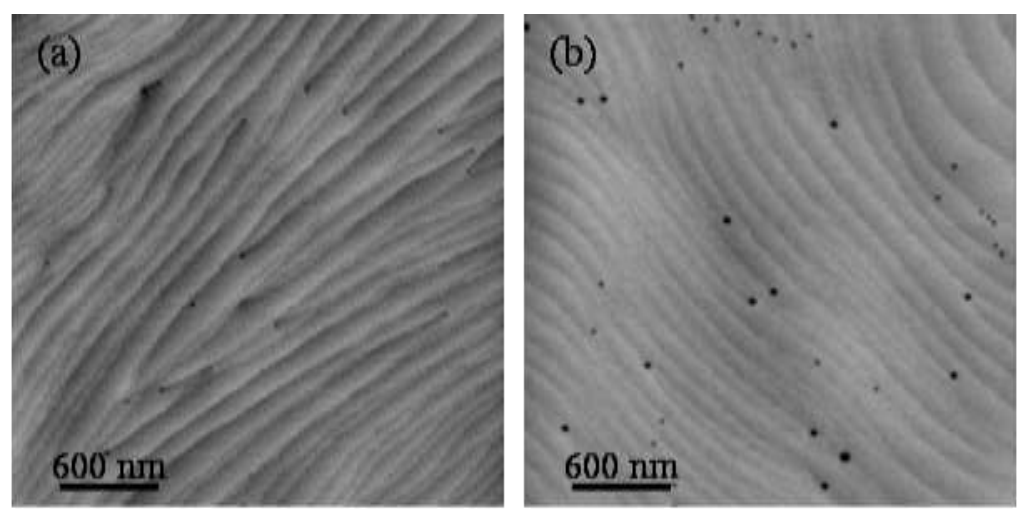

Figure 25: Images of a (0001) GaN surface before (a) and after (b) treatment with SiH4 and $\mathrm{NH}_{3}$ to increase the size of the dislocation pits. Reprinted from Reference [92] with permission from Elsevier.

distinguishable at the surface so that step terminations at mixed dislocations can be identified. The identification of pure screw dislocations by this method has not been thoroughly explored since most MOVPE-grown materials contain very few pure screw dislocations. This method has been applied to samples with dislocation densities ranging from $\sim 10^{5} \mathrm{~cm}^{-2}$ [93] to $5 \times 10^{9} \mathrm{~cm}^{-2}$ [94], although for the lowest dislocation densities it is very slow, since imaging must be carried out at a sufficient resolution to locate the small pits over very large areas. It has been extensively used to explore spatial distributions of dislocations in $\mathrm{GaN}$ [95] and to relate them to defect formation mechanisms. It has also been used to locate dislocations terminating at the surface of large (few micron height) three-dimensional islands, including on semi-polar facets (facets inclines to (0001)) [92]. It has been used on films grown in nonpolar orientations [96], and has in this context been suggested to have some efficacy for the identification of stacking faults as well as dislocations. However, it should be noted that the specific surface treatment outlined here is not generalizable to other semiconductors, and indeed fails on strained AlGaN surfaces, although it is effective on relaxed $\mathrm{AlGaN}$ [97]. Nonetheless, it provides a useful example of the level of detail which basic topographic AFM can provide in materials where sufficient surface topography exists or can be generated at the surface terminations of extended defects.

\subsubsection{SEM methods for dislocation density quantification}

One of the advantages of ECCI in the SEM is the ability to acquire images rapidly (in the order of minutes) from large areas of the surface of a sample (of order $10 \mu \mathrm{m} \times 10 \mu \mathrm{m}$ ). For dislocations which thread through the sample and reach the surface, dislocation densities can be determined by simply counting the number of dislocations in the image. There are a number of different techniques for obtaining a count of the dislocations ranging from a simple manual count, to the use of appropriate plugins in image processing packages such as ImageJ [98], to the development of bespoke image processing software exploiting machine learning techniques. If the diffraction condition(s) can be determined, it is in principle also possible to identify the dislocation type as described in Section 10.2.2.2. Using the procedure described in Section 10.2.2.2, Naresh-Kumar et al. [99] identified 300 threading dislocations in a GaN thin film. Approximately $51 \%$ were identified as pure edge dislocations (Burgers vectors $1 / 3<11-20\rangle$ ), $\approx 42 \%$ were mixed dislocations (Burgers vectors $1 / 3<11-23>$ ), and pure screw dislocations accounted for $\approx 7 \%$ of the total dislocation density (Burgers vectors $<0001>$ ).

While ECCI is an excellent technique for quantification of defects which reach the surface, it is harder to quantify dislocations below the surface due to the surface sensitivity of the ECCI technique and the difficulty in determining the precise sampling depth of the technique. However, it is possible to obtain quantitative data on dislocations which lie below the surface, as illustrated for example by the paper by Carnevale et al. [100] on misfit dislocations in GaP lying at up to around $100 \mathrm{~nm}$ from the surface.

The ability to image large areas of a sample makes it possible to probe the statistical distribution of the dislocations threading to the surface of a sample. Ripley's $K$-function $[101,102]$ is one of the most widely used methods to analyse point pattern data providing information on the variation of the density of points under investigation and it determines whether they are clustered (under-dispersed), random (dispersed) or regular (over-dispersed).

Ripley's function produces concentric circles with increasing radii around each identified point in an image and counts the number of neighbouring points that are contained within each circle. This process is repeated for each point within the image with the cumulative total for a given radius $r$ computed over 
a range of values of $r$ to produce the distribution function $K(r) . L(r)$ is a function derived from $K(r)$ which is easier to interpret, and is simply the deviation between the measured $K(r)$ and a points distribution of the same density but exhibiting complete spatial randomness (CSR)[103].

To determine the statistical significance of any deviation from CSR, any deviation can be evaluated using simulated significance intervals by computing the lowest and highest value of $L(r)$ for each radius $r$ to define the lower and upper bounds of a significance envelope. A Monte-Carlo method is generally employed for these simulations. If the empirical $L$-function lies above the upper bound of the CSR envelope, this implies that there is a higher density of points than would be expected under CSR and there is clustering. Similarly, if the empirical $L$-function lies below the lower bound, this indicates that there is a lower density of points than would expected under CSR, and thus the points are over-dispersed. Figure 26(a) shows an ECCI micrograph from a GaN thin film where the dislocations are highlighted by red circles. This point pattern has then been analysed using $L(r)$ and this is shown in Figure 26(b). The deviation of experimental $L(r)$ outside the $90 \%$ significance envelope indicates a statistically significant over dispersion of dislocation for a radius of $\lesssim 150 \mathrm{~nm}$; this is likely due to the mutual repulsion of neighbouring dislocations due to their associated strain fields $[104,105]$ and to limits imposed by spatial resolution which is $\approx 70 \mu \mathrm{m}$ for this image. There is also statistically significant clustering of dislocations for a radius of $\gtrsim 500 \mathrm{~nm}$. Examination of Figure 26 shows that the dislocations are arranged in long lines, in particular along boundaries between regions exhibiting different grey scales in the image. The changes in grey scale indicate changes in orientation between subgrains in the film. The clustering indicated by the $L(r)$ is probably due to these lines of dislocations.

For more information on statistical analysis of the distribution of dislocations the reader is referred to the work of Moram et al. who have published detailed studies on the use of spatial analysis techniques for the analysis of defects observed in AFM and CL images, see for example [105,106]. Note that Moram et al. employed the AFM techniques for dislocation identification described in Section 10.3.1.2.
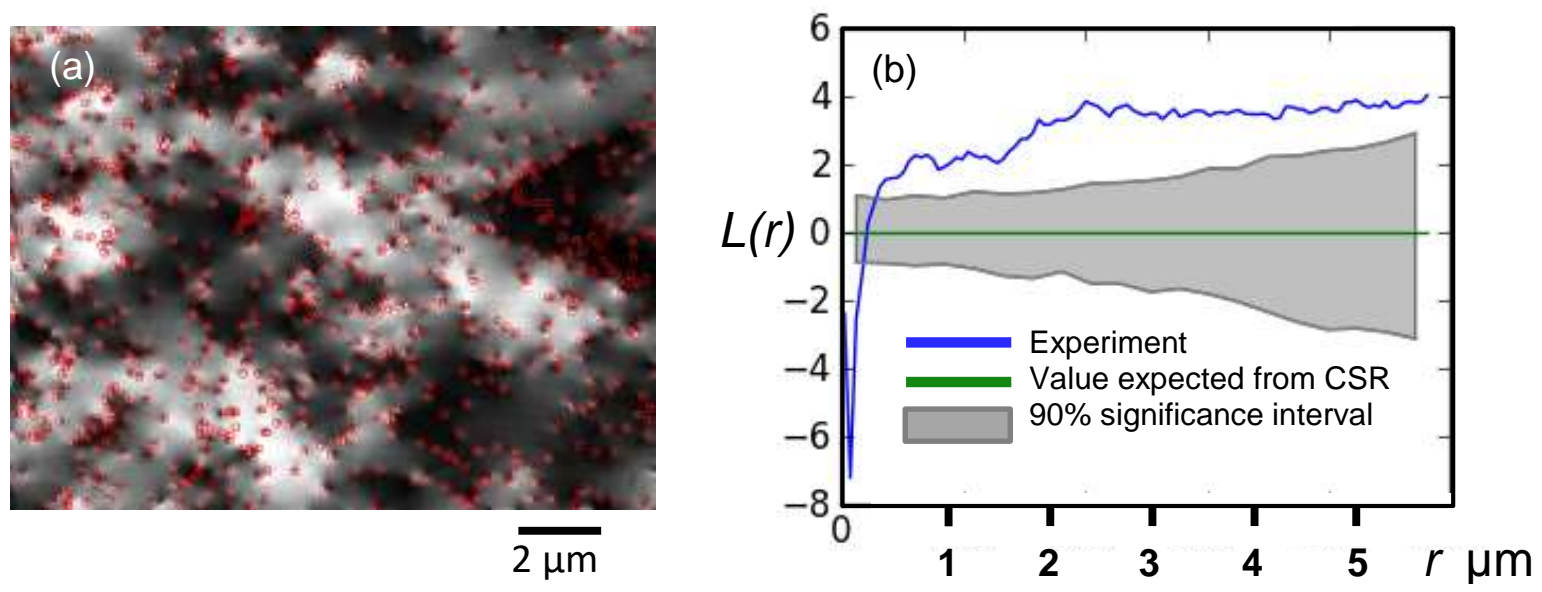

Figure 26: (a) ECCI micrograph from a GaN thin film with dislocations highlighted with red circles $(b) L(r)$ for the dislocation distribution shown in $(a)$, showing the $L(r)$ determined from experiment, $L(r)$ expected from complete spatial randomness $(C S R=1)$ and a significance interval of $90 \%$ (Dislocations identified and statistical analysis undertaken using in-house software developed by Simon Kraeusel). Adapted and reprinted from [107] with permission from Cambridge University Press.

\subsubsection{TEM methods for dislocation density quantitation}

In Section 10.2.3.2 we saw that dislocations can be observed using TEM, and we will discuss this in more detail shortly. Probably the most 'accurate' way to obtain the density of dislocations is by using plan-view imaging. In $\mathrm{GaN}$ devices, the dislocations that matter the most are often those which thread toward the surface. These will be seen end-on in a plan-view geometry. The density of dislocations will therefore be obtained by directly counting how many dislocations intersect the foil over a given area. Here the word 'accurate' above was purposely written with quotation marks because the area of material is relatively small (typically less than $1 \mu \mathrm{m}^{2}$ ), making the density thus obtained statistically less reliable 
than if it were obtained with other techniques (e.g. AFM or SEM). Nevertheless its reliability is still typically better than density values obtained from cross-sectional TEM observation.

However, cross-sectional imaging can provide a density of dislocation, but over a much smaller area of materials hence reducing the statistical strength of the measurement. Moreover, in this geometry most dislocations will run parallel to the TEM foil. Therefore, in order to obtain a density of dislocations in cross section, one has to count the number of dislocations at a certain position in the foil, but the thickness of the TEM foil is also required. This last value can be obtained through additional TEM analysis, typically by comparing an experimental convergent beam electron diffraction (CBED) pattern with a simulated one. EELS can also be used for this purpose, by comparing the zero-loss peak with the total intensity of the low-loss spectrum. As such, cross-sectional TEM imaging is not the ideal technique to obtain a reliable density of dislocations. The real strength of the approach is that one can visualize the behaviour of dislocations throughout the thickness of the film - which is information that cannot be obtained by plan-view imaging or any surface techniques. For example, in Figure 27, we can see that a high density of dislocations is generated near the substrate/film interface (bottom of the picture), but also we see that the dislocations interact with each other within ca. $2 \mu \mathrm{m}$ from the interface, hence reducing greatly the number of dislocations that thread to the surface (top of the image).

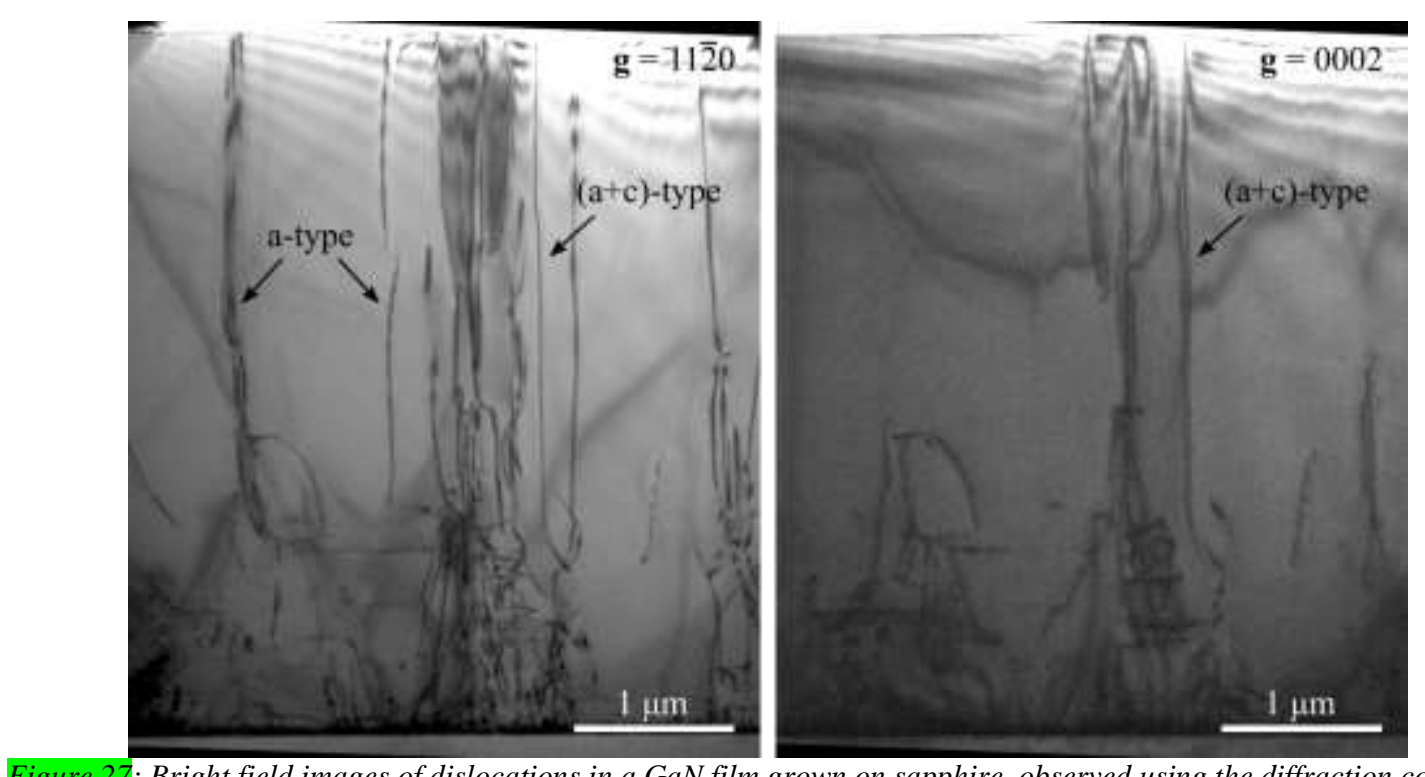

Figure 27: Bright field images of dislocations in a GaN film grown on sapphire, observed using the diffraction conditions $\boldsymbol{g}=11 \overline{2} 0$ (left) and $\boldsymbol{g}=0002$ (right).

\section{g.b analysis}

Let us consider further why dislocations generate contrast in the TEM: we will see that not only can dislocations be imaged in the TEM, but that the technique also allows one to determine the Burgers vector of the dislocations. A coherent electron beam will be diffracted by the crystal lattice. However, a dislocation generates a strain field that distorts the crystal locally. The overall idea is that if the set of planes that diffracts the beam also happens to be locally distorted due to the presence of the dislocations, then contrast will arise. Given that the lattice distortion is the most pronounced near the core of the dislocation, this means that one can observe by TEM the dislocation line inside the sample (see Figure 27). Additionally, the lattice distortion will be affected by the dislocation's Burgers vector. Hence by investigating which diffraction conditions give rise to contrast, one can identify the Burgers vector of individual dislocations. Experimentally, dislocation observation is achieved by performing one of the diffraction contrast techniques described in Section 10.2.3.2 (bright field, dark field or weak beam dark field), and selecting a $\mathbf{g}$ vector that satisfies $\boldsymbol{g} \cdot \boldsymbol{R} \notin \mathbb{Z}$. When $\boldsymbol{g} \cdot \boldsymbol{R} \in \mathbb{Z}$ the dislocation will be out-of-contrast, this is why this is often referred to as the 'invisibility criterion'. $\boldsymbol{R}$ denotes the displacement field of the dislocation, which represents the distortion of the lattice, and takes the form (for a dislocation in an isotropic medium):

$$
\boldsymbol{R}(r, \phi)=\frac{1}{2 \pi}\left(\boldsymbol{b} \phi+\frac{1}{4(1-v)}\left(\boldsymbol{b}_{\boldsymbol{e}}+\boldsymbol{b} \times \boldsymbol{u}(2(1-2 v) \ln r+\cos 2 \phi)\right)\right)
$$


where $\boldsymbol{b}$ is the Burgers vector of the dislocation, $\boldsymbol{b}_{\boldsymbol{e}}$ is the edge component of the Burgers vector, $\boldsymbol{u}$ is the line direction of the dislocation, $v$ is the Poisson's ratio, and $r$ and $\phi$ are the polar coordinates. We can see that for dislocations, the expression is rather complicated as $\boldsymbol{g} . \boldsymbol{R}$ will depend on $\boldsymbol{g} . \boldsymbol{b}$ and $\boldsymbol{g} . \boldsymbol{b} \times$ $\boldsymbol{u}$. A simplification of the invisibility criterion is that the defect will be out-of-contrast when $\boldsymbol{g} \cdot \boldsymbol{b}=0$, bearing in mind that some residual contrast may arise from the term $\boldsymbol{g} . \boldsymbol{b} \times \boldsymbol{u}$. In Figure 27 this criterion was used to differentiate edge $\left(\boldsymbol{b}=\boldsymbol{a}=\frac{1}{3}[11 \overline{2} 0]\right)$ to $\operatorname{mixed}\left(\boldsymbol{b}=\boldsymbol{a}+\boldsymbol{c}=\frac{1}{3}[11 \overline{2} 3]\right)$ dislocations: edge dislocations will be visible when $\boldsymbol{g}=11 \overline{2} 0$ (left image) but not when $\boldsymbol{g}=0002$ (right image), while mixed dislocations will be visible under both conditions.

\section{Core identification by HR-STEM}

Alternatively, HR-STEM in plan-view geometry can be employed to identify the Burgers vector of dislocations. Dislocations are therefore seen end-on and the resolution of the technique allows one to observe the atomic arrangement near the dislocation. The Burgers vector of the dislocation can therefore be obtained by drawing a Burgers circuit around the dislocation (see Figure 28). Bear in mind that you will obtain the projection of the Burgers vector in the viewing plane, so that any component of the Burgers vector parallel to the zone axis will not contribute to the Burgers circuit. Hence, unfortunately, Burgers circuit drawn around edge and mixed dislocations will appear similar, and no component of the Burgers vector of a screw dislocation will be visible.

However, the real asset of HR-STEM is that one can identify the core configuration of the dislocation, as depicted in Figure 28. The core configuration of the dislocation depends in part on the dislocation type, but also can reveal other information about the material, such as the amount of strain, atomic segregation, etc. However, to be able to draw meaningful conclusions this approach requires identification of a statistically significant number of defects per sample. Even so, the problem of projection persists and the distinction between edge and mixed dislocations can be achieved by observing the Eshelby twist [108]. The Eshelby twist is an apparent rotation of the lattice that is caused by the relaxation of stresses at the free surface. This is due to the screw component of a dislocation. Hence mixed and screw dislocations will exhibit an Eshelby twist, while edge dislocations will not, which can be used to discriminate them in HR-STEM images.

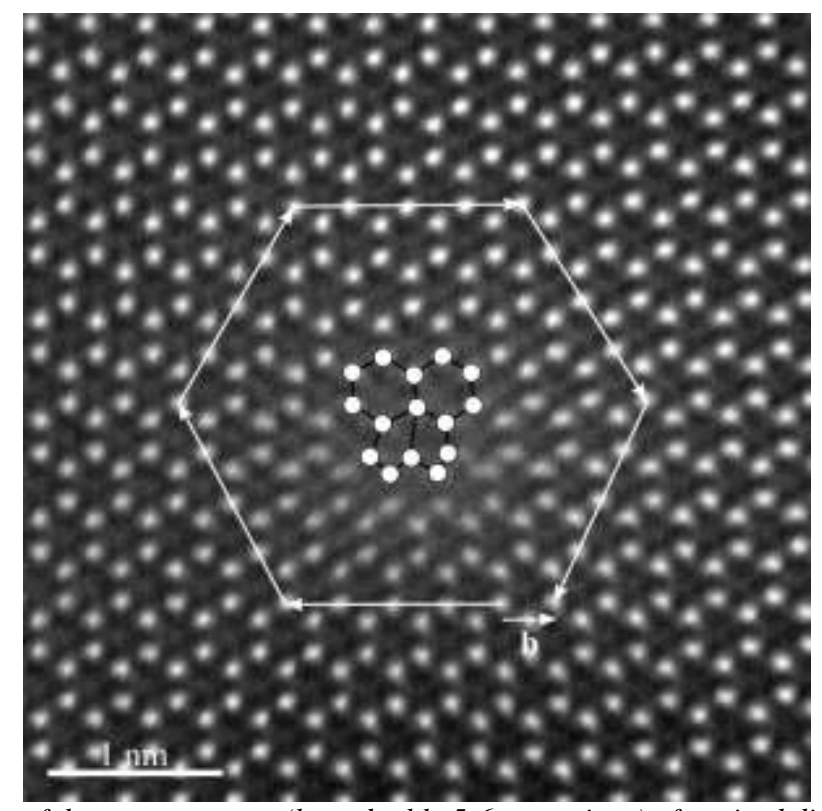

Figure 28: HR-STEM image of the core structure (here double 5-6 atom rings) of a mixed dislocation in AlGaN observed along [0001]. The white arrows indicate a Burgers circuit around the core.

\subsubsection{Intercomparison of the various methods}

It is possible to achieve accurate quantification of dislocation densities and Burger's vectors using SPM, SEM, or TEM. Indeed, careful comparisons have been made of the dislocation densities recorded using AFM on $\mathrm{SiH}_{4}$-treated surfaces and plan view TEM on the same sample, and the results are consistent between the different technqiues ${ }^{92}$. Similarly, comparisons have been made between the results of ECCI and $\mathrm{AFM}$ on $\mathrm{SiH}_{4}$-treated surfaces, not just on the same sample, but on the same defects in the same sample, and again good agreement has been achieved ${ }^{65}$. However, the different techniques have different 
strengths and weaknesses, and not all samples will yield accurate results using all techniques. One factor which limits each technique is the fact that separate dislocation-related features tend to overlap as the dislocation separation is reduced. In ECCI, it is possible to discriminate between TDs if they are more than ca. $80 \mathrm{~nm}$ apart. For AFM using the $\mathrm{SiH}_{4}$ treatment, this spacing is somewhat lower, with mixed dislocations separated by about $50 \mathrm{~nm}$ and edge dislocations separated by about $40 \mathrm{~nm}$ being distinguishable ${ }^{92}$. For AFM on untreated surfaces, using a very sharp tip, it is possible to reduce these value further, but at some cost in terms of the reliability of the measurement, since tip blunting can quickly make it impossible to resolve the tiny edge dislocation pits.

Pragmatically, AFM on treated surfaces and ECCI remain effective as a mean of both measuring dislocation density and assessing Burger's vectors up to a dislocation density of mid $10^{9} \mathrm{~cm}^{-2}$. In high resolution plan view STEM, where the individual dislocation cores can be imaged, the minimum spacing at which separate dislocations can be resolved is clearly far smaller. It should be noted however, that in cross-sectional TEM, even when the WBDF technique is used to minimize the width of the dislocationrelated features, projection effects may cause contrast from dislocations separated from one another in the through-sample-thickness direction to overlap, limiting the effectiveness of this approach in quantification at very high dislocation densities.

Although high resolution STEM provides the ultimate resolution for samples with very high dislocation densities, increasingly the GaN used in technological applications has a much lower concentration of defects. When assessing materials grown by hydride vapour phase epitaxy [109] (with dislocation densities less than $10^{8} \mathrm{~cm}^{-2}$ ) or ammonothermal growth [93] (with dislocation less than $10^{6} \mathrm{~cm}^{-2}$ ), the small area typically sampled by plan-view TEM can mean that not one single dislocation is found in the field of view. In these low dislocation density samples, SEM-based methods such as ECCI or CL present a huge advantage in being able to assess a large area quickly and easily. AFM on treated samples can address large $\operatorname{areas}^{112}$ (e.g. $30 \mu \mathrm{m} \times 30 \mu \mathrm{m}$ ), although this approach is very slow. It is also very reliant on the $\mathrm{SiH}_{4}$ treatment. This can be applied as a post-growth treatment to materials grown by other techniques, but that reduces the convenience of this approach. Overall, the availability of a range of techniques to suit the requirements of different experiments is rather useful, with either ECCI or AFM being particularly useful for fast-turnaround estimation of dislocation density, with minimal sample preparation, and STEM being well-suited to more in-depth studies of the mechanisms by which dislocations interact.

\subsubsection{Imaging of the impact of dislocations on materials properties 10.3.2.1 The interaction of dislocations with doping}

In considering the interaction of dislocations with doping in gallium nitride two essentially separate issues arise: (1) Methods for the reduction of the dislocation density lead to local changes in intentional or unintentional dopant incorporation, so that variations in dislocation density are often also related to variations in doping density. (2) Dopants, whether intentional or unintentional may segregate to dislocation cores, or to strained regions adjacent to dislocation cores. The two issues overlap to some extent where the dopant segregation affects the movement or bending of dislocations, hence influencing dislocation density reduction mechanisms. However, we shall address separately how microscopy can contribute to understanding of each of these issues, and in so doing provide short cases studies on (for the former) the application of SCM in understanding impurity incorporation and (for the latter) the application of TEM techniques to understand the influence of impurity species on dislocation cores.

Many methods for the reduction of dislocation densities in the heteroepitaxial growth of GaN involve the formation of 3D islands which are then coalesced to produce a 2D film. Dislocations tend to bend towards the inclined surfaces of the islands in order to shorten their line-length in the crystal and hence reduce the total energy. When the islands coalesce, bent dislocations may then meet and either annihilate, or react producing a new threading dislocation. Either process may reduce the dislocation density. The formation of 3D islands may be induced by altering the growth conditions during the initial stages of growth on the foreign substrate [94]. Alternatively, a GaN film with a high dislocation density may first be grown and then a mask of some sort used to prevent further 2D growth and induce 3D growth on the unmasked areas. Such a mask may be self-assembled - for example incomplete layers of $\mathrm{SiN}_{\mathrm{x}}$ deposited in situ during MOVPE growth are commonly used - or may be deposited and deliberately patterned ex situ to provide a regular array of masked and unmasked regions [110]. The most common variant on the latter process is the "epitaxial lateral overgrowth" or ELOG method in which a GaN layer is patterned with stripes of an amorphous material, usually $\mathrm{SiN}_{\mathrm{x}}$ or $\mathrm{SiO}_{2}$ which acts as a mask [111]. In one popular variant, facetted ELOG (FACELOG), the V:III ratio and temperature are reduced to 
encourage the growth of facetted stripes in the unmasked regions, forming triangular prisms of GaN usually aligned along [1-100]. Dislocations tend to bend towards the inclined side facets to shorten their line length in the crystal. Once the growth of the triangular prism structures is complete, the V:III ratio and temperature are increased, and a flux of a Magnesium precursor may be added to the growth environment. All these changes tend to encourage lateral growth, overgrowing the mask stripes, so that the prisms of $\mathrm{GaN}$ coalesce to form a flat film. At the coalescence boundaries, dislocations meet and may annihilate.

Figure 29(a) shows a schematic of this growth process, and may be compared to the TEM image in Figure 29(b) which reveals the impact of the facetted growth and lateral overgrowth on the dislocation structure [112]. Figure 29(c) and 29(d) show SCM images of the same sample, providing the phase and amplitude of the SCM signal respectively [113]. The SCM phase data shows that the GaN triangular prisms formed in the initial growth stage are n-type (appearing black), whilst the regions where lateral coalescence has occurred are p-type (appearing white). The latter point might seem unsurprising since a $\mathrm{Mg}$ prescursor was employed to enhanced the lateral growth rate. However, the p-type material has not been deliberately annealed, and so the acceptors are expected to be compensated by hydrogen. However, a clear signature of p-type conductivity is seen. The SCM amplitude data, reveal that there are variations in the concentration of the electrons in the n-type region, with detailed analysis [113] suggesting that during growth there was a higher concentration of n-type dopant incorporation on semipolar inclined $\{11-22\}$ facets than on the (0001) facet. The donor in this case is unknown: SCM does not give information about the chemical nature of the dopant. It may be $\mathrm{Si}$ (from the mask) or oxygen (from the growth environment).
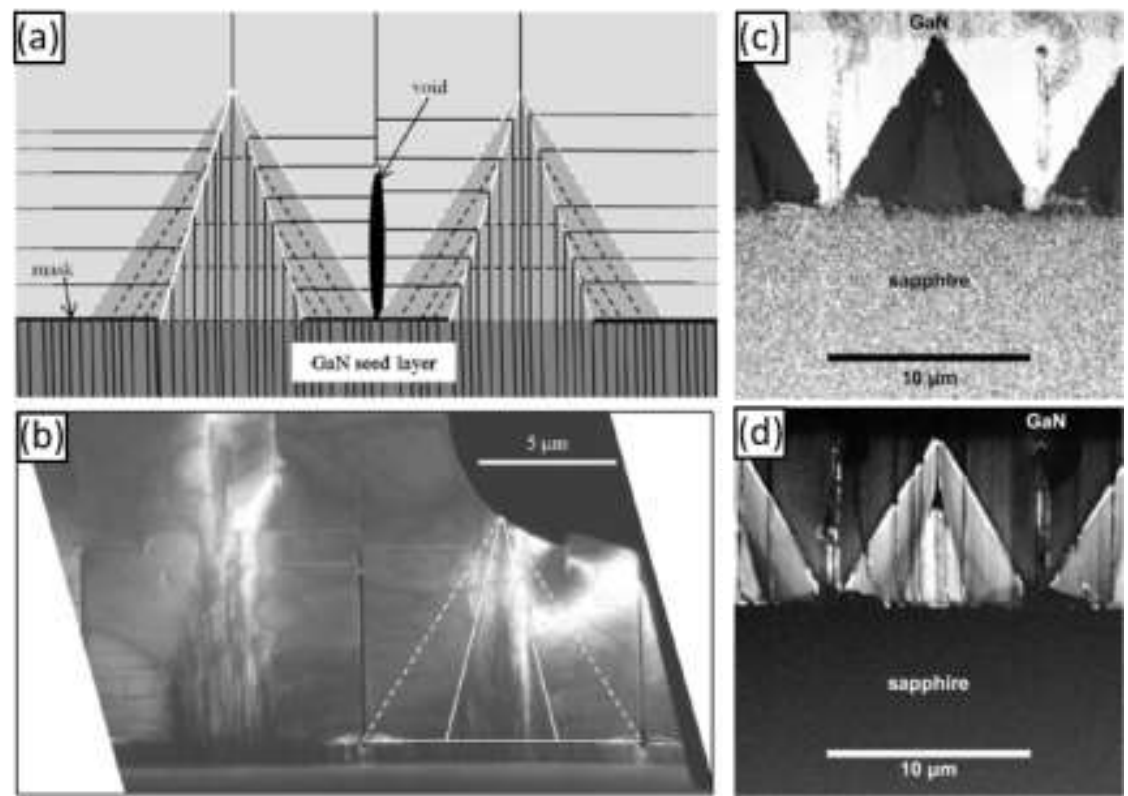

Figure 29: Dislocations and unintentional doping in GaN grown by a FACELOG technique: (a) Schematic, in which dislocations are shown as thin dark lines. (b) (0002) WBDF TEM image in which the white lines on the right-hand half of the image correspond to the white lines in (a). (c) SCM phase image in which black regions indicate n-type doping and white regions indicate p-type doping. (d) SCM amplitude image which provides information on the carrier concentration variation across the structure. (All images are cross sections). (a) and (b) reprinted from [112], with the permission of AIP publishing. (c) and (d) reprinted from [113], with the permission of AIP publishing.

The observation that $\mathrm{n}$-dopants are incorporated unintentionally on semi-polar inclined facets is ubiquitous in SCM studies of GaN samples in which dislocation density reduction is achieved using a transition from 3D to 2D growth [110]. Whist unintentional doping in GaN was, in the early days of heteroepitaxial growth studies, often attributed to point defects such as nitrogen vacancies [114], later worked showed that it is largely attributable to oxygen contamination, and SCM studies have shown that the majority of this contamination is confined to specific spatial regions in which semi-polar facets are present during growth, with the uptake of oxygen being far lower on both polar and non-polar facets. Overall, these studies illustrate that SCM is useful not only in identifying and quantifying unintentional (and indeed intentional) doping but in deriving its origins and its relationship to both point and extended defects. 
AFM-based techniques including SCM [115], conductive AFM [116] and surface photovoltage microscopy [116] has also been used to address the electrical properties of dislocation cores in materials doped with different species. However, to understand the impact of doping on dislocation core structure, TEM techniques are far more appropriate. As shown in Section 10.2.3.3, high resolution STEMHAADF imaging may be used to resolve the core structure of dislocations. The structure of dislocation cores in undoped GaN have been identified by this approach: a-type dislocations typically form a 5/7atom ring structure as illustrated in Figure 30(a-c) [117]. This experimental finding is consistent with theoretical predictions of the stable dislocation structure made using both density functional theory [118] and multiscale modelling [119] approaches. Neither n- nor p-doping leads to changes in the observed structure of a-type dislocations $[117,120]$. For a+c dislocations, on the other hand, both dissociated and undissociated dislocation cores are seen, with approximately equal frequency in undoped material. The undissociated cores exhibit a double 5/6-atom ring core structures, illustrated in Figure 30(d-f). A variety of dissociated core structures have been observed experimentally, one example of which (a 7/4/8/4/9 atom ring) is shown in Figure 30(g-i). Cores of different dissociated length may be observed, with the number of 4/8-atom rings increasing as the core length increases. Whilst Si-doping has little effect on these core structures [120], the addition of Mg-doping led to a much reduced occurrence of dissociated cores which has been attributed to the segregation of $\mathrm{Mg}$ to the tensile region adjacent to the core, which reduces the dislocation line energy and hence the driving force for dissociation [117]. These studies, the conclusions of which are supported by consistent findings from molecular dynamics simulations, illustrate the power of HAADF-STEM in identifying not only the
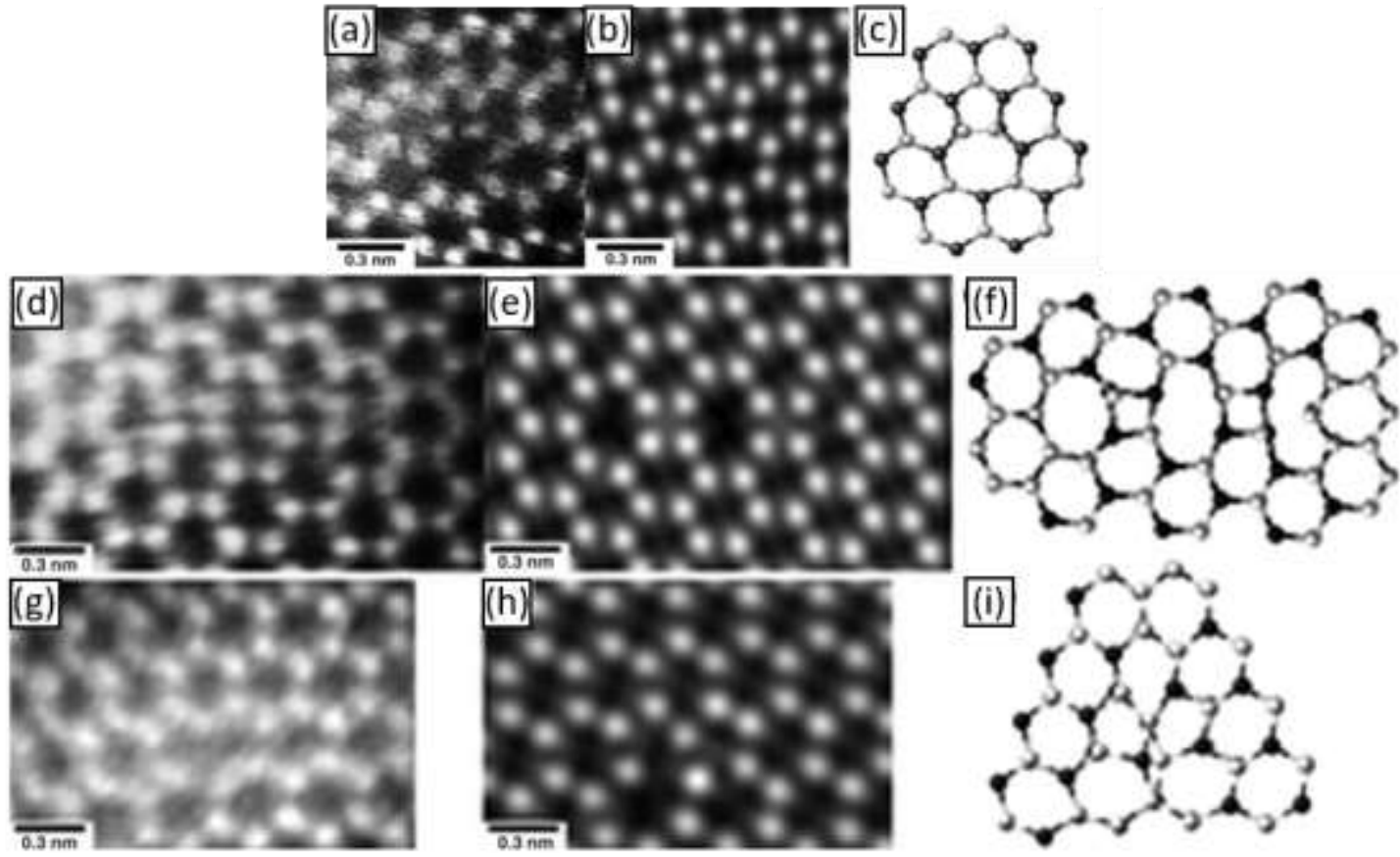

Figure 30: Typical STEM-HAADF images of (a) an a-type dislocation core, (d) an (a+c)-type dissociated core with a 7/4/8/4/9 atom ring structure and $(g)$ a 5/6-atom $(a+c)$-type undissociated core. $(b)$, $(e)$ and $(h)$ are corresponding simulated STEM-HAADF images for $(a),(d)$ and $(g)$ respectively. Similarly $(c)$, $(f)$ and $(i)$ show the relevant core structures for each row obtained from molecular dynamics simulations, in which the white spheres represent Ga atoms and the dark spheres represent $N$ atoms. Reprinted figures with permission from [117]. Copyright (2013) by the American Physical Society.

core structure, but also variations in the local chemical composition (in this case by inference rather than direct observation) which can profoundly affect the impact of the dislocations on the local electrical properties.

\subsubsection{Combining ECCI and CL to investigate the influence of defects on light emission}

Although, as was explained in Section 10.3.1.1, GaN-based optoelectronic devices are remarkably robust to the presence of threading dislocations, it was shown, as early as 1997 , by a comparison of atomic force microscope (AFM) and cathodoluminescence (CL) images that dislocations in GaN can indeed act as non-radiative recombination centres [121]. TDs can have various effects on a material's optical and electrical properties leading to other recombination channels besides near band edge 
recombination or act as scattering centres affecting the carrier transport. The influence of the dislocation type on the optical properties, including whether they are radiative or non-radiative centres for recombination, shows strong ambiguity in the literature. Sugahara et al. have found that all types of dislocations in homo- and heteroepitaxial $\mathrm{GaN}$ act as non-radiative recombination centres for minority carriers as seen in transmission electron and CL images [122]. Hino et al. on the other hand discovered evidence in AFM, SEM and PL measurements that only dislocations with a screw-component (screwand mixed-type) act as centres for non-radiative recombination, whereas edge-type do not behave that way [123]. The opposite was reported by Albrecht et al., who found evidence in thick homoepitaxial GaN layers that only dislocations with an edge-component (edge- and mixed-type) are recombination active; meaning that only edge- and mixed-type dislocations are centres for non-radiative recombination and screw-type dislocations have no impact on the luminescence, i.e. produce no dark spots in CL image[124]. More recently Medvedev et al. showed that screw-type dislocations introduced through scratching or indentation exhibit bright luminescence, which is shifted by $0.3 \mathrm{eV}$ from the regular GaN band edge emission peak even at room temperature [125].

Besides influencing the optical properties of GaN-based devices, dislocations can also impact on their electrical performance. High current densities and low effective Schottky barrier heights were observed in areas close to dislocations having a screw-component in an n-doped GaN film [126]. Dangling bonds near edge-type dislocations in $\mathrm{AlGaN}$ form acceptor-like states, which reduce the free carrier concentration and mobility with increasing edge dislocation density [127]. The same authors also correlate a decrease of PL intensity with increasing edge-type dislocation density to these dislocations being centres for non-radiative recombination. Furthermore, a reduction of TD density showed a drastic decrease of reverse-bias leakage current in GaN p-n junctions [128].

To further investigate the optical behaviour of dislocations CL, electron channelling contrast imaging (ECCI) and AFM imaging was performed on the same area micron scale of a Si-doped GaN epilayers as displayed in Figures 31(a)-(c), respectively [65]. 

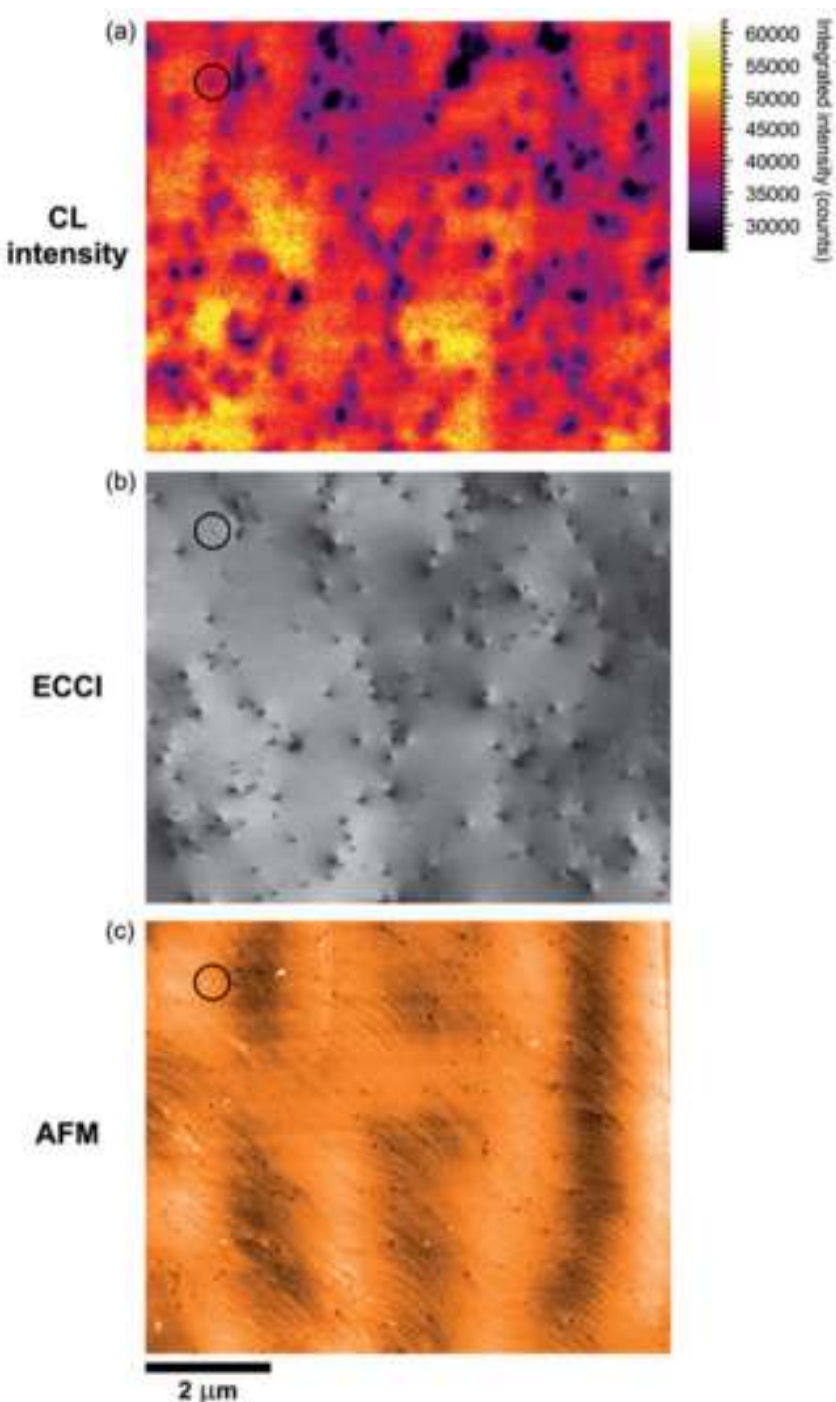

Figure 31: (a) CL intensity of the GaN band edge emission, (b) ECCI and (c) AFM images from the same micron scale area of a Si-doped GaN epilayers. Reprinted from [65] with permission from Cambridge University Press.

The CL intensity image of the GaN near band edge emission shows a large number of dark spots, associated with non-radiative recombination at TDs [121,122]. They have varying diameter and appear in clusters of two or more dislocations in close proximity, which can lead to an underestimation of the TD density. The $\mathrm{CL}$ measurement was performed at a relatively high current $(5 \mathrm{kV}, \approx 6 \mathrm{nA})$ in order to excite an intense enough $\mathrm{GaN}$ band edge emission due to carbon contamination from the previouslyperformed ECCI measurement on the same area leading to larger observed black spots.

From several ECCI images it was determined that $60 \%$ of the dislocations are edge-type, fewer than $2 \%$ are screw-type and the remainder are mixed-type with a total TD density of $(5.1 \pm 0.4) \times 10^{8} \mathrm{~cm}^{-2}$. It is possible to discriminate between TDs in the ECC image in Figure 31(b) if they are further than approximately $80 \mathrm{~nm}$ apart. For the investigated area, approximately 750 TDs were found using the ECCI technique, with about 400 TDs being resolved as single dislocations in the CL image. Careful comparison of the images in Figure 31(a) and (b) shows a one-to-one correlation of the dark spots in the CL image and spots with B-W contrast in the electron channelling image for those single dislocations.

AFM imaging (Figure 31(c)) of the same area was performed to confirm that all dislocations were visible in the ECC image for the selected channelling conditions. The AFM studies utilised the $\mathrm{SiH}_{4}$ treatment described in Section 10.3.1.2. Careful comparison identified isolated dark spots corresponding to single dislocations in the CL image to be TDs of both edge- and mixed-type in the AFM image. Therefore, it can be concluded that both pure edge dislocations and TDs with a screwcomponent act as centres for non-radiative recombination in the investigated $\mathrm{Si}$-doped $\mathrm{GaN}$ layer. The results are inconclusive about pure screw dislocations acting as non-radiative recombination centres, 
because their density ( $<2 \%$ of the total dislocation population) is not high enough to identify single and isolated screw TDs for a comparative measurement.

Other examples of combining luminescence information from CL with structural information from ECCI are the investigation of growth temperate of GaInP on TDs [70], grain boundaries in CdTe thin film solar cells [129] or the investigation of recombination behaviour of dislocations in III-V photovoltaic materials using electron beam-induced (EBIC) instead of CL [130].

\subsubsection{A multi-microscopy example: structure, properties and interactions of dislocations in InGaN}

In the previous subsections we have seen how several types of information can be acquired within the same microscope (topography and SCM in the AFM, ECCI and CL in the SEM). In this section, we expand the idea of correlative microscopy by describing how various properties of the materials can be correlated using multiple separate microscopes. We refer to this approach as 'multi-microscopy', and the use of AFM alongside ECCI and CL in Section 10.3.2.2 provides a first illustration of its usefulness. We will further illustrate this approach by linking the properties of dislocations in InGaN using AFM, SEM-CL, and TEM, with a focus here on the practical aspects of how the correlative experiment can be achieved.

\section{Practical aspects}

Probably the most important aspect of the multi-microscopy approach is that identification of the same region is done with the help of one or several 'markers'. It is particularly helpful to have markers at different length scales which can significantly reduce the time required to find the same feature (here, a specific dislocation) in all the different microscopes. Here we employed, as a micro-scale marker, a TEM grid but one could also use an intentional scratch or even unintentional debris on the surface. Our nano-scale markers here are a natural feature of our sample: trench defects. The sample preparation can obviously be adjusted depending on the requirements of the experiment (especially based on which microscopes are to be used). Figure 32 shows an example for the study of threading dislocations in InGaN using AFM, SEM-CL and TEM (in that specific order!).
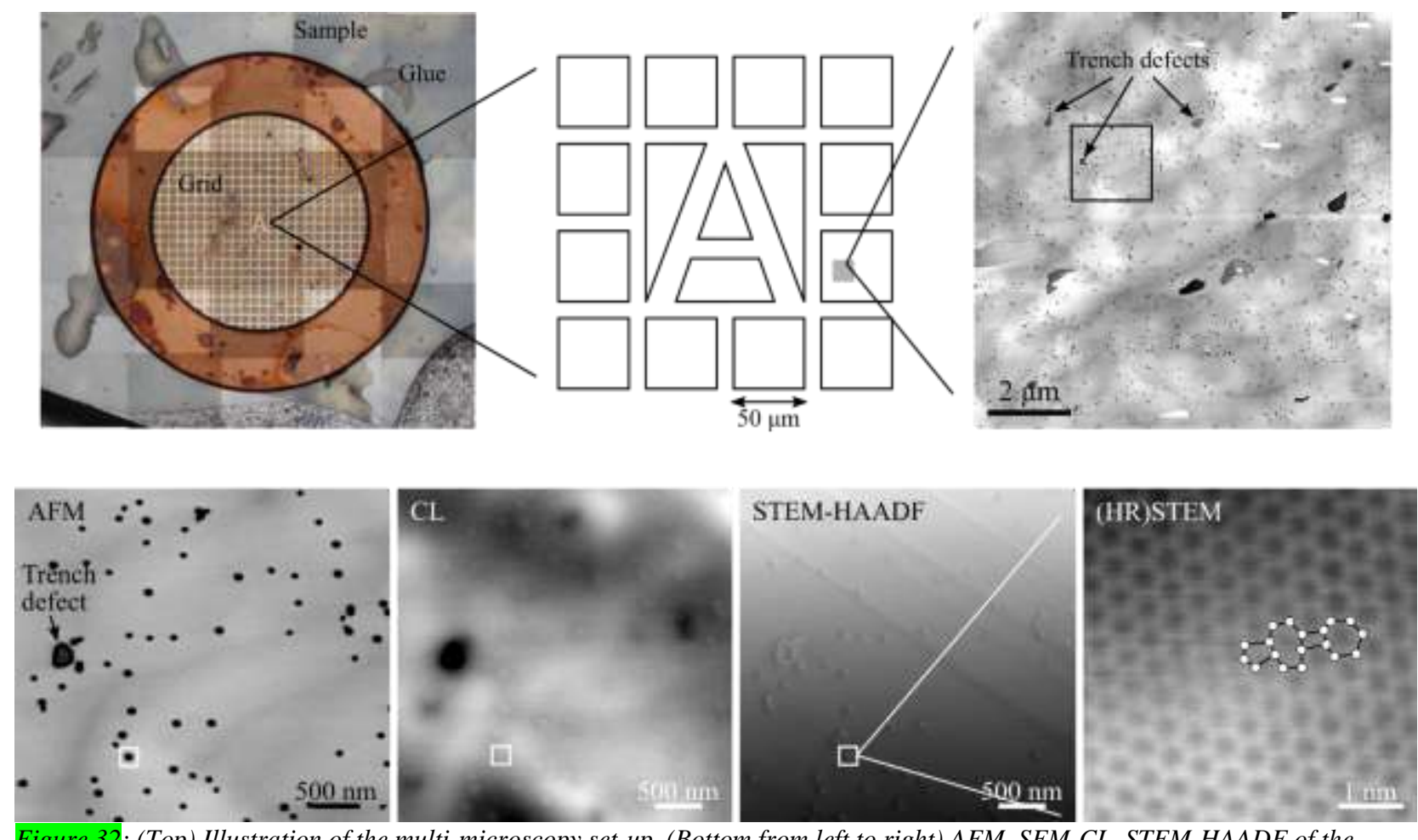

Figure 32: (Top) Illustration of the multi-microscopy set-up. (Bottom from left to right) AFM, SEM-CL, STEM-HAADF of the same micrometer-scale region of material, and HR-STEM image the dislocation indicated by a white square.

The sample is first cut to a $3 \mathrm{~mm}$ square - in anticipation of a future TEM sample preparation. Cleaning of the sample surface is highly recommended beforehand to prevent having particles between the sample surface and the grid which will be stuck to it. We used a 300 square mesh copper grid (mesh size ca. 50 $\mu \mathrm{m})$ as is usually employed for TEM sample preparation. In order to avoid damaging and bending the 
grid during manipulation with tweezers, a $2 \mathrm{~mm}$ hole copper ring is stuck onto the grid prior to any manipulation of the grid. The set-up is then stuck to the surface of the sample by putting four small drops of glue at the edges of the set-up, and positioned so that the grid (not the copper ring) is in contact with the sample surface. It is important to put only small drops of glue outside the grid, and not below it, in order to avoid having the glue spreading under the grid, which would increase the grid-sample distance and eventually also cover the regions of interest. It is crucial that the grid-sample distance remains as low as possible otherwise the AFM tip may not be able to reach the sample through the mesh. Once the grid is securely in place, AFM measurements may be performed.

It is recommended to perform AFM observation of the sample before SEM-CL to avoid sample contamination due to electron beam exposure. Given that the measurements performed on the AFM are often tip-dependent, you should use the same fresh tip to record all the images during a single AFM session. In the context of the study of dislocations in InGaN, AFM was used to record morphological properties, which are, for each dislocation, the size of the pit terminating the dislocation - which is indicative of the dislocation type [92] - and the distance to the nearest neighbouring dislocation. This is obviously non-exhaustive and any information that can be recorded on the AFM (e.g. capacitance, conductivity) can also be acquired. For accurate measurements on small defect-related features, typical image sizes are small $(1-3 \mu \mathrm{m})$. Once the requisite small scale images have been recorded within a particular grid square, a larger scan $(10-20 \mu \mathrm{m})$ should be taken. That will facilitate identification of the same region in the next microscope(s), in this case the SEM-CL.

In the SEM, the first requirement is to find the same region which was analysed in the AFM, using the micron-scale markers and overview scan. In this example, SEM-CL was used to record the optical properties - intensity and emission wavelength - of the dislocations previously analysed by AFM and compared to that of the surrounding defect-free material. Again, an overview SEM image of the whole $50 \mu \mathrm{m}$ square should be recorded at the end of the experiment to help with identification of the approximate position of the region of interest within the square during TEM sample preparation.

Our approach to preparation of the sample for plan-view TEM in this multi-microscopy example follows the standard mechanical polishing preparation procedure (as outlined in Box 1 in section 10.2.3.1), although there are a couple of points to note. First, the grid-sample holder has to be reinforced with more glue, otherwise it won't sustain the mechanical stress applied during mechanical grinding. Glue can be placed anywhere on the grid, as long as the regions of interest are avoided. The second point, perhaps the most critical, has to do with the ion milling. Here it is crucial to position the centre of rotation of the ion mill as close as possible to the region of interest. The idea is to minimise the time spent milling once the hole has formed - otherwise it will blunt the edges of the hole, and sputtering of the grid to the sample may occur. One should be extremely careful and stop the ion milling when the hole forms. After formation of the hole, it is recommended to use a low voltage (e.g. 1-3 kV) ion beam in single modulation mode in order to enlarge the hole towards the region of interest. Initial TEM imaging is needed to check whether the region of interest is electron-transparent. If not, the gentle ion milling step must be repeated. It is highly unlikely that the whole region of interest will be suitable for TEM analysis at the same time (the foil thickness will vary across the region of interest). Hence, several iterations of gentle ion milling and TEM observation will be required in order to be able to analyse as many defects as possible. In this example, the atomic configuration of the dislocation core was obtained by TEM - as illustrated in Figure 32.

\section{Results}

The extensive dataset thus generated can now be analysed to extract potential correlations between the morphological, optical and structural properties of each dislocation. Figure 33 illustrates the multimicroscopy approach, whereby we can obtain general trends over the entire dataset involving data from several microscopes. Here we correlate the intensity ratio (which is the CL emission intensity at the dislocation compared to that of the surrounding defect-free material) which we obtained by CL, to the distance to nearest neighbour which we measured by AFM. The dataset is also separated into edge and mixed dislocations, which were identified by analysis of the AFM data. Interpretation of such trends (along with other trends not shown here) enabled us to highlight the segregation of In atoms near the core of dislocations, which leads to the bright spot seen in the CL image in Figure 32, in contrast to the dark spots seen where dislocations intersect MQWs and form V-pits in Section 10.2.2.4. More details about the analysis can be found in reference [131]. 
The strength of the multi-microscopy approach is also the ability to extract individual data points from the trend and analyse them further. For example, in Figure 33 two mixed dislocations with similar properties (low intensity ratio and small distance to nearest neighbour) have been selected and analysed by TEM. Despite their similarities in AFM and SEM-CL, their core configurations are intrinsically different - one is dissociated (left image) and the other one is undissociated (right image). This highlights that the core structure of a dislocation (in $\mathrm{InGaN}$ ) does not dominate its optical properties.

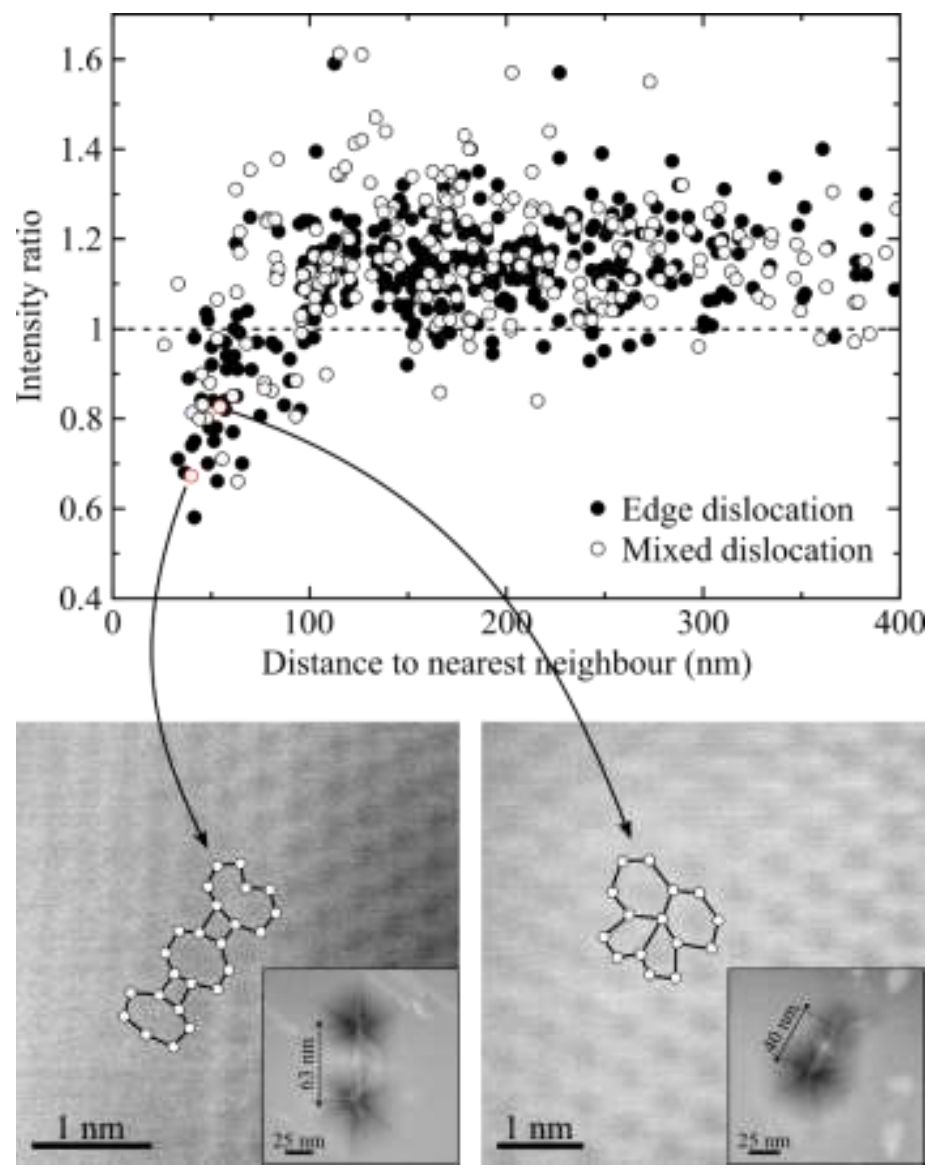

Figure 33: (Top) Plot of the intensity ratio (measured by CL) versus distance to nearest neighbour (measured by AFM) as a function of the dislocation type (obtained by AFM). (Bottom) HR-STEM images of the core of the dislocations corresponding to the data points highlighted in the top graph. In inset, zoom-out HAADF-STEM image of the dislocation.

\subsubsection{Imaging defects in 2D materials}

\subsubsection{Addressing the electrical properties of defects in $2 D$ transition metal dichalcogenides by scanning probe microscopy}

Layered materials such as $\mathrm{NbSe}_{2}$ and $\mathrm{WSe}_{2}$ which can be mechanically exfoliated to produce twodimensional (2D) semiconductor samples have long been attractive to researchers using STM to study defects since cleavage provides a straightforward means of producing clean, atomically-flat surfaces [132]. As the popularity of 2D materials has increased, so has the breadth of SPM studies, expanding to encompass not only mechanically exfoliated samples but also materials grown by chemical vapour deposition [133]. Furthermore, a wide range of SPM techniques have been applied, including C-AFM (which can resolve atomic-scale periodicity in samples imaged under a dry nitrogen atmosphere [134]), KPFM and differential surface photovoltage microscopy [133]. STM remains, however, an extremely powerful approach to the identification of defects in 2D materials and the assessment of their impact on electronic structure. A wide variety of atomic scale features are observed in STM images, believed to be linked to point defects and/or impurities, which are influenced (as is usual) by both topographic and electronic contributions, which complicate image interpretation [132, 135]. Identification of the defect is somewhat more straightforward for dislocations, since at low angle grain boundaries (for example) the misorientation of the atomic planes in adjacent regions provides a clear signature of the nature of the defects at the boundary, independent of the atomic resolution images of the defects themselves. 
Huang et al. [136] imaged dislocations at low angle grain boundaries in $\mathrm{WSe}_{2}$, and observed an array of dislocations characterised by bright "butterfly features" - i.e. pairs of bright lobes located symmetrically on either side of the boundary (see Figure 34). These butterfly features had significant apparent height when the boundary was imaged at a negative tip bias (Figure 34(a-c)), but with a positive tip bias no such protrusion was observed (Figure 34(d)). This suggested that the apparent protrusion was actually an electronic feature, linked to redistributions of the LDOS at the defects, rather than out-of-plane lattice deformations. STS revealed the presence of states in the band gap between the Fermi-level and the conduction band minimum. These unoccupied states will be imaged at negative tip bias, when the electrons tunnel into them, and explain the bright features observed in imaging under these conditions. A number of possible dislocation core-structures were proposed and the projected density of states was simulated with density functional theory. A 4/6 atom ring core structure was identified which allowed good agreement between simulated and experimental STM images and also consistency with the STS data in terms of the gap states. Low angle grain boundaries subjected to external strain were then modelled based on these structures, and the models suggested that strain can be used to modify the gap states and hence the electrical impact of the grain boundaries on devices.

It is important to note here that the comparison of images predicted by atomistic modelling with real experimental data was key to identification of the defect structure, and there are clear parallels to the identification of dislocation core structures in GaN using STEM-HAADF data (Section 10.3.2.1), despite the very different nature of both the imaging technique and the material under study. Equally, the use of STS and voltage dependent imaging to identify features linked to unoccupied states is a similar approach to that which was applied to the understanding of point defects in GaAs in Section 10.2.1.3.

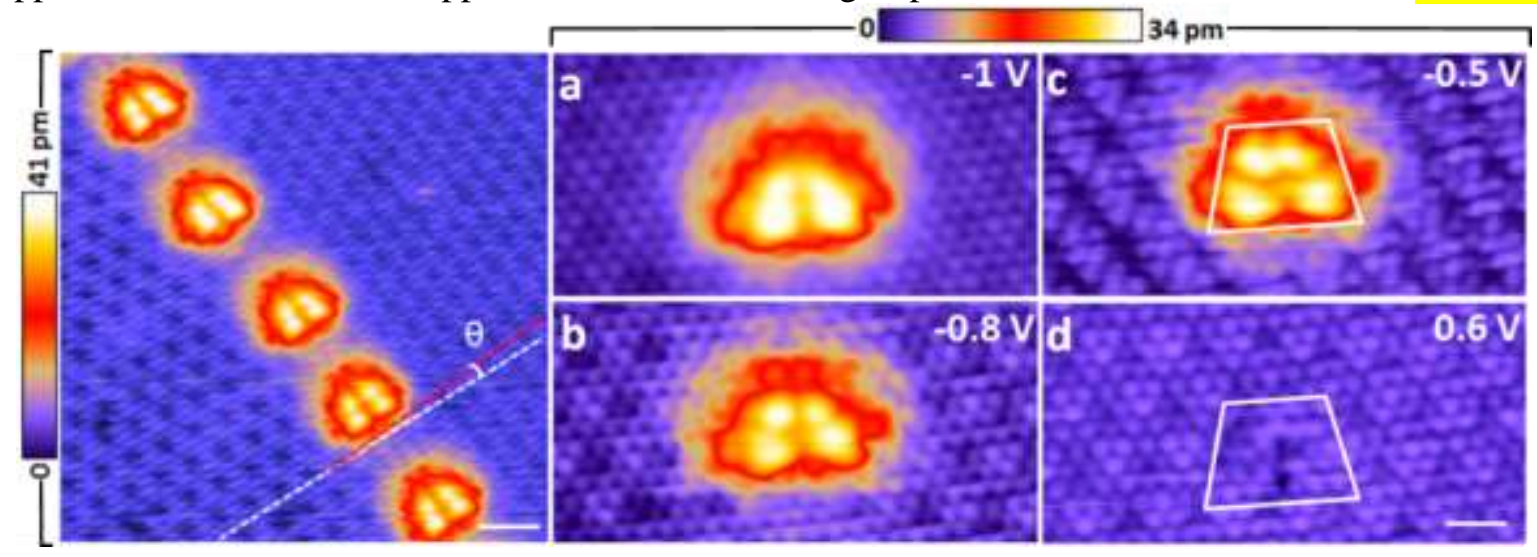

Figure 34: STM images of a low angle grain boundary in WSe 2. Leftmost image: Overview of a boundary with a misorientation angle, $\theta$, of $4.5^{\circ}$, displaying an array of butterfly features corresponding to dislocations. Scale bar: $2 \mathrm{~nm}$. (a) - (d) Images of a particularly butterfly feature recroded under different tip biases. Note that the apparent protrusion disappears when the tip is positively biased relative to the sample, accessing the occupied states of the sample. Scale bar: $1 \mathrm{~nm}$. Reprinted (adapted) with permission from [136]. Copyright (2016) American Chemical Society.

\subsubsection{ECCI examination of graphene nucleation on C-Face silicon carbide}

Investigating defects in 2-D films using ECCI is an enticing prospect. To obtain images in such thin films requires that the penetration depth of the electron beam, and therefore the energy of the incident electron beam, be set to as low as is possible when acquiring an ECCI micrograph. It has been shown that the channelling contrast is greater at lower beam energies [21]; however the brightness of the electron source decreases with beam energy and this, coupled with the decreasing performance of the electron sensitive diodes with energy [21], means that in practice the lowest energies used to acquire ECCI micrographs are of order $10 \mathrm{keV}$.

In spite of this, attempts have been made to analyse 2-D films with ECCI. One interesting result has been published by Hite et al. [137] where the initial stages of epitaxial graphene growth were studied by characterization of graphene formed in localized areas on C-face $6 \mathrm{H}-\mathrm{SiC}$ substrates. Their SEM studies revealed that the initial growth begins with small, hexagonal 'basins' recessed into the SiC substrate. These graphene-covered basins (GCBs) are produced through the sublimation of Si on the formation of the graphene. ECCI revealed that the graphene growth nucleated around threading screw dislocations in the $\mathrm{SiC}$ substrate, see Figure 35. 

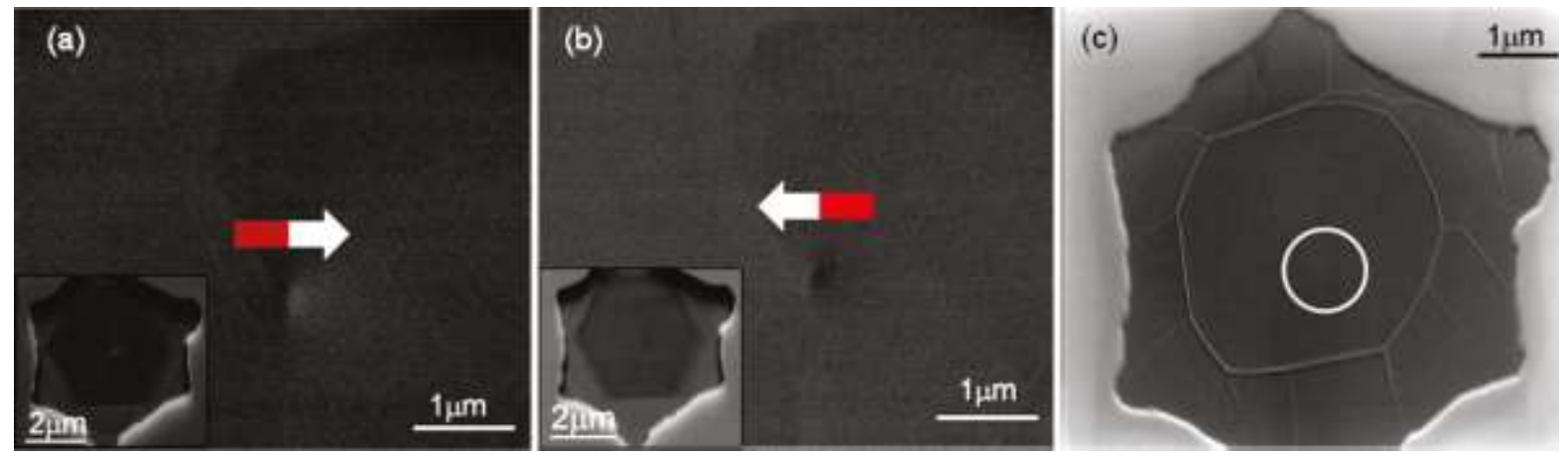

Figure 35: Magnified ECCI image of a screw dislocation in the center of a GCB, with an arrow indicating the direction of the dark-to-light contrast. The inset shows an image of the entire GCB. (b) Same GCB, only tilted to the opposite side of the Bragg angle $\left(0.4^{\circ}\right.$ difference - behavior that confirms the nature of the threading screw dislocation. The arrow indicates that the dark-to-light contrast has changed direction. (c) Secondary electron detector image of the same GCB shows giraffe stripes and no features where the TSD is located (circled), proving that the signal at the TSD location is not a morphological feature (Reprinted with permission from [137]. Copyright 2011 American Chemical Society).

Future developments in SEM and electron detector performance will lead to many more SEM studies of 2-D materials.

\subsubsection{Dislocation and point defect identification in h-BN by TEM and future outlook using machine learning}

Hexagonal boron nitride (h-BN) is a wide band gap semiconductor (ca. $6 \mathrm{eV}$ ) which exhibits single photon emission in the visible and near ultraviolet part of the spectrum $[138,139]$. This emission is currently thought to occur at defect sites but no direct correlation between a specific defect type and emission property has so far been demonstrated. This is indeed a challenging task. A first step is to demonstrate an ability to identify the structure and chemistry of such defects - using TEM.

2D materials are of course atomically thin. This indicates that all kinds of atomic scale defects stretch through the entire (single atom) thickness of the material. This property is particularly interesting for the study of point defects, as in 3D materials these are extraordinarily difficult to identify by TEM due to their small size comparative to the thickness of the TEM foil. The main difficulty for imaging atomic scale defects in 2D materials is beam damage - especially for those made of light element, i.e. graphene and h-BN. As we have seen in Section 10.2.3.1 the maximum energy transferred can be significant in such materials. Moreover, owing to the atomically thin nature of the materials, the displacement energy is much lower than for bulk materials. For example, they would be more prone to beam sputtering than to atomic displacement. Hence when one wants to observe 2D materials by TEM, one must employ low voltages $(80 \mathrm{kV}$ or even $60 \mathrm{kV})$ and extremely low electron dose (implying using TEM instead of STEM).

Just to name a few examples, aberration corrected HR-TEM has been used to observe the atomic structure of grain boundaries in chemical vapour deposited h-BN, which consist of a succession of 5 and 7 atom rings (Figure 36) [140]. In another study, single B vacancy in bilayer h-BN have been observed and shown to result in interlayer bonding (Figure 37) [141]. 

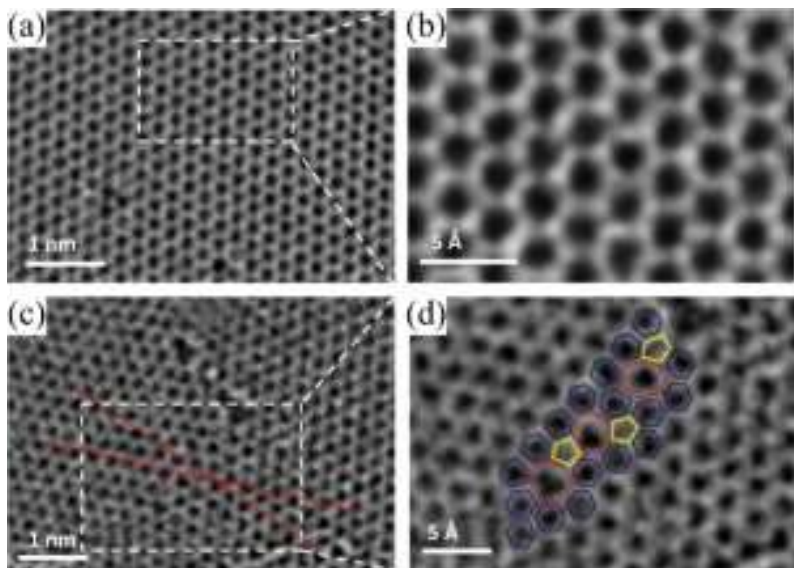

Figure 36: Aberration-corrected HR-TEM images of h-BN monolayer containing (a-b) no defect, (c-d) a grain boundary. Adapted from [140]. Copyright 2013, American Chemical Society.
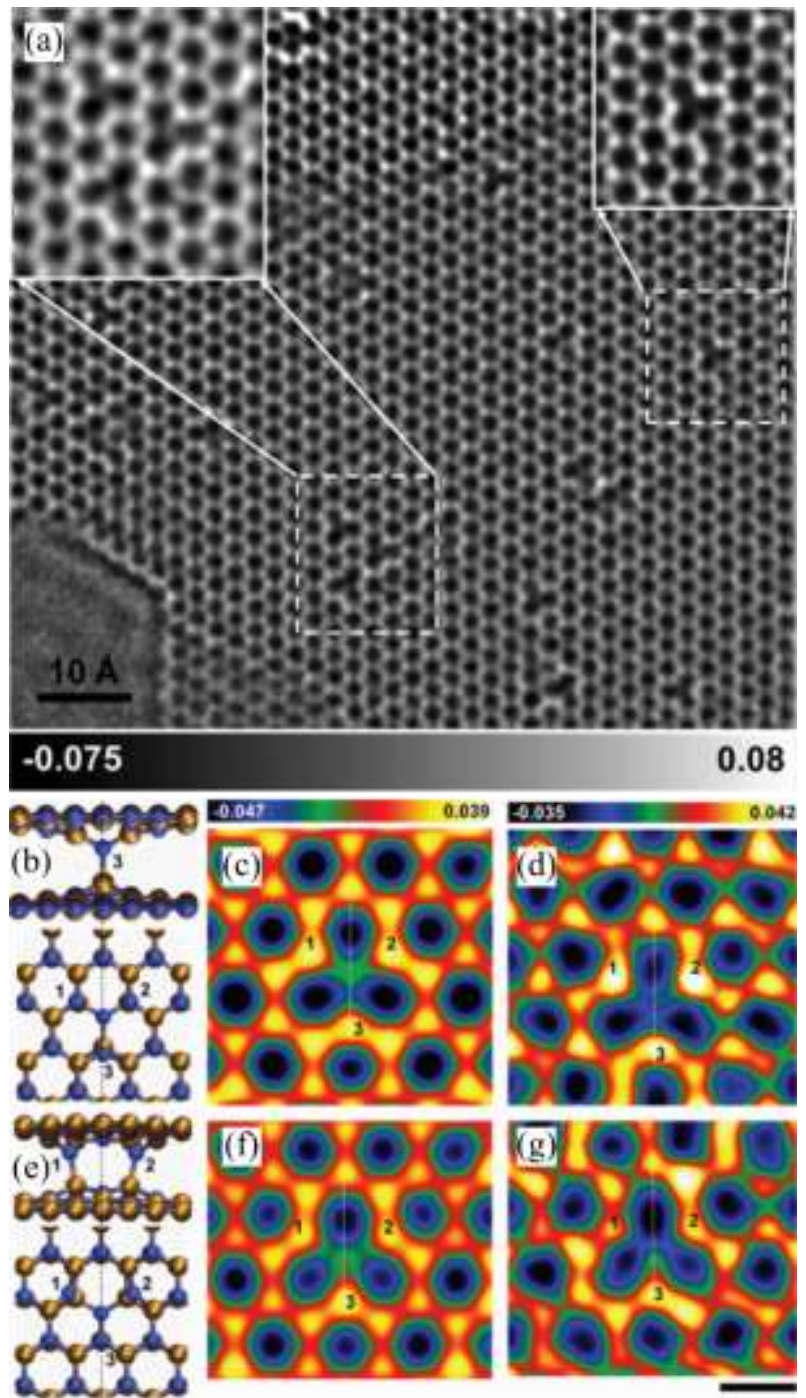

Figure 37: (a) Aberration-corrected HR-TEM images of h-BN bilayer containing B vacancies. (b) Atomic structure model, $(c)$ image simulation, and $(d)$ experimental image of vacancy with one interlayer bond. $(e-g)$ Same for vacancy with two interlayer bonds. Reprinted figures with permission from [141]. Copyright 2011 by the American Physical Society.

The increasing access to aberration-corrected microscopes will undoubtedly result in a larger volume of data of materials at the atomic scale (not necessarily limited to 2D materials). However manual identification of the atomic structure of defects is time-consuming and requires an experienced user. In 2018, we are now at the advent of artificial intelligence, machine learning and neural networks, which could make the defect identification task automated, faster, and (with proper training of the algorithm) 
unbiased and more reliable than if it were done by a human. Machine learning for image recognition is embedded everywhere in our daily life, and has now been applied to identify the structure and composition of defects in TEM [142] and STEM [143]. These algorithms can be trained from simulated images of simple defects. Even though it was trained on basic defects in graphene, a deep learning algorithm was able to identify more complex defects such as 3-fold and 4-fold coordinated Si impurities in experimental images of graphene (Figure 38). Without further training the algorithm was also able to identify defects in other materials. These studies allow us to envision a future where microscopy (not only TEM) of defects in semiconductors would be nearly fully-automated.
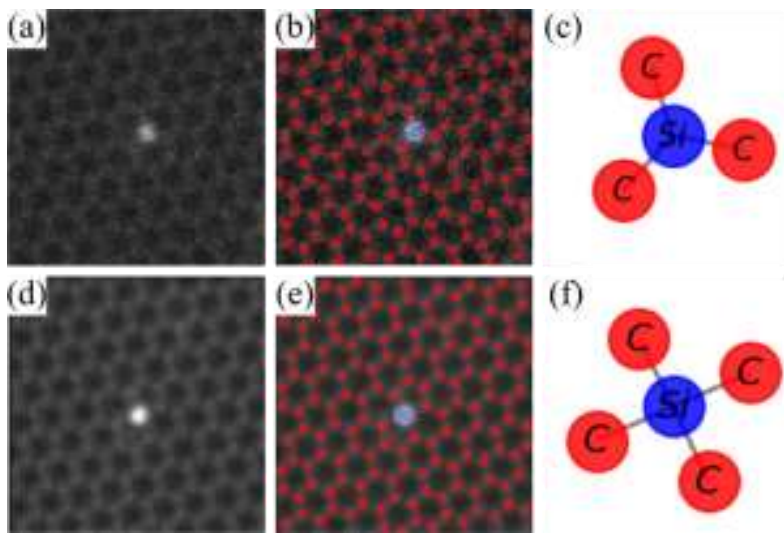

Figure 38: (a) Aberration-corrected HR-STEM image of 3-fold coordinated Si defect in graphene. (b) Output from machine learning approach for the image in (a), and (c) representation of the defect. (d-e) Same for a 4-fold coordinated Si defect in graphene. Adapted with permission from [143]. Copyright 2017, American Chemical Society.

\subsection{Conclusions and outlook}

This chapter has illustrated the enormous usefulness of microscopy techniques, particularly for the analysis of extended defects, and also, in certain circumstances for the analysis of point defects. It has also highlighted some recurring challenges, which include appropriate sample preparation, identification of artefacts, damage to the sample during imaging, achieving an appropriate resolution to address any particular problem, and achieving sufficient sampling to allow statistically significant conclusions to be made in techniques which sometimes image only very limited areas.

Despite these challenges, microscopy techniques play a vital role in the characterisation of defects in semiconductors and as they continue to develop new opportunities will arise. Although this chapter has been necessarily selective, focussing on some of the current key techniques, new methodologies are continuously being developed, and we note a number of emerging trends. In the TEM sphere, we are increasingly seeing integration of techniques for the characterisation of (opto)electronic properties of materials directly into the electron microscope. One exciting example of this is the application of CL in the TEM, where the limited interaction volumes attainable due to the use of thin film samples aids the achievement of excellent resolution [144]. Similarly, SEM-based techniques are becoming ever more sophisticated, with CL experiments in the SEM being extended to allow time-resolved as well as time-integrated experiments [145]. In SPM, the range of techniques to allow the electrical characterisation of semiconductors at the nano-scale is ever-expanding, and may increasingly be integrated with mechanical property measurements, even for very stiff materials (such as most semiconductors). Hence, nanoscale studies of semiconductors are extending to embrace piezotronic and optopiezotronic devices, where mechanical stimuli are used to manipulate the electronic and optoelectronic properties of nanostructures [146].

All of these endeavours which link materials properties to materials structure have the potential to generate vast data sets. Hence, parallel developments in machine learning and big data techniques will be vital in the future to maximise the information and understanding which can be extracted from microscopy data.

\section{Acknowledgements}

The authors would like to thank G. Naresh-Kumar, Ben Hourahine and Aimo Winkelmann for their support during the writing of this chapter. 


\section{References}

[1] Eaton P., West P. 'Atomic Force Microscopy'. Oxford University Press, 2010

[2] Binnig G., Quate C.F. 'Atomic Force Microscope'. Phys. Rev. Lett. 1986;56(9):930

[3] Timoney P., Zhang X.X., Vaid A., Hand S., Osborne J., Milligan E., Feinstein A. 'Device Level 3D

Characterization using PeakForce AFM'. Article Number: 97781A in Sanchez M.I., Ukraintsev V.A. (Eds.)

'Metrology, Inspection, And Process Control For Microlithography XXX'. Proceedings of SPIE Vol. 9778.

Bellingham: SPIE; 2016

[4] Desbief S., Hergué N., Douhéret O., Surin M., Dubois P., Geerts Y., Lazzaronia R., Leclère P., 'Nanoscale investigation of the electrical properties in semiconductor polymer-carbon nanotube hybrid materials'.

Nanoscale. 2012;4(8):2705

[5] Oliver R.A. 'Advances in AFM for the electrical characterization of semiconductors". Reports on Progress in Physics. 2008;71:076501

[6] Coffey D.C., Reid O.G., Rodovsky D.B., Bartholomew G.P., Ginger D.S. 'Mapping Local Photocurrents in Polymer/Fullerene Solar Cells with Photoconductive Atomic Force Microscopy'. Nano Letters. 2007;7(3):738

[7] Cox G., Szynka D., Poppe U., Graf K.H., Urban K., Kisielowski-Kemmerich C., Krüger J., Alexander H.

'Scanning tunneling microscopy of crystal dislocations in gallium arsenide'. Phys. Rev. Lett. 1990;64(20):2402

[8] Feenstra R.M., Woodall J.M., Pettit G.D. 'Observation of bulk defects by scanning tunneling microscopy and spectroscopy: Arsenic antisite defects in GaAs'. Phys. Rev. Lett. 1993;71(8):1176

[9] Tersoff J., Hamann D.R. 'Theory and Application for the Scanning Tunneling Microscope'. Phys. Rev. Lett. 1983;50(25): 1998

[10] Domke C., Ebert Ph., Heinrich M., Urban K. 'Microscopic identification of the compensation mechanisms in Si-doped GaAs'. Phys. Rev. B. 1996;54(15):10288

[11] Just S., Zimmermann S., Kataev V., Büchner B., Pratzer M., Morgenstern M. 'Preferential antiferromagnetic coupling of vacancies in graphene on $\mathrm{SiO}_{2}$ : Electron spin resonance and scanning tunneling spectroscopy'. Phys. Rev. B. 2014;90(12):125449

[12] Holt D.B., Joy D.C. (eds.). 'SEM microcharacterization of semiconductors'. Academic Press. 1989

[13] Goldstein J., Newbury D., Joy D., Lyman C., Echlin P., Lifshin E., Sawyer L., Michael J. 'Scanning Electron Microscopy and X-ray Microanalysis' $3^{\text {rd }}$ edn. Springer. 2007,

[14] Reimer, L. 'Scanning Electron Microscopy: Physics of Image Formation and Microanalysis'. $2^{\text {nd }}$ edn. Springer. 1998.

[15] Zhou W., Wang Z.L. (eds.). 'Scanning microscopy for nanotechnology: techniques and applications'. Springer Science \& Business Media. 2007

[16] Edwards P.R., Martin R.W. 'Cathodoluminescence nano-characterization of semiconductors'.

Semiconductor Science and Technology. 2011;26(6):064005

[17] Yakimov E.B. 'Investigation of electrical and optical properties in semiconductor structures via SEM techniques with high spatial resolution'. Journal of Surface Investigation. X-ray, Synchrotron and Neutron Techniques. 2012;6(6):887-9

[18] 'Jeol: Scanning Electron Microscope A To Z: Basic Knowledge For Using The SEM'

https://www.jeol.co.jp/en/applications/pdf/sm/sem atoz all.pdf

[19] Edwards P.R., et al. 'High-Resolution Cathodoluminescence Hyperspectral Imaging of Nitride

Nanostructures'. Microsc. Microanal. 2012;18:1212-1219

[20] Wan Q., et al. 'Angle selective backscattered electron contrast in the low-voltage scanning electron microscope: Simulation and experiment for polymers'. Ultramicroscopy. 2016;171:126-138

[21] Wilkinson A.J., Hirsch P.B. 'Electron diffraction based techniques in scanning electron microscopy of bulk materials'. Micron. 1997;28(4):279-308

[22] Trager-Cowan C., Sweeney F., Trimby P.W., Day A.P., Gholinia A., Schmidt N.H., Parbrook P.J., Wilkinson A.J., Watson I.M. 'Electron backscatter diffraction and electron channeling contrast imaging of tilt and dislocations in nitride thin films'. Phys. Rev. B. 2007;75(8):085301

[23] Picard Y.N., Twigg M.E., Caldwell J.D., Eddy Jr C.R., Mastro M.A., Holm R.T. 'Resolving the Burgers vector for individual GaN dislocations by electron channeling contrast imaging'. Scripta Materialia. 2009;61(8):773-6

[24] Hite J.K., Mastro M.A., Eddy Jr C.R. 'Approach for dislocation free GaN epitaxy'. J. Crystal Growth. 2010;312(21):3143-6 
[25] Schulze A., Strakos L., Vystavel T., Loo R., Pacco A., Collaert N., Vandervorst W., Caymax M. 'Nondestructive characterization of extended crystalline defects in confined semiconductor device structures'. Nanoscale. 2018;10(15):7058-66

[26] Callahan P.G., Haidet B.B., Jung D., Seward G.G., Mukherjee K. 'Direct observation of recombinationenhanced dislocation glide in heteroepitaxial GaAs on silicon'. Phys. Rev. Mat. 2018;2(8):081601

[27] Carnevale S.D., Deitz J.I., Carlin J.A., Picard Y.N., De Graef M., Ringel S.A., Grassman T.J. 'Rapid misfit dislocation characterization in heteroepitaxial III-V/Si thin films by electron channeling contrast imaging'. App. Phys. Lett. 2014;104(23):232111

[28] Yaung K.N., Kirnstoetter S., Faucher J., Gerger A., Lochtefeld A., Barnett A., Lee M.L. 'Threading dislocation density characterization in III-V photovoltaic materials by electron channeling contrast imaging'. $J$. Crystal Growth. 2016;453:65-70

[29] Picard Y.N., Kamaladasa R., De Graef M., Nuhfer N.T., Mershon W.J., Owens T., Sedlacek L., Lopour F. 'Future prospects for defect and strain analysis in the SEM via electron channeling'. Microscopy Today. 2012;20(2):12-6

[30] Zaefferer S., Elhami N.N. 'Theory and application of electron channelling contrast imaging under controlled diffraction conditions'. Acta Materialia. 2014;75:20-50

[31]Mansour H., Guyon J., Crimp M.A., Gey N., Beausir B., Maloufi A.N. 'Accurate electron channeling contrast analysis of dislocations in fine grained bulk materials'. Scripta Materialia. 2014;84:11-4

[32] Picard Y.N., Liu M., Lammatao J., Kamaladasa R., De Graef M. 'Theory of dynamical electron channeling contrast images of near-surface crystal defects'. Ultramicroscopy. 2014;146:71-8

[33] Pascal E., Hourahine B., Naresh-Kumar G., Mingard K., Trager-Cowan C. 'Dislocation contrast in electron channelling contrast images as projections of strain-like components'. Materials Today: Proceedings.

2018;5(Issue):14652-61

[34] Crimp M.A., Simkin B.A., Ng B.C. 'Demonstration of the g. bxu= 0 edge dislocation invisibility criterion for electron channelling contrast imaging'. phil. mag. lett. 2001;81(12):833-7

[35] Naresh-Kumar G., Thomson D., Nouf-Allehiani M., Bruckbauer J., Edwards P.R., Hourahine B., Martin

R.W., Trager-Cowan C. 'Electron channelling contrast imaging for III-nitride thin film structures'. Materials

Science in Semiconductor Processing. 2016;47:44-50

[36] Schwartz A.J., Kumar M., Adams B.L., Field D.P. (eds.). 'Electron Backscatter Diffraction in Materials Science', New York: Springer 2009

[37] Wilkinson A.J., Moldovan G., Britton T.B., Bewick A., Clough R., Kirkland A.I. 'Direct detection of electron backscatter diffraction patterns'. Phys. Rev. Lett. 2013;111(6):065506

[38] Vespucci S., Winkelmann A., Naresh-Kumar G., Mingard K.P., Maneuski D., Edwards P.R., Day A.P., O'Shea V., Trager-Cowan C. 'Digital direct electron imaging of energy-filtered electron backscatter diffraction patterns'. Phys. Rev. B. 2015;92(20):205301

[39] Wilkinson A.J., Britton T.B. 'Strains, planes, and EBSD in materials science'. Materials Today.

2012;15(9):366-76

[40] Wilkinson A.J., Meaden G., Dingley D.J. 'High-resolution elastic strain measurement from electron backscatter diffraction patterns: new levels of sensitivity'. Ultramicroscopy. 2006;106(4-5):307-13

[41] Littlewood P.D., Britton T.B., Wilkinson A.J. 'Geometrically necessary dislocation density distributions in Ti-6Al-4V deformed in tension'. Acta Materialia. 2011;59(16):6489-500

[42] Vilalta-Clemente A., Naresh-Kumar G., Nouf-Allehiani M., Parbrook P.J., Le Boulbar E.D., Allsopp D., Shields P.A., Trager-Cowan C., Wilkinson A.J. 'High-Resolution Electron Backscatter Diffraction in III-Nitride Semiconductors'. Microscopy and Microanalysis. 2015;21(S3):2217-8

[43] Vaudin M.D., Gerbig Y.B., Stranick S.J., Cook R.F. 'Comparison of nanoscale measurements of strain and stress using electron back scattered diffraction and confocal Raman microscopy’. Appl. Phys. Lett.

2008;93(19): 193116

[44] Dingley D.J., Wilkinson A.J., Meaden G., Karamched P.S. 'Elastic strain tensor measurement using electron backscatter diffraction in the SEM'. J. electron microscopy. 2010; 59(S1):S155-63

[45] Villert S., Maurice C., Wyon C., Fortunier R. 'Accuracy assessment of elastic strain measurement by EBSD'. J. Microscopy. 2009;233(2):290-301

[46] Ishido T., Matsuo H., Katayama T., Ueda T., Inoue K., Ueda D. 'Depth profiles of strain in AlGaN/GaN heterostructures grown on Si characterized by electron backscatter diffraction technique'. IEICE Electronics Express. 2007;4(24):775-81

[47] Vilalta-Clemente A., Naresh-Kumar G., Nouf-Allehiani M., Gamarra P., di Forte-Poisson M.A., TragerCowan C., Wilkinson A.J. 'Cross-correlation based high resolution electron backscatter diffraction and electron channelling contrast imaging for strain mapping and dislocation distributions in InAlN thin films'. Acta Materialia. 2017;125:125-35

[48] Liu R., Schaller R., Chen C.Q., Bayram C. 'High Internal Quantum Efficiency Ultraviolet Emission from Phase-Transition Cubic GaN Integrated on Nanopatterned Si (100)'. ACS Photonics. 2018;5(3):955-63 
[49] Naresh-Kumar G., Vilalta-Clemente A., Jussila H., Winkelmann A., Nolze G., Vespucci S., Nagarajan S., Wilkinson A.J., Trager-Cowan C. 'Quantitative imaging of anti-phase domains by polarity sensitive orientation mapping using electron backscatter diffraction’. Scientific Reports. 2017;7(1):10916

[50] Winkelmann A., Nolze G., Himmerlich M., Lebedev V., Reichmann A. 'Point-Group Sensitive Orientation Mapping Using EBSD'. Proceedings of the 6th International Conference on Recrystallization and Grain Growth; Pittsburgh, USA, Jul 2016 . Springer, Cham. pp. 281-286.

[51] Momma K., Izumi F. 'VESTA 3 for three-dimensional visualization of crystal, volumetric and morphology data'. J. Appl. Cryst. 2011;44(6):1272-6

[52] Yacobi B.G., Holt D.B. 'Cathodoluminescence Microscopy of Inorganic Solids'. Plenum Press, 1990

[53] Chichibu S., Wada K., Nakamura S. 'Spatially resolved cathodoluminescence spectra of InGaN quantum wells'. Appl. Phys. Lett. 1997;71:2346

[54] Sugahara T., Sato H., Hao M., Naoi Y., Kurai S., Tottori S., Yamashita K., Nishino K., Romano L.T., Sakai S. 'Direct evidence that dislocations are non-radiative recombination centers in GaN'. Jpn. J. Appl. Phys. 1998;37:L398

[55] Ettenberg M., Kressel H., Gilbert S.L. 'Minority carrier diffusion length and recombination lifetime in GaAs:Ge prepared by liquid-phase epitaxy'. J. Appl. Phys. 1973;44:827

[56] Casey H.C., Miller B.I., Pinkas E. 'Variation of minority-carrier diffusion length with carrier concentration in GaAs liquidphase epitaxial layers'. J. Appl. Phys. 1973;44:1281

[57] Drouin D., Couture A.R., Joly D., Tastet X., Aimez V., Gauvin R. 'CASINO v2.42 - A fast and easy-to-use modelling tool for scanning electron microscopy and microanalysis users’. Scanning. 2007;29:92

[58] https://www.cstl.nist.gov/div837/837.02/epq/dtsa2/index.html

[59] Norman, C.E. 'Challenging the spatial resolution limits of CL and EBIC'. Solid State Phenom. 2001;19:7879

[60] Bruckbauer J., Edwards P.R, Wang T., Martin R.W. 'High resolution cathodoluminescence hyperspectral imaging of surface features in InGaN/GaN multiple quantum well structures'. Appl. Phys. Lett. 2011;98:141908 [61] Christen J., Grundmann M., Bimberg D. 'Scanning cathodoluminescence microscopy: A unique approach to atomic-scale characterization of heterointerfaces and imaging of semiconductor inhomogeneities'. J. Vac. Sci.

Technol. B. 1991;9:2358

[62] Martin R.W., Edwards P.R., O’Donnell K.P., Dawson M.D., Jeon C.-W., Liu C., Rice G.R., Watson I.M. 'Cathodoluminescence spectral mapping of III-nitride structures'. Phys. Stat. Sol. A. 2004;201:665

[63] Edwards P.R., Sleith D., Wark A.W., Martin R.W. 'Mapping localized surface plasmons within silver nanocubes using cathodoluminescence hyperspectral imaging'. J. Phys. Chem. C. 2011;115:14031

[64] Thonke K., Tischer I., Hocker M., Schirra M., Fujan K., Wiedenmann M., Schneider R., Frey M., Feneberg M. 'Nanoscale characterisation of semiconductors by cathodoluminescence'. IOP Conf. Series: Materials Science and Engineering. 2014;55:012018

[65] Naresh-Kumar G., Bruckbauer J., Edwards P.R., Kraeusel S., Hourahine B., Martin R.W., Kappers M.J., Moram M.J., Lovelock S., Oliver R.A., Humphreys C.J., Trager-Cowan C. 'Coincident electron channelling and cathodoluminescence studies of threading dislocations in GaN'. Microsc. Microanal. 2014;20:55

[66] Tischer I., Feneberg M., Schirra M., Yacoub H., Sauer R., Thonke K., Wunderer T., Scholz F., Dieterle L., Müller E., Gerthsen D. 'I $\mathrm{I}_{2}$ basal plane stacking fault in GaN: Origin of the $3.32 \mathrm{eV}$ luminescence band'. Phys. Rev. B. 2011;83:035314

[67] Lähnemann J., Jahn U., Brandt O., Flissikowski T., Dogan P., Grahn H.T. 'Luminescence associated with stacking faults in GaN'. J. Phys. D. 2014;47:423001

[68] Hsu P.S., Young E.C., Romanov A.E., Fujito K., DenBaars S.P., Nakamura S., Speck J.S. 'Misfit dislocation formation via pre-existing threading dislocation glide in semipolar heteroepitaxy'. Appl. Phys. Lett. 2011;99:081912

[69] Martineau P., Gaukroger M., Khan R., Evans D. 'Effect of steps on dislocations in CVD diamond grown on $\{001\}$ substrates'. Phys. Stat. Sol. C. 2009;6:1953

[70] Mukherjee K., Reilly C.H., Callahan P.G., Seward G.G.E. 'Recombination activity of threading dislocations in GaInP influenced by growth temperature'. J. Appl. Phys. 2018;123:165701

[71] Lee W., Chen J., Chen B., Chang J., Sekiguchi T. 'Cathodoluminescence study of dislocation-related luminescence from small-angle grain boundaries in multicrystalline silicon'. Appl. Phys. Lett. 2009;94:112103 [72] Knab M., Hocker M., Felser T., Tischer I., Wang J., Scholz F., Thonke K. 'EBIC investigations on polar and semipolar InGaN LED structures'. Phys. Stat. Sol. B. 2016;253:126

[73] Wallace M.J., Edwards P.R., Kappers M.J., Hopkins M.A., Oehler F., Sivaraya S., Allsopp D.W.E., Oliver R.A., Humphreys C.J., Martin R.W. 'Bias dependence and correlation of the cathodoluminescence and electron beam induced current from an InGaN/GaN light emitting diode'. J. Appl. Phys. 2014;116:033105

[74] Wallace M.J., Edwards P.R., Kappers M.J., Hopkins M.A., Oehler F., Sivaraya S., Oliver R.A., Humphreys C.J., Allsopp D.W.E., Martin R.W. 'Effect of the barrier growth mode on the luminescence and conductivity micron scale uniformity of InGaN light emitting diodes'. J. Appl. Phys. 2015;117:115705 
[75] Chen B., Chen J., Sekiguchi T., Ohyanagi T., Matsuhata H., Kinoshita A., Okumura H., Fabbri F. 'Electronbeam-induced current study of stacking faults and partial dislocations in Schottky diode'. Appl. Phys. Lett. 2008;93:033514

[76] Bandić Z.Z., Bridger P.M., Piquette E.C., McGill T.C. 'Electron diffusion length and lifetime in $p$-type GaN'Appl. Phys. Lett. 1998;73:3276

[77] Yakimov E.B. 'What is the real value of diffusion length in GaN?'. J. Alloys Compd. 2015;627:344

[78] Yakimov E.B., Polyakov A.Y., Smirnov N.B., Shchemerov I.V., Yang J., Ren F., Yang G., Kim J., Pearton S.J. 'Diffusion length of non-equilibrium minority charge carriers in $\beta-\mathrm{Ga}_{2} \mathrm{O}_{3}$ measured by electron beam induced current'. J. Appl. Phys. 2018;123:185704

[79] Yakimov E.B., Polyakov A.Y., Lee I.-H., Pearton S.J. 'Recombination properties of dislocations in GaN' J. Appl. Phys. 2018;123:161543

[80] Hamer P., Tweddle D., Martin T., Wilshaw P., Hiscock M., Lindsay J. 'EBIC-Enabled NanoManipulators Investigating Dislocations in mc-Solar Cells'. Microsc. Microanal. 2017;23:1426

[81] Moseley H.G.J. 'The high-frequency spectra of the elements'. Philosophical Magazine. 1914;27:703

[82] Friel J.J., Lyman C.E. 'X-ray Mapping in Electron-Beam Instruments'. Microsc. Microanal. 2006;12:2

[83] Edwards P.R., Martin R.W., O’Donnell K.P., Watson I.M. 'Simultaneous composition mapping and hyperspectral cathodoluminescence imaging of InGaN epilayers'. Phys. Stat. Sol. C. 2003;0:247

[84] Egerton R.F., Li P., Malac M. 'Radiation damage in the TEM and SEM'. Micron. 2004;35:399-409

[85] Banhart F. 'Irradiation effects in carbon nanostructures'. Rep. Prog. Phys. 1999;62:1181-1221

[86] Egerton R.F., McLeod R., Wang F., Malac M. 'Basic questions related to electron-induced sputtering in the TEM' Ultramicroscopy. 2010;110:991-997

[87] Cliff G., Lorimer G.W. 'The quantitative analysis of thin specimens'. J. Microscopy. 1975;103:203-207

[88]Hytch M.J., Snoeck E., Kilaaas R., 'Quantitative nanoscale characterisation by electron microscopy'.

Ultramicroscopy. 1998;74:131

[89] Perlin P., Suski T., Leszczyński M., Prystawko P., Świetlika T., Marona Ł., Wiśniewski P., Czernecki P., Nowak G., Weyher J.L., Kamler G., Borysiuk J., Litwin-Staszewska E., Dmowski L., Piotrzkowski R., Franssen G., Grzanka S., Grzegory I., Porowski S. 'Properties of InGaN blue laser diodes grown on bulk GaN substrates'.

J. Crystal Growth. 2005;281(1):107

[90] Dwilinski R., Doradzinski R., Garczynski J., Sierzputowski L.P., Puchalski A., Kanbara Y., Yagi K., Minakuchi H., Hayashi H., 'Excellent crystallinity of truly bulk ammonothermal GaN'. J. Crystal Growth. 2008;310(17):3911

[91] Frank F.C. 'Capillary equilibria of dislocated crystals'. Acta Crystallogr. 1951;4:497

[92] Oliver R.A., Kappers M.J., Sumner J., Datta R., Humphreys C.J. 'Highlighting threading dislocations in MOVPE-grown GaN using an in situ treatment with $\mathrm{SiH}_{4}$ and NH3'. J. Crystal Growth. 2006;289(2):506

[93] Oehler F., Zhu T., Rhode S. , Kappers M.J., Humphreys C.J., Oliver R.A. 'Surface morphology of homoepitaxial c-plane GaN: Hillocks and ridges'. J. Crystal Growth. 2013;38:12

[94] Das Bakshi S., Sumner J., Kappers M.J., Oliver R.A. 'The influence of coalescence time on unintentional doping in GaN/sapphire'. J. Crystal Growth. 2009;311(2):232-237

[95] Moram M.A., Oliver R.A.; Kappers M.J., Humphreys C.J. 'The Spatial Distribution of Threading Dislocations in Gallium Nitride Films'. Advanced Materials. 2009;21(38-39):3941

[96] Moram M.A., Johnston C.F., Kappers M.J., Humphreys C.J. 'Measuring dislocation densities in nonpolar aplane GaN films using atomic force microscopy'. J. Physics D - Applied Physics. 2010;43(5):055303

[97] Ketteniss N., Oliver R.A., McAleese C., Kappers M.J., Zhang Y., Humphreys C. J. 'The role of strain in controlling the surface morphology of $\mathrm{Al}_{\mathrm{x}} \mathrm{Ga}_{1-\mathrm{x}} \mathrm{N}$ following in situ treatment with $\mathrm{SiH}_{4}$ and $\mathrm{NH}_{3}$ '. Applied Surface Science. 2008;254(7):2124

[98] Rueden C.T., Schindelin J., Hiner M.C., DeZonia B.E., Walter A.E., Arena E.T., Eliceiri K.W. 'ImageJ2: ImageJ for the next generation of scientific image data.' BMC bioinformatics. 2017;18(1):529

[99] Naresh-Kumar G., Hourahine B., Edwards P.R., Day A.P., Winkelmann A., Wilkinson A.J., Parbrook P.J., England G., Trager-Cowan C. 'Rapid nondestructive analysis of threading dislocations in wurtzite materials using the scanning electron microscope'. Phys. Rev. Lett. 2012;108(13):135503

[100] Carnevale S.D., Deitz J.I., Carlin J.A., Picard Y.N., De Graef M., Ringel S.A., Grassman T.J. 'Rapid misfit dislocation characterization in heteroepitaxial III-V/Si thin films by electron channeling contrast imaging'. Appl. Phys. Lett. 2014;104(23):232111

[101] Ripley B.D. 'Modelling spatial patterns'. J. Royal Statistical Society. Series B (Methodological). 1977; 1:172-212

[102] Diggle P. 'A kernel method for smoothing point process data'. Applied statistics. 1985;1:138-47

[103] Besag J. 'Contribution to the discussion on Dr Ripley's paper'. JR Stat. Soc. 1977;39:193-5

[104] Gmeinwieser N., Schwarz U.T. 'Pattern formation and directional and spatial ordering of edge dislocations in bulk GaN: Microphotoluminescence spectra and continuum elastic calculations'. Phys. Rev. B.

2007;75(24):245213

[105] Moram M.A., Ghedia C.S., Rao D.V., Barnard J.S., Zhang Y., Kappers M.J., Humphreys C.J. 'On the origin of threading dislocations in GaN films'. J. appl. phys. 2009;106(7):073513 
[106] Moram M.A., Gabbai U.E., Sadler T.C., Kappers M.J., Oliver R.A. 'The use of spatial analysis techniques in defect and nanostructure studies'. J. electronic materials. 2010;39(6):656-62

[107] Trager-Cowan C., Naresh-Kumar G., Allehiani N., Kraeusel S., Hourahine B., Vespucci S., Thomson D., Bruckbauer J., Kusch G., Edwards P.R., Martin R.W., Mauder C., Day A. P., Winkelmann A., Vilalta-Clemente A., Wilkinson A. J., Parbrook P.J., Kappers M.J., Moram M.A., Oliver R.A., Humphreys C.J., Shields P., Le Boulbar E.D., Maneuski D., O'Shea V., Mingard K. P. 'Electron Channeling Contrast Imaging of Defects in IIINitride Semiconductors’ Microsc. Microanal. 2014;20:1024.

[108] Lozano J. G., Yang H., Guerrero-Lebrero M.P., D’Alfonso A.J., Yasuhara A., Okunishi E., Zhang S., Humphreys C.J., Allen L.J., Galindo P.L., Hirsch P.B.; Nellist P.D. 'Direct Observation of Depth-Dependent Atomic Displacements Associated with Dislocations in Gallium Nitride'. Phys. Rev. Lett. 2014;113(13):135503. [109] Richter E., Stoic T., Zeimer U., Netzel C., Weyers M., Tränkle G. 'Si Doping of GaN in Hydride VaporPhase Epitaxy'. J. Electronic Materials. 2013;42(5):820

[110] Zhu T., Oliver R.A. 'Unintentional doping in GaN'. Phys. Chem. Chem. Phys. 2012;14(27):9558-9573

[111] Gibart P. 'Metal organic vapour phase epitaxy of GaN and lateral overgrowth'. Rep. Prog. Phys. 2004;67(5):667-715

[112] Bennett S.E., Holec D., Kappers M.J., Humphreys C.J., Oliver R.A. 'Imaging dislocations in gallium nitride across broad areas using atomic force microscopy'. Review of Scientific Instruments. 2010;81(6):063701 [113] Sumner J., Oliver R.A., Kappers M.J., Humphreys C.J. 'Scanning capacitance microscopy studies of unintentional doping in epitaxial lateral overgrowth GaN'. J. Appl. Phys. 2009;106(10):104503

[114] Ilegems M., Montgomery H.C. 'Electrical properties of n-type vapor-grown gallium nitride'. J. Phys. Chem. Solids. 1973;34(5):885-895

[115] Hansen P.J., Strausser Y.E., Erickson A.N., Tarsa E.J., Kozodoy P., Brazel E.G., Ibbetson J.P., Mishra U. 'Scanning capacitance microscopy imaging of threading dislocations in GaN films grown on $\left(\begin{array}{llll}0 & 0 & 0 & 1\end{array}\right)$ sapphire by metalorganic chemical vapor deposition'. Appl. Phys. Lett. 1998;72(18):2247-2249

[116] Simpkins B.S., Yu E.T., Chowdhury U., Wong M.M., Zhu T.G., Yoo D.W., Dupuis R.D. 'Local conductivity and surface photovoltage variations due to magnesium segregation in p-type GaN'. J. Appl. Phys. 2004;95(11):6225-6231

[117] Rhode S., Horton M., Kappers M.J., Zhang S., Humphreys, C.J., Dusane R., Sahonta S.-L., Moram M.A. 'Mg Doping Affects Dislocation Core Structures in GaN'. Phys. Rev. Lett. 2013;111(2),025502

[118] Lee S.M., Belkhir M.A., Zhu X.Y., Lee Y.H., Hwang Y.G., Frauenheim T. 'Electronic structures of GaN edge dislocations'. Phys. Rev. B. 2000;61(23):16033

[119] Lymperakis L., Neugebauer J., Albrecht M., Remmele T., Strunk H.P. 'Strain Induced Deep Electronic States around Threading Dislocations in GaN'. Phys. Rev. Lett. 2004;93(19):196401

[120] Rhode S.L., Horton M.K., Fu W.Y., Sahonta S.-L., Kappers M.J., Pennycook T.J., Humphreys C.J., Dusane R.O., Moram M.A. 'Dislocation core structures in Si-doped GaN'. Appl. Phys. Lett.

2015:107(24):243104

[121] Rosner S.J., Carr E.C., Ludowise M.J., Girolami G., Erikson H. I. 'Correlation of cathodoluminescence inhomogeneity with microstructural defects in epitaxial GaN grown by metalorganic chemical-vapor deposition'. Appl. Phys. Lett. 1997;70:420

[122] Sugahara T., Sato H., Hao M., Naoi Y., Kurai S., Tottori S., Yamashita K., Nishino K., Romano L.T., Sakai S. 'Direct evidence that dislocations are non-radiative recombination centers in GaN'. Jpn. J. Appl. Phys. 1998;37:L398

[123] Hino T., Tomiya S., Miyajima T., Yanashima K., Hashimoto S., Ikeda M. 'Characterization of threading dislocations in GaN epitaxial layers'. Appl. Phys. Lett. 2000;76:3421

[124] Albrecht M., Weyher J.L., Lucznik B., Grzegory I., Porowski S. 'Nonradiative recombination at threading dislocations in n-type GaN: Studied by cathodoluminescence and defect selective etching'. Appl. Phys. Lett. 2008;92:231909

[125] Medvedev O., Vyvenko O., Ubyivovk E., Shapenkov S., Bondarenko A., Saring P., Seibt M. 'Intrinsic luminescence and core structure of freshly introduced a-screw dislocations in n-GaN'. J. Appl. Phys.

2018; 123:161427

[126] Brazel E.G., Chin M.A., Narayanamurti V. 'Direct observation of localized high current densities in GaN films'. Appl. Phys. Lett. 1999;74:2367

[127] Chen K.X., Dai Q., Lee W., Kim J.K., Schubert E.F., Grandusky J., Mendrick M., Li X., Smart J.A. 'Effect of dislocations on electrical and optical properties of n-type Al0.34Ga0.66N'. Appl. Phys. Lett. 2008;93:192108

[128] Kozodoy P., Ibbetson J.P., Marchand H., Fini P.T., Keller S., Speck J.S., DenBaars S.P., Mishra U.K. 'Electrical characterization of GaN p-n junctions with and without threading dislocations', Appl. Phys. Lett. 1998;73:975

[129] Stechmann G., Zaefferer S., Schwarz T., Konijnenberg P., Raabe D., Gretener C., Kranz L., Perrenoud J., Buecheler S., Nath Tiwari A. 'A correlative investigation of grain boundary crystallography and electronic properties in CdTe thin film solar cells'. Sol. Energy Mater. Sol. Cells. 2017;166:108 
[130] Nay Yaung K., Kirnstoetter S., Faucher J., Gerger A., Lochtefeld A., Barnett A., Larry Lee M. 'Threading dislocation density characterization in III-V photovoltaic materials by electron channelling contrast imaging'. $J$. Cryst. Growth. 2016;453:65

[131] Massabuau F.C-P., Chen P., Horton M.K., Rhode S.L., Ren C.X., O’Hanlon T.J., Kovacs A., Kappers M.J., Humphreys C.J., Dunin-Borkowski R.E., Oliver R.A. 'Carrier localization in the vicinity of dislocations in InGaN'. J. Appl. Phys. 2017;121:013104

[132] Prodan A., Marinković V., Gril R., Ramšak N., van Midden H.J.P., Boswell F.W., Bennett J.C. 'Scanning tunneling microscopy of defects in $\mathrm{NbSe}_{2}$ '. J. Vacuum Science \& Technology B. 2000;18(1):60-63

[133] Almadori Y., Bendiab N., Grévin B. 'Multimodal Kelvin Probe Force Microscopy Investigations of a Photovoltaic WSe $/ \mathrm{MoS}_{2}$ Type-II Interface'. ACS Appl. Mater. Interfaces. 2018;10:1363-1373

[134] Bampoulis P., Sotthewes K., Siekman M.H., Zandvliet H.J.W. 'Local Conduction in $\mathrm{Mo}_{x} \mathrm{~W}_{1-x} \mathrm{Se}_{2}$ : The Role of Stacking Faults, Defects, and Alloying'. ACS Appl. Mater. Interfaces. 2018;10:13218-13225

[135] Addou R., Wallace R.M. 'Surface Analysis of WSe2 Crystals: Spatial and Electronic Variability'. ACS Appl. Mater. Interfaces. 2016;8:26400-26406

[136] Huang Y.L., Ding Z., Zhang W., Chang Y.-H., Shi Y., Li L.-J., Song Z., Zheng Y.J., Chi D., Quek S.Y., Wee A.T.S. 'Gap States at Low-Angle Grain Boundaries in Monolayer Tungsten Diselenide'. Nano Lett. 2016;16:3682-3688

[137] Hite J.K., Twigg M.E., Tedesco J.L., Friedman A.L., Myers-Ward R.L., Eddy Jr C.R., Gaskill D.K. 'Epitaxial graphene nucleation on C-face silicon carbide'. Nano Lett. 2011;11(3):1190-4

[138] Bourrellier R., Meuret S., Tararan A., Stephan O., Kociak M., Tizei L.H.G., Zobelli A., Nano Lett. 2018;16:4317-4321

[139] Tran T.T., Bray K., Ford M.J., Toth M., Aharonovich I. 'Quantum emission from hexagonal boron nitride monolayers'. Nature Nano. 2016;11:37-41

[140] Gibb A.L., Alem N., Chen J-H., Erickson K.J., Ciston J., Gautam A., Linck M., Zettl A. 'Atomic resolution imaging of grain boundary defects in monolayer chemical vapor deposition-grown hexagonal boron nitride'. J. Am. Chem. Soc. 2013;135:6758-6761

[141] Alem N., Yazyev O.V., Kisielowski C., Denes P., Dahmen U., Hartel P., Haider M., Bischoff M., Jiang B., Louie S.G., Zettl A. 'Probing the Out-of-Plane Distortion of Single Point Defects in Atomically Thin Hexagonal Boron Nitride at the Picometer Scale'. Phys. Rev. Lett. 2011;106:126102

[142] Madsen J., Liu P., Kling J., Wagner J.B., Hansen T.W., Winther O., Schiotz J. ‘A Deep Learning Approach to Identify Local Structures in Atomic-Resolution Transmission Electron Microscopy Images'. Adv. Theory Simul. 2018;1:1800037

[143] Ziatdinov M., Dyck O., Maksov A., Li X., Sang X., Xiao K., Unocic R.R., Vasudevan R., Jesse S., Kalinin S.V. 'Deep Learning of Atomically Resolved Scanning Transmission Electron Microscopy Images: Chemical Identification and Tracking Local Transformations'. ACS Nano. 2017;11:12742-12752

[144] Griffiths J.T., Zhang S., Rouet-Leduc B., Fu W.Y., Bao A., Zhu D., Wallis, D.J., Howkins A., Boyd I., Stowe, D., Kappers M.J., Humphreys C.J., Oliver R.A. 'Nanocathodoluminescence Reveals Mitigation of the Stark Shift in InGaN Quantum Wells by Si Doping'. Nano Letters. 2015;15(11):7639

[145] Liu W., Carlin J.-F., Grandjean N., Deveaud B., Jacopin G. 'Exciton dynamics at a single dislocation in GaN probed by picosecond time-resolved cathodoluminescence'. Appl. Phys. Lett. 2016;109(04):042101

[146] Whiter R.A., Boughey C., Smith M., Kar-Narayan S. 'Mechanical Energy Harvesting Performance of Ferroelectric Polymer Nanowires Grown via Template-Wetting'. Energy Technol. 2018;6(5):928 